\title{
Newton Polygon and Gevrey Hierarchy in the Index Formulas for a Singular System of Ordinary Differential Equations
}

\author{
By
}

\author{
Masatake MiYAKE
}

(Nagoya University, Japan)

\begin{abstract}
We study a singular system of ordinary differential equations, $L u \equiv$ $\left\{z^{p+1} D I_{N}-A(z)\right\} u=0$, where $z \in C, p \geq 0, D=d / d z$ and $A(z)$ is an $N$ square matrix of holomorphic functions in a neighborhood of $z=0$. We call a matrix of operators $L \equiv z^{p+1} D I_{N}-A(z):=(p, A(z))$ a system, for short.

In this paper we introduce a notion of $T$-expansion of a matrix function $A(z)$, which gives a summation expression of $A(z)$ different from the usual Taylor expansion. The idea comes from the result by L. R. Volevič [Vol], where he studied a general matrix of partial differential operators $A\left(\partial_{x}\right)$ and he presented a way of finding out a leading part from the matrix operators which we call Volevič's lemma (cf. Section 3).

By using the $T$-expansion of $A(z)$, we obtain an algorithm of the reduction procedure of the system $L$ into a decomposition by irreducible subsystems (cf. Theorem $\mathrm{A}_{\delta}$ and (4.23) in Subsection 4.3). From this decomposition we can define the Newton polygon $\mathrm{N}(L)$ by taking the characteristic polynomial of each irreducible subsystem in Definitions 2.2 and 2.3. The importance of the Newton polygon $\mathrm{N}(L)$ will be shown by proving an index formula of the operator $L$ on a formal Gevrey space $\mathscr{G}^{s}(1 \leq s \leq \infty)$ in Theorem $\mathrm{C}^{(\infty)}$, which is obtained from the vertical coordinate of an associated vertex of $\mathrm{N}(L)$. This is an extension of J.-P. Ramis's results $[$ Ram1,2] for single operators. The index formula is proved by applying the index formula for general matrix of ordinary differential operators obtained in a joint paper with M. Yoshino [M-Y2]. Many other problems concerned with the study of the singular system $L=(p, A(z))$ are studied. For example, in Subsection 4.4 we give a structure of fundamental matrix solution of $L u=0$ in exact form. In other words, the reduction procedure into Hukuhara-Turrittin's canonical form is exactly shown. The reduction procedure seems to peel one piece of peel of an onion one piece.

Key Words and Phrases. Singular system, Newton polygon, Index formula, Formal Gevrey space, Equivalence transformation, Canonical form.

2010 Mathematics Subject Classification Numbers. Primary 34M25, Secondary $34 \mathrm{M} 35$.
\end{abstract}

\section{Contents}

1. Introduction

2. Statement of Main Results 178

2.1. Decomposability by irreducible subsystems 178 
2.2. Newton polygon and index formula on formal Gevrey space 180

3. Volevič's Lemma and $T$-Expansion 183

3.1. Volevič's lemma and its refinement 183

3.2. Reformulation of some definitions and $T$-expansion 185

4. Proof of Theorems $\mathrm{A}_{\tau}, \mathrm{A}_{\delta}$ and $\mathrm{B} \quad 188$

4.1. Preliminaries from $[\mathrm{M}-\mathrm{I}] \quad 188$

4.2. Blocked decomposability and proof of Theorems $\mathrm{A}_{\tau}$ and $\mathrm{A}_{\delta} \quad 190$

$\begin{array}{ll}\text { 4.3. Proof of Theorem B } & 197\end{array}$

4.4. Hukuhara-Turrittins's canonical form 202

5. Index Formula of System on Formal Gevrey Space 204

5.1. Short review of results in [M-Y2] 205

5.2. Proof of Theorems $\mathrm{C}^{(\infty)}$ and $\mathrm{C}^{(m)} \quad 207$

6. Equivalence Relation with Single Operators 215

6.1. Equivalence between system and single operator 215

6.2. Systemization of single operator 219

7. Some Numerical Experiments 225

7.1. Degenerate system $=$ Nilpotent principal matrix 225

7.2. Non degenerate system $=$ Non nilpotent principal matrix $\quad 227$

7.3. Full rank system when the principal matrix has only one eigenvalue

7.4. Full rank system when the principal matrix have distinct eigenvalues

\section{List of notations}

- $\boldsymbol{C}, \boldsymbol{R}, \boldsymbol{Q}, \boldsymbol{Z}, \boldsymbol{N}$; complex numbers or variable, real numbers or variable, rational numbers, integers, non negative integers.

- $\boldsymbol{C}[z], \boldsymbol{C}\{z\}, \boldsymbol{C}[[z]]$; polynomials, convergent series, formal power series over $\boldsymbol{C}$.

- $\mathscr{R}[z]:=\boldsymbol{C}[z] / \boldsymbol{C}[z]=\{f(z) / g(z) ; f(z), g(z)(\neq 0) \in \boldsymbol{C}[z]\} ; \quad$ rational functions,

$\mathscr{R}\{z\}:=\boldsymbol{C}\{z\} / \boldsymbol{C}\{z\} ;$ meromorphic functions at $z=0$,

$\mathscr{R}[[z]]:=\boldsymbol{C}[[z]] / \boldsymbol{C}[[z]]$; formal meromorphic functions at $z=0$.

- $M_{m \times n}(R)\left(M_{m}(R):=M_{m \times m}(R)\right) ; m \times n$-matrices with entries in a ring $R$.

- $G L_{m}(R)$; invertible matrices in $M_{m}(R)$.

- (matrix notation) For $A \in M_{m}(R)$ and $B \in M_{n}(R)$

$\operatorname{Diag}[A, B]=A \oplus B=\left[\begin{array}{cc}A & O \\ O & B\end{array}\right] \in M_{m+n}(R)$,

$\operatorname{Triang}[A, B]=\left[\begin{array}{cc}A & C \\ O & B\end{array}\right] \in M_{m+n}(R)$, (upper blocked triangular matrix). 
- (equivalence relation, transformation matrix) For two matrices $A, B \in$ $M_{N}(R)$ the equivalence relation is defined by an invertible matrix $P \in G L_{N}(R)$ by

$$
P: A \mapsto B \quad \stackrel{\text { def. }}{\Longleftrightarrow} \quad B=P^{-1} A P \equiv A_{P} .
$$

The matrix $P$ is called a transformation matrix from $A$ to $B$, and $B\left(=A_{P}\right)$ is called the reduced matrix. For two matrices $A, B \in$ $M_{N}(R)$, if there exists $P \in G L_{N}(R)$ such that $P: A \mapsto B$, then we say that they are equivalent which is denoted by

$$
A \sim B \quad \text { or } \quad A \sim_{R} B .
$$

- (formal Gevrey space) The formal $s$-Gevrey space $\mathscr{G}^{s}(1 \leq s \leq \infty)$, which is a subset of $\boldsymbol{C}[[z]]$, is defined that $\mathscr{G}^{\infty}=\boldsymbol{C}[[z]]$ and if $1 \leq s<\infty$,

$$
u(z)=\sum_{n=0}^{\infty} u_{n} z^{n} \in \mathscr{G}^{s} \quad \stackrel{\text { def. }}{\Longleftrightarrow}\left(\hat{\mathscr{B}}_{s} u\right)(\zeta):=\sum_{n=0}^{\infty} u_{n} \frac{\zeta^{n}}{(n !)^{s-1}} \in \boldsymbol{C}\{\zeta\} .
$$

It is often that $\hat{\mathscr{B}}_{s}$ is called the formal $s$-Borel transformation. The space $\mathscr{G}^{s}$ has the inductive limit topology of Banach spaces induced from that of $C\{\zeta\}$. That $\mathscr{G}^{1}=\boldsymbol{C}\{z\}$ is obvious.

- (order of zeros, pole order) For a function $a(z) \in \mathscr{R}[[z]], O(a)=r \in$ $\boldsymbol{Z} \cup\{\infty\}$ denotes the order of zeros of $a(z)$ at $z=0$, which means that, when $a(z) \not \equiv 0$ we have $O(a)=r \in \boldsymbol{Z}$ and $a(z)=\sum_{n=r}^{\infty} a_{n} z^{n}$ with $a_{r} \neq 0$. When $a(z) \equiv 0$, then $O(a)=\infty$. Therefore, $a(z) \in \mathscr{R}[[z]]$ has a pole singularity at $z=0$ when $O(a)<0$, and $-O(a)$ gives the pole order.

For a matrix function $A(z) \in M_{m \times n}(\mathscr{R}[[z]])$, the order of zeros $O(A) \in \boldsymbol{Z} \cup\{\infty\}$ is similarly defined, that is, $A(z)=\sum_{n=O(A)}^{\infty} A_{n} z^{n}$ $\left(A_{O(A)} \neq O\right)$ when $O(A) \in Z$.

- (Volevič's weight and V-numbers) For a matrix function $A(z)=\left(a_{i j}(z)\right) \in$ $M_{N}(\mathscr{R}[[z]])$, the Volevič weight $V(A) \in \boldsymbol{Q} \cup\{\infty\}$ is defined by

$$
V(A):=\min _{1 \leq n \leq N} \min _{1 \leq i_{1}<i_{2}<\cdots<i_{n} \leq N} \min _{\sigma \in S_{n}} \frac{1}{n} \sum_{k=1}^{n} r_{i_{k}, i_{\sigma(k)}}, \quad r_{i j}=O\left(a_{i j}\right),
$$

where $r<\infty$ and $r+\infty=\infty$ for $\forall r \in \boldsymbol{Q}$, and $\min _{1 \leq i_{1}<\cdots<i_{n} \leq N}$ means to take the minimum over all $n$-tuples $\left\{i_{k}\right\}_{k=1}^{n}$ as above, and $S_{n}$ denotes the permutation group of $\{1,2, \ldots, n\}$. Note that $V(A) \geq O(A)=$ $\min \left\{O\left(a_{i j}\right)\right\}$. For example, for

$$
A(z)=\left(\begin{array}{ll}
0 & 1 \\
0 & 0
\end{array}\right)
$$

we have $V(A)=\infty$ and $O(A)=0$. 
Let $q \in \boldsymbol{Q}$ be given. A numbers $T=\left\{t_{i}\right\}_{i=1}^{N} \in \boldsymbol{Q}$ is called the V-numbers (= Volevič numbers) associated with $q$ if the following inequalities hold,

$$
r_{i j} \geq t_{i}-t_{j}+q, \quad \forall i, j=1,2, \ldots, N .
$$

The existence of the $\mathrm{V}$-numbers associated with $V(A)$ is guaranteed by the Volevič lemma (cf. Subsection 3.1), but the uniqueness does not hold. In fact, they are translation free.

- (T-expansion, principal matrix function and principal matrix)

For $A(z)=\left(a_{i j}(z)\right) \in M_{N}(\mathscr{R}[[z]])$, we take $J \in N$ such that $V(A)=$ $m / J \in \boldsymbol{Z} / J \quad(m \in \boldsymbol{Z})$. Then we can take $T=\left\{t_{i}\right\} \in \boldsymbol{Z} / J$ for the Vnumbers, and put $t_{i}=s_{i} / J\left(s_{i} \in \boldsymbol{Z}\right)$. We expand each entry $a_{i j}(z)$ by

$$
a_{i j}(z)=\sum_{\ell=0}^{\infty} a_{i j}^{(\ell)} z^{\left(s_{i}-s_{j}+m+\ell\right) / J}, \quad a_{i j}^{(\ell)}=0 \text { if } \frac{s_{i}-s_{j}+m+\ell}{J} \notin \boldsymbol{Z} .
$$

By using this expansion, the matrix function $A(z)$ is expanded by

$$
A(z)=\sum_{\ell=0}^{\infty} A_{m+\ell}(z), \quad A_{m+\ell}(z)=\left(a_{i j}^{(\ell)} z^{\left(s_{i}-s_{j}+m+\ell\right) / J}\right) \in M_{N}(\mathscr{R}[z]),
$$

where $V\left(A_{m+\ell}\right)=(m+\ell) / J=V(A)+\ell / J$.

We call this expansion an expansion of $A(z)$ associated with $\mathrm{V}$-numbers $T$ or $T$-expansion for the simplicity. The usual Taylor or Laurent expansion is a $T$-expansion associated with $O(A)$ and $T=\left\{t_{i}=0\right\}_{i=1}^{N}$, which will be called a flat-expansion.

A matrix function $\AA_{T}(z):=A_{m}(z)=\left(a_{i j}^{(0)} z^{t_{i}-t_{j}+V(A)}\right) \in M_{N}(\mathscr{R}[z])$ and a constant matrix $\stackrel{\circ}{A}_{T}=\left(a_{i j}^{(0)}\right) \in M_{N}(C)$ are called the principal matrix function and the principal matrix associated with the $\mathrm{V}$-numbers $T=\left\{t_{i}\right\}$. These matrices depend on the choice of the V-numbers $T$, but their characteristic polynomials do not.

- (singular system of first kind in V-sense, full rank system of irregular singular type) A singular system of ordinary differential operators $L=$ $z^{p+1}(d / d z) I_{N}-A(z)$ is written by $L=(p, A(z))$.

A system $L=(p, A(z))$ is called a singular system of first kind in $\mathrm{V}$-sense if $V(A) \geq p$.

A system $L=(p, A(z))$ is called a full rank system of irregular singular type if $V(A)<p$ and that $\operatorname{det} \AA_{T} \neq 0$ or $O(\operatorname{det} A(z))=N V(A)$.

- (irreducible system)

A system $L=(p, A(x))$ is called an irreducible system if it is of first kind in V-sense or a full rank system of irregular singular type. 


\section{Introduction}

We study a matrix of first order linear ordinary differential operators $L(z, D)(D=d / d z)$ which is written in the form,

$$
\begin{aligned}
& L=(p, A(z)):=z^{p+1} D I_{N}-A(z), \quad p \in N, \\
& A(z)=\left(a_{i j}(z)\right)_{1 \leq i, j \leq N} \in M_{N}(\mathscr{R}[[z]]) .
\end{aligned}
$$

Hereafter we often omit to write $I_{N}$, the identity matrix. Throughout this paper, we call a matrix $L=(p, A(z))$ a system, since it is always related with a system of linear equations $L u=f$.

The most main interest in this paper is to show how we define the Newton polygon $\mathrm{N}(L)$ associated with the system $L$ from which the singular nature of the system $L$ is easily understood (cf. Definitions 2.2 and 2.3). For this purpose, as a typical problem of such kind, we shall prove an index formula of the operator $L=(p, A(z))$ in case $A(z) \in M_{N}(C\{z\})$ on formal Gevrey space $\mathscr{G}^{s}$, which is obtain from the vertical coordinate of an associated vertex of $\mathrm{N}(L)$ (cf. Theorems $\mathrm{C}^{(\infty)}$ and $\mathrm{C}^{(m)}$ ). This result is an extension of result by J.-P. Ramis in [Ram1,2] for single operators which will be explained below (cf. (1.9)).

The Newton polygon $\mathrm{N}(L)$ is defined after reducing the system $L$ into a decomposition by irreducible subsystems which is done by a formal meromorphic transformation (cf. Theorems $\mathrm{A}_{\tau}$ and $\mathrm{A}_{\delta}$ ). In doing so we have to arrow the pole singularity in the formal transformation matrix even if $A(z) \in C[[z]]$. This is the reason why we consider the system under $A(z) \in M_{N}(\mathscr{R}[[z]])$. The pole order in the formal transformation matrix to the irreducible decomposition is estimated in Theorem B.

After getting an irreducible decomposition of the system $L$ in Theorem $\mathrm{A}_{\delta}$, by continuing several already known equivalent transformations we get one step of reducing Hukuhara-Turritin's canonical form of $L=(p, A(z))$ (cf. [Was]). By repeating this reduction for each subsystem which appears in the procedure, we finally obtain Hukuhara-Turritin's canonical form by which the structure of the fundamental matrix solution of $L u=0$ is explicitly known (cf. Subsection 4.4). For example, the reason why and how we can choose a fractional power $z^{1 / r}(\exists r \in N)$ in the fundamental matrix solution will be clearly known.

It should be mentioned here that the most crucial and important device in this paper is that we introduced a new expression of a matrix function $A(z)$ different from the Taylor expansion, which we call a $T$-expansion of a matrix function $A(z)$. The idea of $T$-expansion was obtained from the observation by L. R. Volevič [Vol]. (cf. Volevič's lemma in Subsection 3.1 and Definition 3.7.) In fact, by taking $T$-expansion, we know an algorithm for the reduction procedure into the irreducible decomposition (cf. Proposition 4.3 and its proof). 
In the study of a singular system $L=(0, A(z))$ there is an idea of cyclic vector by $\mathrm{P}$. Deligne [Del] by which every system is reducible into a single operator. A study of the singular system from this point view was done by B. Malgrange [Mal] on the characterization of regular singular system. On this concern, we study the equivalence relation between systems and single operators in case of irregular singular system in Section 6.

In Section 7, we give some examples of numerical experiments for the reduction of systems of various type, which was done by Wolfram Mathematica 7.

This is the outline of problems studied in this paper.

For an easy understanding of our interest and results, let us give a short review of the results by J.-P. Ramis for a single operator, since the statements of results are very short, but are beautiful and impressible.

Without loss of generality, let a single operator $P \equiv P(z, D)$ be given by

$$
P(z, D)=\sum_{j=0}^{m} a_{j}(z)(z D)^{j}, \quad a_{j}(z) \in C\{z\}, a_{m}(z)=z^{p} .
$$

For an operator $a_{j}(z)(z D)^{j}$, we associate a figure $Q\left(j, O\left(a_{j}\right)\right) \subset \boldsymbol{R}^{2}$ by

$$
a_{j}(z)(z D)^{j} \leftrightarrow Q\left(j, O\left(a_{j}\right)\right)=\left\{(x, y) \in \boldsymbol{R}^{2} ; x \leq j, y \geq O\left(a_{j}\right)\right\}
$$

When $a_{j}(z) \equiv 0$, we understand that $Q\left(j, a_{j}\right)=\phi$. Then the Newton polygon $\mathrm{N}(P)$ associated with the operator $P$ is defined by

$$
\mathrm{N}(P):=\mathrm{Ch}\left(\bigcup_{j=0}^{m} Q\left(j, O\left(a_{j}\right)\right)\right),
$$

where $\mathrm{Ch}(S)$ denotes the convex hull of points in $S$.

By the assumption that $a_{m}(z)=z^{p}, \mathrm{~N}(P)$ has a fixed vertex $(m, p)$, and the figure of $\mathrm{N}(P)$ looks like




The Newton polygon $\mathrm{N}(P)$ is characterized by its vertexes, and we suppose that there are $k+1$-vertexes given by $\left\{\left(m_{i}, p_{i}\right)\right\}_{i=0}^{k}$ with

$$
m=m_{0}>m_{1}>\cdots>m_{k} \geq 0, \quad p=p_{0}>p_{1}>\cdots>p_{k} \geq 0 .
$$

It is known that the origin $z=0$ is a regular singular point if and only if $k=0$, that is, $O\left(a_{m}\right)=p \leq O\left(a_{j}\right)$ for $0 \leq \forall j \leq m-1$.

Here we recall that, by the definition, the origin $z=0$ is a regular singular point of $P$ if every solution $u(z)$ of $P u=0$ has at most polynomial growth of $|z|^{-1}$ as $z \rightarrow 0$ in every sector with a vertex $z=0$ of finite opening angle. A singular point which is not regular singular is called an irregular singular point. We already know that if $z=0$ is an irregular singular point, there is a solution $u(z)$ of $P u=0$ which has an exponential growth order in $|z|^{-1}$ as $z \rightarrow 0$ in some sector with a vertex $z=0$ of finite opening angle. The possible maximum of such exponential growth order between solutions is called the irregularity of the operator $P$ which we denote $\rho(P) \in \boldsymbol{R}_{>0}:=\{x \in \boldsymbol{R} ; x>0\}$. For the convenience sake the regular singular point case is understood as $\rho(P)=0$, and the irregularity is taken $\rho(P) \geq 0$.

Our interest is in the case of irregular singular point which means that $k \geq 1$ in (1.5). Let the slopes of sides of $\mathrm{N}(P)$ be $\left\{\rho_{i}\right\}_{i=0}^{k+1}$,

$$
\rho_{0}=\infty, \quad \rho_{i}=\frac{p_{i}-p_{i-1}}{m_{i}-m_{i-1}} \quad(1 \leq i \leq k), \quad \rho_{k+1}=0,
$$

where $\infty=\rho_{0}>\rho_{1}>\rho_{2}>\cdots \rho_{k}>\rho_{k+1}=0$. We define the numbers $\left\{s_{i}\right\}_{i=0}^{k}$ by

$$
s_{0}=1, \quad s_{i}=1+\frac{1}{\rho_{i}} \quad(1 \leq i \leq k), \quad s_{k+1}=\infty,
$$

where $1=s_{0}<s_{1}<s_{2}<\cdots<s_{k}<s_{k+1}=\infty$.

The above defined numbers have important informations on the singular nature of the operator $P$.

1. (The irregularity $\rho(P)=$ steepest slope $\left.\rho_{1}\right)$ It holds that $\rho(P)=\rho_{1}$. This means that for every solution of $P u(z)=0$ we have $|u(z)| \leq$ $C \exp \left[\delta|z|^{-\rho_{1}}\right]$ as $z \rightarrow 0$ in every sector $S$ with a vertex $z=0$ of finite opening angle, and there exists a solution for which $\rho_{1}$ is the best possible number.

2. $\quad$ (Maillet type theorem $=$ slowest slope $\rho_{k}$ ) The lowest positive slope $\rho_{k}$ relates with the order of divergence of formal solutions of $P u(z)=$ $f(z) \in \boldsymbol{C}\{z\}$. Precisely, if $u(z) \in \boldsymbol{C}[[z]]$ satisfies $P u \in \boldsymbol{C}\{z\}$, then it holds that $u(z) \in \mathscr{G}^{s_{k}}$, which is the best possible. Such a result is called a Maillet type theorem. 
In order to know the meaning of the intermediate slopes and vertexes, the following observation by J.-P. Ramis [Ram1,2] is excellent and beautiful. Let us consider the mapping for $1 \leq s \leq \infty$,

$$
P: \mathscr{G}^{s} \rightarrow \mathscr{G}^{s} .
$$

He proved that this is a Fredholm operator with an index $\chi\left(P ; \mathscr{G}^{s}\right):=$ $\left.\left.\operatorname{dim}_{C} \operatorname{Ker}\left(P: \mathscr{G}^{s} \rightarrow \mathscr{G}^{s}\right)\right)-\operatorname{dim}_{C} \operatorname{Coker}\left(P: \mathscr{G}^{s} \rightarrow \mathscr{G}^{s}\right)\right)$, which is a right continuous step function on $s \in[1, \infty]$ with $k$-discontinuous points $\left\{s_{i}\right\}_{i=1}^{k}$, given by

$$
\begin{aligned}
& \chi\left(P ; \mathscr{G}^{s}\right)= \begin{cases}-p_{0}, & \left(1=s_{0} \leq s<s_{1}\right), \\
-p_{i}, & \left(s_{i} \leq s<s_{i+1}, 1 \leq i \leq k-1\right), \\
-p_{k}, & \left(s_{k} \leq s \leq+\infty\right) .\end{cases} \\
& \chi\left(P, \mathcal{G}^{s}\right)
\end{aligned}
$$

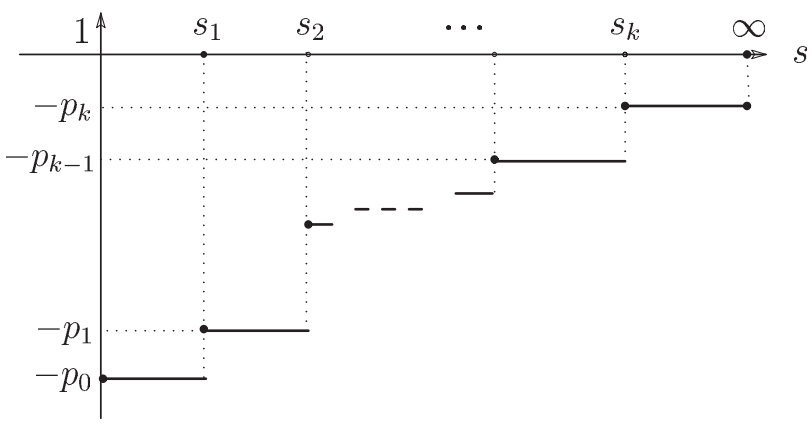

We call this phenomenon the Gevrey hierarchy in the index formulas which is seen in the title.

We have to remark the work by B. Malgrange [Mal] preceded J.-P. Ramis, in which he defined the notion of irregularity $i(P)$, which is different from $\rho(P)$, by

$$
i(P):=\operatorname{dim}_{C} \operatorname{Ker}(P ; \boldsymbol{C}[[z]] / \boldsymbol{C}\{z\} \rightarrow \boldsymbol{C}[[z]] / \boldsymbol{C}\{z\}) .
$$

Because of the surjectivity of the mapping $P: \boldsymbol{C}[[z]] / \boldsymbol{C}\{z\} \rightarrow \boldsymbol{C}[[z]] / \boldsymbol{C}\{z\}$, he actually showed

$$
i(P)=\chi(P ; \boldsymbol{C}[[z]] / \boldsymbol{C}\{z\})=\chi(P ; \boldsymbol{C}[[z]])-\chi(P ; \boldsymbol{C}\{z\})=p_{0}-p_{k} \geq 0 .
$$

Hence, the nullity of the irregularity $i(P)$ characterizes the regular singularity of $P$ at $z=0$, which means that $\mathrm{N}(P)$ has only one vertex $(m, p)$ as mentioned before.

Now we return to the study of the system $L=(p, A(z))$.

In case $L=(0, A(z))\left(A(z) \in M_{N}(\mathscr{R}\{z\})\right)$, the Newton polygon $\mathrm{N}(L)$ was defined by $\mathrm{B}$. Malgrange [Mal] after reducing $L$ into a single operator by 
Deligne's cyclic vector [Del]. Another definition is found in a book by P.-F. Hsieh and Y. Sibuya [Hs-Si] where the Newton polygon $\mathrm{N}(L)$ is defined by using the fundamental matrix solutions of $L u(z)=0$, which is equivalent with the definition by Malgrange. The information from the Newton polygon $\mathrm{N}(L)$ is not so much than those from the fundamental matrix solution. In fact, the Newton polygon $\mathrm{N}(L)$ gives us only the information of the leading part of determining factor, which concerns with the exponential growth in the fundamental matrix solution (cf. Subsection 4.4).

So the motivation of this study is that we would like to have a more direct and an elementary way from the system $L=(p, A(z))$ itself for the definition of the Newton polygon. Actually, this will be done by reducing the system $L$ into a decomposed form by irreducible subsystems, from which the Newton polygon is obtained by taking the characteristic polynomial for each irreducible subsystem (cf. Definitions 2.2 and 2.3).

As mentioned before, the reduction is done by taking $T$-expansion for a matrix function $A(z)$ which comes from the observation by L. R. Volevič [Vol].

We note that the first who used the idea by Volevič in the study of singular system of ordinary differential equations was K. Kitagawa [Kit] (cf. His idea comes from [Miy1] on the Cauchy-Kowalevski theorem for general system of p.d.e). In the paper, he characterized regular singular system for $L=(0, A(z))$ in case $A(z) \in M_{N}(\mathscr{R}\{z\})$. The Volevič idea was used when he take out a principal part from a matrix function $A(z)$. The obtained result was similar but different from Moser's characterization of regular singular system [Mos] (cf. [Lut] and [L-J] for the characterization of regular singular system).

In a joint paper with $\mathrm{K}$. Ichinobe [M-I], we extended the argument in [Kit] and obtained characterizations of the irregularity $\rho(L)$ for a system $L=$ $(p, A(z))\left(A(z) \in M_{N}(C\{z\})\right)$ in various form. Now we know that the paper [M-I] was devoted to the characterization of the steepest slope of the Newton polygon $\mathrm{N}(L)$, which gives a motivation of the study in this paper.

The idea of taking $T$-expansion of a matrix function $A(z)$, which is most important in this paper, comes from the device to kill the off diagonal block from the matrix $B(z)$ in Proposition 4.2 which was proved in [M-I, Prop. 4.1].

At the end of this introduction, we have to mention that the notion of Newton polygon is very important in the study of $k$-summability or the multisummability for divergent formal power series solutions, which was developed by J.-P. Ramis. But we do not treat this problem in this paper which will be done in a forth coming paper. (cf. [Ram3], [R-S], [Lod1,2], [Bal1,2], [Bra], etc.)

Acknowledgement. The author would like to express his thanks to the referee who kindly notify the references on the reduction problem for singular systems by M. A. Barkatou and E. Pflügel (cf. [Bar], $[\mathrm{B}-\mathrm{P}]$ and $[\mathrm{Pfl}]$ ). The 
works are devoted to establish an effective algorithm of computing the fundamental matrix solution of a singular system $x^{q+1} d Y / d x=A(x) Y$ with a formal power series $A(x)=\sum_{n=0}^{\infty} A_{n} x^{n}$, and of the reduction problem of the system. Their reductions are based on 1) Moser's criterion for the irreducibility of system which is stated in terms of $A_{0}$ and $A_{1}$ in the expansion of $A(x)$ (cf. [Mos]), and 2) Deligne's cyclic vector to obtain the Newton polygon or the Katz invariant which is the steepest slope of the Newton polygon (cf. [Del], [Mal] and [Hs-Si]). Such a viewpoint of their works is the great difference from the motivation of this paper.

\section{Statement of main results}

\subsection{Decomposability by irreducible subsystems}

The fundamental in this paper is that every singular system $L=(p, A(z))$ $\left(A(z) \in M_{N}(\mathscr{R}[[z]])\right)$ is reducible into a decomposed form by irreducible subsystems by a formal meromorphic transformation. Before we get a complete decomposition by irreducible subsystems, we reduce the system into a blocked triangular form. For the definitions of terminologies, see the List of Notations.

Theorem $\mathbf{A}_{\tau}$ (reduction into a blocked triangular form). A system $L=$ $(p, A(z))\left(A(z) \in M_{N}(\mathscr{R}[[z]])\right)$ is reducible into an upper blocked triangular form by a formal transformation matrix $P_{\tau}(z) \in G L_{N}(\mathscr{R}[[z]])$ of the following form; there is a decomposition $N=N_{1}+\cdots+N_{k}+N_{k+1}$ with $N_{i}>0(1 \leq i \leq k, k \geq 0)$ and $N_{k+1} \geq 0$ for which we have

$$
P_{\tau}(z): L \mapsto L_{P_{\tau}}:=P_{\tau}^{-1} L P_{\tau}=\left(p, \operatorname{Triang}\left[A_{1}(z), \ldots, A_{k}(z), A_{k+1}(z)\right]\right),
$$

where Triang $[\cdots]$ denotes an upper blocked triangular matrix, with the properties that the subsystems on the diagonal positions

$$
L_{i}=\left(p, A_{i}(z)\right), \quad 1 \leq i \leq k+1,
$$

are all irreducible such that $L_{i}$ is of full rank of irregular singular type for $1 \leq i \leq k$ and $L_{k+1}$ is of first kind in $V$-sense such that

$$
V\left(A_{1}\right)<V\left(A_{2}\right)<\cdots<V\left(A_{k}\right)<p \leq V\left(A_{k+1}\right) .
$$

Now the complete decomposability is stated as follows.

Theorem $\mathbf{A}_{\delta}$ (irreducible decomposition). Let a system $L=(p, A(z))$ be in Theorem $A_{\tau}$. Then $L$ is reducible by a transformation matrix $P_{\delta}(z) \in G L_{N}(\mathscr{R}[[z]])$ into a blocked diagonal form with the same irreducible diagonal subsystems in Theorem $A_{\tau}$.

$$
P_{\delta}(z): L \mapsto L_{P_{\delta}}=\bigoplus_{i=1}^{k+1} L_{i}(z, D), \quad L_{i}=\left(p, A_{i}(z)\right)
$$


Theorem $\mathrm{A}_{\delta}$ is obtained by killing the upper blocks of diagonal positions from $L_{P_{\tau}}$ in Theorem $\mathrm{A}_{\tau}$. The precise structure of the transformation matrices $P_{\tau}(z)$ and $P_{\delta}(z)$ will given by (4.24) and (4.25) in Subsection 4.3. By these expressions for $P_{\tau}(z)$ and $P_{\delta}(z)$, we can estimate the growth of the pole order or the defect of order of zeros of the matrices as follows.

Theorem $\mathbf{B}$ (growth estimate of pole order). For the matrices $A_{i}(z), P_{\tau}(z)$ and $P_{\delta}(z)$ in Theorems $A_{\tau}$ and $A_{\delta}$, we have the following estimates for $O\left(A_{i}\right)$, $O\left(P_{\tau}\right)$ and $O\left(P_{\delta}\right)$; Let $M_{1}=N$ and $M_{j}=N-\left(N_{1}+\cdots+N_{j-1}\right)$ for $2 \leq j \leq k$. Then

1) In case $N_{k+1}>0$, we have

$$
\left\{\begin{array}{l}
O\left(A_{i}\right) \geq-\prod_{j=1}^{i}\left(M_{j}-1\right)\{p-O(A)\}+p, \quad(1 \leq i \leq k) \\
\min \left\{O\left(P_{\tau}\right), O\left(P_{\delta}\right)\right\} \geq-\sum_{i=1}^{k} \prod_{j=1}^{i}\left(M_{j}-1\right)\{p-O(A)\}
\end{array}\right.
$$

and $O\left(A_{k+1}\right) \geq O\left(A_{k}\right)$.

2) In case $N_{k+1}=0$, the estimates for $\min \left\{O\left(P_{\tau}\right), O\left(P_{\delta}\right)\right\}$ and $O\left(A_{i}\right)$ $(1 \leq i \leq k-1)$ are the same with above, and $O\left(A_{k}\right) \geq O\left(A_{k-1}\right)$.

Remark 2.1. 1) In case $A(z) \in M_{N}(\mathscr{R}\{z\})$, the singular system $L=$ $(p, A(z))$ is regular singular at $z=0$ if and only if $k=0$ in Theorem $\mathrm{A}_{\tau}$, where the regular singularity of $L$ is defined by that every solution $u(z)$ of $L u(z)=0$ has at most polynomial growth in $|z|^{-1}$ as $z \rightarrow 0$ in any sector $S$ with a vertex at $z=0$ of finite opening angle. In this case a transformation matrix $P(z)$, which reduces the system $L$ into the first kind system in $\mathrm{V}$-sense, is taken in $G L_{N}(\boldsymbol{C}[z])$, i.e., polynomially invertible (cf. [Kit] and [M-I]).

2) In Theorem $\mathrm{B}$, the pole orders of the transformation matrices are estimated by knowing the decomposition $N=\sum_{i=1}^{k+1} N_{i}$ which is not known a priori. The worst growth estimate is taken when $N_{i}=1$ for each $N_{i}>0$ which gives

$$
\min \left\{O\left(P_{\tau}\right), O\left(P_{\delta}\right)\right\} \geq-\sum_{j=1}^{N-1} \frac{(N-1) !}{(N-1-j) !}\{p-O(A)\} \geq-e(N-1) !\{p-O(A)\}
$$

3) The formal fundamental matrix solution $W(z)$ of $L u(z)=0$ is given by

$$
W(z)=P(z) z^{G} \exp [\Lambda(z)], \quad \Lambda(z)=\operatorname{Diag}\left[\tilde{\Lambda}(z), O_{N_{k+1}}\right]
$$

where $P(z)$ is a matrix of formal power series of $z^{1 / r}(\exists r \in \boldsymbol{N}), G$ is a constant matrix and $\tilde{\Lambda}(z)$ is a diagonal matrix of entries of polynomials of $z^{-1 / r}$ without 
constant terms (cf. [Was]). The matrix $\Lambda(z)$ is called the determining factor. The problem to know how the number $r$ and $\Lambda(z)$ are determined seems to be interesting.

From the proof of Theorem B in Subsection 4.3 and the argument in Subsection 4.4, we know some informations on this problem as follows. Let $\AA_{i}$ be the principal matrix of $A_{i}(z)$ of $L_{i}=\left(p, A_{i}(z)\right) \quad(1 \leq i \leq k)$. In case when $A_{i}$ have distinct eigenvalues for all $1 \leq i \leq k$, the number $r$ is taken by the least common multiple of the denominators of $V\left(A_{i}\right)$ for $1 \leq i \leq k$, or in roughly, it is a divisor of the least common multiple of $N_{i}(1 \leq i \leq k)$. Therefore in the case when $N_{i}=1$ for $1 \leq i \leq k$, we can take $r=1$ (cf. Remark 4.9).

Let $\left\{\alpha_{\ell}^{(i)}\right\}_{\ell=1}^{N_{i}}$ be the eigenvalues of $A_{i}(1 \leq i \leq k)$ with counting the multiplicities. Then the leading term of each diagonal entry of $\tilde{\Lambda}(z)$ is given by

$$
\frac{\alpha_{\ell}^{(i)}}{q_{i}-p} z^{q_{i}-p}, \quad q_{i}=V\left(A_{i}\right), \quad 1 \leq \ell \leq N_{i}, 1 \leq i \leq k .
$$

The number $p-q_{1}=\rho(L)$ is the irregularity of $L$ characterized in [M-I] (cf. Definition 4.1).

\subsection{Newton polygon and index formula on formal Gevrey spaces}

For a polynomial $p(z, \zeta)=\sum_{j=0}^{m} a_{j}(z)(z \zeta)^{j}\left(a_{j}(z) \in \mathscr{R}[[z]], \zeta \in \boldsymbol{C}\right)$, we define the Newton polygon $\mathrm{N}(p)$ as in the case of $p(z, D)$ which was defined by (1.4).

We, first, define the Newton polygon for an irreducible system by taking the characteristic polynomial.

Definition 2.2 (Newton polygon 1). For an irreducible system $L=(p, A(z))$ $\left(A(z) \in M_{N}(\mathscr{R}[[z]])\right)$, we define the Newton polygon $\mathrm{N}(L)$ by

$$
\mathrm{N}(L):=\mathrm{N}(\operatorname{det} L(z, \zeta))
$$

The figure of the Newton polygon $\mathrm{N}(L)$, in this case, looks like



full rank system with $V(A)=q<p$

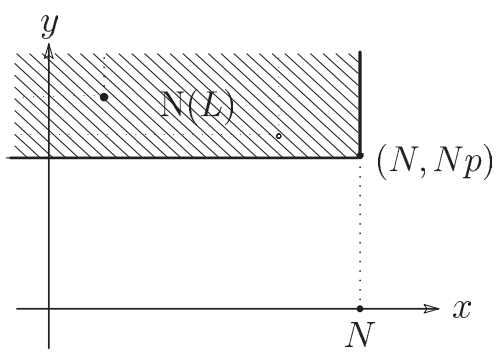

first kind system in $\mathrm{V}$-sense 
For a general system $L=(p, A(z))$ the Newton polygon is defined from the blocked decomposition in Theorem $\mathrm{A}_{\tau}$ or $\mathrm{A}_{\delta}$.

Definition 2.3 (Newton polygon 2). For a system $L=(p, A(z))(A(z) \in$ $\left.M_{N}(\mathscr{R}[[z]])\right)$, let $\bigoplus_{i=1}^{k+1} L_{i}\left(L_{i}=\left(p, A_{i}(z)\right)\right)$ be the irreducible decomposition in Theorem $\mathrm{A}_{\delta}$. Then the Newton polygon $\mathrm{N}(L)$ is defined by

$$
\mathrm{N}(L):=\mathrm{N}\left(L_{1}\right)+\cdots+\mathrm{N}\left(L_{k}\right)+\mathrm{N}\left(L_{k+1}\right),
$$

where the summation denotes the sum as point sets in a vector space $\boldsymbol{R}^{2}$.

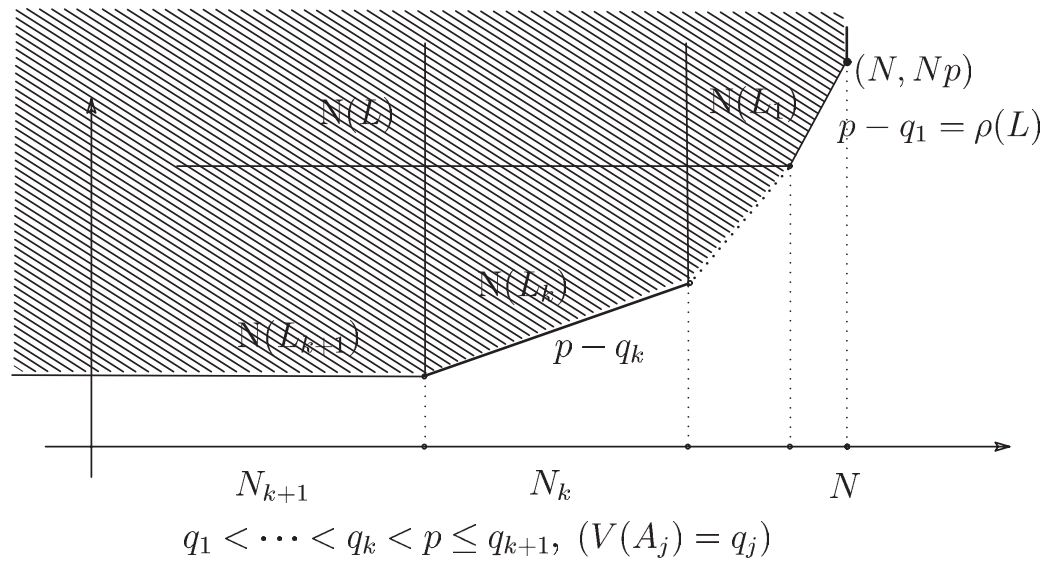

Remark 2.4. By the definition of the Newton polygon, each slope of $\mathrm{N}(L)$ corresponds with that of a subsystem in the irreducible decomposition. In Section 6, by employing Deligne's cyclic vector [Del] we shall give a decomposition by single operators each of which corresponds with the associated slope of $\mathrm{N}(L)$ (cf. Theorem 6.3). Moreover, a decomposition of a single operator $P(z, D)$ by sub-operators corresponding to the slopes of $\mathrm{N}(P)$ will be given (cf. Theorem 6.4).

In order to show the importance of the vertexes of $\mathrm{N}(L)$ we prove an index formula of the operator $L=(p, A(z))\left(A(z) \in M_{N}(\boldsymbol{C}\{z\})\right)$ on formal Gevrey space $\mathscr{G}^{s}(1 \leq s \leq \infty)$ as in the case of single operators. So let us consider the mapping,

$$
L:\left(\mathscr{G}^{S}\right)^{N} \rightarrow\left(\mathscr{G}^{S}\right)^{N}, \quad 1 \leq s \leq \infty .
$$

Then we can prove

Theorem $\mathbf{C}^{(\infty)}$ (Index formula). Let assume $A(z) \in M_{N}(C\{z\})$ for the system $L=(p, A(z))$. Then the mapping (2.8) have finite dimensional kernel 
and cokernel for all $1 \leq s \leq \infty$, and its index $\chi\left(L ; \mathscr{G}^{s}\right):=\operatorname{dim} \operatorname{Ker}\left(L ; \mathscr{G}^{s} \rightarrow \mathscr{G}^{s}\right)-$ $\operatorname{dim} \operatorname{Coker}\left(L: \mathscr{G}^{s} \rightarrow \mathscr{G}^{s}\right)$ is obtained by the same formula with (1.9) by using the vertical coordinates of the associated vertex of $\mathrm{N}(L)$.

Actually, the theorem is proved by truncating the reduction procedure to get the matrix $P_{\delta}(z) \in G L_{N}(\mathscr{R}[[z]])$ in Theorem $\mathrm{A}_{\delta}$ after a finite limited time as follows.

Let $P_{\delta}(z) \in G L_{N}(\mathscr{R}[[z]])$ be the transformation matrix in Theorem $\mathrm{A}_{\delta}$. For a fixed $m \in N$, we take $P_{\delta}^{(m)}(z) \in G L_{N}(\mathscr{R}[z])$ which is obtained by truncated the procedure to get $P_{\delta}(z)$ after a finite limited time such that for the reduced system

$$
P_{\delta}^{(m)}(z): L \mapsto L^{(m)}=\left(p,\left(A_{i j}^{(m)}(z)\right)_{i, j=1, \ldots, k+1}\right), \quad A_{i j}^{(m)}(z) \in M_{N_{i} \times N_{j}}(\mathscr{R}\{z\}),
$$

we have

$$
O\left(A_{i j}^{(m)}\right) \geq m \quad \text { for all } i \neq j .
$$

Here $m$ is taken so that for $1 \leq i \leq k A_{i i}^{(m)}(z)$ and $A_{i}(z)$ have a common Volevič weight, a common $\mathrm{V}$-numbers and a common principal matrix function and that $V\left(A_{k+1, k+1}^{(m)}\right) \geq p$.

Now our theorem is stated by

Theorem $\mathbf{C}^{(m)}$ (Index formula for truncated system). For the system $L=$ $(p, A(z))$ in the above, we have $\chi\left(L ; \mathscr{G}^{s}\right)=\chi\left(L^{(m)} ; \mathscr{G}^{s}\right)=\sum_{i=1}^{k+1} \chi\left(L_{i}^{(m)} ; \mathscr{G}^{s}\right)$ for all $1 \leq s \leq \infty$ if $m$ is taken so that;

1) When $P_{\delta}(z) \in G L_{N}(C[[z]])$ (without pole singularity), then

$$
m \geq p N .
$$

2) When $O\left(P_{\delta}\right)=-r<0$, then

$$
m+2 r \geq(p+2 r) N, \quad \text { i.e., } \quad m \geq p N+(N-1) 2 r .
$$

Note that $r$ is estimated from above by $r \leq e(N-1) ! \times p$ at worst. (cf. Remark 2.1-2)).

We give a simple example from which our results are easily known.

Example 2.5. Let us consider the following system,

$$
L_{q}=\left(2, A_{q}(z)\right)=z^{3} D-\left[\begin{array}{cc}
z^{q} & 1 \\
z^{3} & -z^{q}
\end{array}\right], \quad q \geq 1 .
$$

In case $q=1$ we have $V\left(A_{1}\right)=1$ and $L_{1}$ is a full rank system of irregular singular type, since $O\left(\operatorname{det} A_{1}(z)\right)=2$. In case $q \geq 2$ we have $V\left(A_{q}\right)=3 / 2$ and $L_{q}$ is a full rank system of irregular singular type, since $O\left(\operatorname{det} A_{q}(z)\right)=3=$ 
$2 \times(3 / 2)$. Since $\operatorname{det} L_{q}(z, \zeta)=z^{4}(z \zeta)^{2}-z^{2 q}-z^{3}$, the Newton polygon $\mathrm{N}\left(L_{1}\right)$ has two vertexes $\{(2,4),(0,2)\}$, and in case $q \geq 2 \mathrm{~N}\left(L_{q}\right)$ has two vertexes $\{(2,4),(0,3)\}$. Thus we see that the index formulas are given by

$$
\chi\left(L_{1} ; \mathscr{G}^{s}\right)=\left\{\begin{array}{ll}
-4, & 1 \leq s<2, \\
-2, & 2 \leq s \leq \infty
\end{array} . \quad \chi\left(L_{q} ; \mathscr{G}^{s}\right)=\left\{\begin{array}{ll}
-4, & 1 \leq s<3, \\
-3, & 3 \leq s \leq \infty .
\end{array} \quad(q \geq 2) .\right.\right.
$$

In this example the leading term of $A_{q}(z)$ by the Taylor expansion is

$$
A_{q}(0)=\left(\begin{array}{ll}
0 & 1 \\
0 & 0
\end{array}\right)
$$

which does not play any important role by itself, but the term

$$
\left(\begin{array}{cc}
0 & 1 \\
z^{3} & 0
\end{array}\right)
$$

for which the Volevič weight $3 / 2$, becomes a leading term when $q \geq 2$. This simple example may suggest that it is important to know how we look for the principal part from the coefficient matrix $A(z)$.

\section{Volevič's lemma and $T$-expansion}

\subsection{Volevič's lemma and its refinement}

The following two lemmas are called the Volevič lemma for the convenience.

Lemma 3.1 ([Vol], [Huf], [Miy1]). Let a matrix of numbers $\left(s_{i j}\right)_{1 \leq i, j \leq N}$ with $s_{i j} \in \boldsymbol{Z} \cup\{\infty\}$ (resp. $\left.\boldsymbol{Q} \cup\{\infty\}\right)$ satisfy

$$
s_{i i}=0, \quad \sum_{i=1}^{N} s_{i, \sigma(i)} \geq 0 \quad\left(\forall \sigma \in S_{N}\right) .
$$

Then there is a system of numbers $T=\left\{t_{i}\right\}_{i=1}^{N} \subset \boldsymbol{Z}$ (resp. Q) such that

$$
s_{i j} \geq t_{i}-t_{j}, \quad \forall i, j=1,2, \ldots, N .
$$

This lemma was given in $[\mathrm{Vol}]$ without proof and the existence proof was given by G. Hufford [Huf]. An actual choice of the numbers $T$ was shown in [Miy1] (cf. [M-I]). We repeat it here because it plays a key role in this paper. We, first, notice that the condition (3.1) implies the following inequalities.

$$
\sum_{k=1}^{n} s_{i_{k}, i_{\sigma(k)}} \geq 0, \quad \text { for all } 1 \leq i_{1}<\cdots<i_{n} \leq N, \sigma \in S_{n}, 1 \leq n \leq N .
$$


Then the numbers $T=\left\{t_{i}\right\}_{i=1}^{N}$ is chosen inductively as follows. First, a number $t_{1}$ is taken arbitrarily, since the numbers $T$ is translation free. Let suppose that $t_{k}(1 \leq k \leq i-1)$ are already determined. Then $t_{i}$ is chosen in the following non trivial interval.

$$
\begin{aligned}
& \max _{1 \leq p<i}\left\{t_{p}-s_{p, i_{1}}-s_{i_{1}, i_{2}}-\cdots-s_{i_{k}, i}\right\} \leq t_{i} \\
& \quad \leq \min _{1 \leq q<i}\left\{s_{i, j_{1}}+s_{j_{1}, j_{2}}+\cdots+s_{j_{\ell}, q}+t_{q}\right\},
\end{aligned}
$$

where $\left\{i_{1}, i_{2}, \ldots, i_{k}\right\},\left\{j_{1}, j_{2}, \ldots, j_{\ell}\right\} \subset\{i, i+1, \ldots, N\}$ are arbitrarily taken.

This shows that in Lemma 3.1 if $\left\{s_{i j}\right\} \subset \boldsymbol{Z} / J:=\{m / J ; m \in \boldsymbol{Z}\}$, then we can take the numbers $T=\left\{t_{j}\right\} \subset \boldsymbol{Z} / J$.

This lemma and the above observations imply the following,

Lemma 3.2. 1) For a matrix function $A(z)=\left(a_{i j}(z)\right) \in M_{N}(\mathscr{R}[[z]])$, let the Volevič weight be $V(A)=q$. Then we can find a numbers $T=\left\{t_{i}\right\} \subset \boldsymbol{Q}$ associated with $V(A)=q$ such that

$$
O\left(a_{i j}\right)=r_{i j} \geq t_{i}-t_{j}+q, \quad \forall i, j=1,2, \ldots, N .
$$

2) We take $J=\max \left\{n ; \min _{\sigma \in S_{n}} \sum_{k=1}^{n} r_{i_{k}, i_{\sigma(k)}}=n V(A)\right\}$. Then $V(A)=q \in$ $\boldsymbol{Z} / J$ and we can take the numbers $T=\left\{t_{i}\right\} \subset \boldsymbol{Z} / J$ in the statement 1).

3) If $V(A) \geq r \in \boldsymbol{Z} / J$, then there is a system of numbers $T=\left\{t_{i}\right\} \subset \boldsymbol{Z} / J$ such that $r_{i j} \geq t_{i}-t_{j}+r(\forall i, j=1,2, \ldots, N)$.

The statement 1) follows from Lemma 3.1 by putting $s_{i j}=r_{i j}-q$ for $i \neq j$ and $s_{i i}=\min \left\{r_{i i}-q, 0\right\}=0$. The statement 3$)$ is proved similarly. In the statement 2), it is obvious that $q \in \boldsymbol{Z} / J$ from the definition of $J$ and $q=V(A)$, which implies 2).

From Lemma 3.2-3), we know the reason why a singular system $L=$ $(p, A(z))$ with $V(A) \geq p$ is called of first kind system in $\mathrm{V}$-sense. In fact, there is a system of numbers $T=\left\{t_{i}\right\}_{i=1}^{N} \in \boldsymbol{Z}$ such that $O\left(a_{i j}\right) \geq t_{i}-t_{j}+p(\forall i, j)$. Let us define $z^{T}:=\operatorname{Diag}\left[z^{t_{1}}, \ldots, z^{t_{N}}\right]$. Then $z^{T}$ is a transformation matrix which reduces $L$ into a first kind system in usual sense;

$$
z^{T}: L \mapsto z^{-T} L z^{T}=z^{p}\{z D-B(z)\}, \quad B(z) \in M_{N}(C[[z]]) .
$$

We remark that K. Kitagawa [Kit] proved that in case $A(z) \in M_{N}(C\{z\})$ a system $L=(p, A(z))$ is regular singular at $z=0$ if and only if it is reducible into a first kind singular system in V-sense by a transformation matrix $P(z) \in$ $G L_{N}(\boldsymbol{C}[z])$. In $[\mathrm{M}-\mathrm{I}]$, we extended his method and obtained more precise classifications of the irregularity $\rho(L)$ for singular system $L=(p, A(z))$. These ideas are different from those developed by many other researchers, for example, J. Moser [Mos], D. Lutz [Lut] and W. Jurkat and D. Lutz [J-L]. 
Definition 3.3 (V-numbers, Span). For a given $q$, the numbers $T=\left\{t_{i}\right\}$ which satisfies (3.4) is called the Volevič numbers or V-numbers associated with q. By Lemma 3.2-3), if $r \leq V(A)$ we can find an associated $\mathrm{V}$-numbers $T$. The $\mathrm{V}$-numbers associated with $V(A)$ is called $\mathrm{V}$-numbers for short. The span $\sigma(T)$ of the $\mathrm{V}$-numbers $T$ is defined by

$$
\sigma(T)=\max _{i, j}\left|t_{i}-t_{j}\right|
$$

We have the following estimation for the span $\sigma(T)$.

Lemma 3.4 (Span condition). For $A(z) \in M_{N}(\mathscr{R}[[z]])$, we can find a $V$-numbers $T$ which satisfies

$$
\sigma(T) \leq(N-1)\{V(A)-O(A)\} .
$$

In case $A(z) \in M_{N}(C\{z\})\left(\subset M_{N}(C[[z]])\right)$, in this case we may assume $O(A)=0$, this estimate was proved in [M-I, Prop. 3.5]. The lemma follows from it by putting $B(z)=z^{-O(A)} A(z) \in M_{N}(C[[z]])(O(B)=0)$. Indeed, that $V(B)=V(A)-O(A)$ and that $B(z)$ and $A(z)$ have a common $\mathrm{V}$-numbers prove the assertion.

Hereafter, we take the V-numbers $T$ which satisfies the span condition (3.6) if there is no mentioning.

The span estimate (3.6) for $\sigma(T)$ will play a crucial role in the proof of Theorem $\mathrm{B}$ to estimate the pole order of matrices $A_{i}(z), P_{\tau}(z)$ and $P_{\delta}(z)$.

A principle for such estimation will be given by Proposition 3.8 in the next subsection.

\subsection{Reformulation of some definitions and $T$-expansion}

Our interest is in an irregular singular system, and we assume $q \leq$ $V(A)<p$. Let $T=\left\{t_{i}\right\}$ be the $\mathrm{V}$-numbers associated with $q$. Then each entry $a_{i j}(z)$ is written in the form

$$
a_{i j}(z)=\stackrel{\circ}{a}_{i j} z^{t_{i}-t_{j}+q}+o\left(|z|^{t_{i}-t_{j}+q}\right) \quad(z \rightarrow 0),\left(\stackrel{\circ}{a_{i j}}=0 \text { if } t_{i}-t_{j}+q \notin \boldsymbol{Z}\right) .
$$

Definition 3.5 (principal matrix, principal matrix function). We define matrices

$$
\stackrel{\circ}{A}_{T}:=\left(\stackrel{\circ}{a}_{i j}\right) \quad \text { and } \quad \stackrel{\circ}{A}_{T}(z):=\left(\stackrel{\circ}{a}_{i j} z^{t_{i}-t_{j}+q}\right)=z^{T} z^{q} \stackrel{\circ}{A}_{T} z^{-T} \in M_{N}(\mathscr{R}[z]),
$$

which we call the principal matrix and the principal matrix function associated with $q$ and $T$.

We note that these matrices depend on the choice of $\mathrm{V}$-numbers $T$, but their characteristic polynomials do not. It should be remarked that when 
$q=V(A), \stackrel{\circ}{A}_{T}$ can not be in the form of strictly upper or lower triangular matrix Triang $[0, \ldots, 0]$, which may be understood from the definition of $V(A)$. This shows that in the reduction procedure of the system $L=(p, A(z))$ by fixing the weight $q$ and $\mathrm{V}$-numbers $T$ if we meet a case when the principal matrix is a strictly triangular matrix, then the Volevič weight for the reduced system has become larger that $q$.

For example, consider a system

$$
L=(3, A(z)), \quad A(z)=\left(\begin{array}{cc}
z & 1 \\
-z^{2}+z^{3} & -z
\end{array}\right) .
$$

Then we have $V(A)=1, T=\left\{t_{1}=0, t_{2}=1\right\}$ and

$$
\stackrel{\circ}{A}_{T}=\left(\begin{array}{cc}
1 & 1 \\
-1 & -1
\end{array}\right)
$$

which is nilpotent. Then

$$
\left(\begin{array}{cc}
1 & 0 \\
-z & 1
\end{array}\right): L \mapsto L_{Q}=(3, B(z)), \quad B(z)=\left(\begin{array}{cc}
0 & 1 \\
z^{3}+z^{4} & 0
\end{array}\right) .
$$

For which we have

$$
\stackrel{\circ}{B}_{T}=\left(\begin{array}{cc}
0 & 1 \\
0 & 0
\end{array}\right)
$$

and $V(B)=3 / 2>V(A)$.

Definition 3.6 (full rank, non degenerate, degenerate). A singular system $L=(p, A(z))$ with $V(A)=q<p$ is called;

1) a full rank system of irregular singular type if $\operatorname{det} \stackrel{\circ}{A}_{T} \neq 0$.

This is equivalent with Definition 2.1. In this case, $J=N$ in Lemma $3.2-2)$.

2) a non degenerate system (of irregular singular type) if $\AA_{T}$ is not a nilpotent matrix.

3) a degenerate system if $\stackrel{\circ}{A}_{T}$ is a nilpotent matrix.

Recall that a system $L=(p, A(z))$ is said to be irreducible if it is a full rank system of irregular singular type or the first kind system in $\mathrm{V}$-sense.

In order to prove Theorems $\mathrm{A}_{\tau}$ and $\mathrm{A}_{\delta}$, it is convenient to take an expansion of $A(z) \in M_{N}(\mathscr{R}[[z]])$ which come from the Volevič weight $V(A)$ and the $\mathrm{V}$-numbers $T$ which we call a $T$-expansion. For a matrix function $A(z) \in \mathscr{R}[[z]]$, let assume $V(A)=q \in \boldsymbol{Z} / J \quad(q<p)$ and take the V-numbers $T=\left\{t_{i}\right\} \subset Z / J$. Let us write

$$
q=\frac{m}{J}, \quad t_{i}=\frac{s_{i}}{J} \quad\left(m,\left\{s_{i}\right\} \subset \boldsymbol{Z}\right) .
$$


We expand $a_{i j}(z) \in \mathscr{R}[[z]]$ by

$$
a_{i j}(z)=\sum_{\ell=0}^{\infty} a_{i j}^{(\ell)} z^{\left(s_{i}-s_{j}+m+\ell\right) / J}, \quad a_{i j}^{(\ell)}=0 \quad \text { if } \quad \frac{s_{i}-s_{j}+m+\ell}{J} \notin \boldsymbol{Z},
$$

where $a_{i j}^{(0)}=\stackrel{\circ}{a}_{i j}$. We take the following expansion for the matrix function $A(z) \in M_{N}(\mathscr{R}[[z]])$,

$$
A(z)=\sum_{\ell=0}^{\infty} A_{m+\ell}(z), \quad A_{m+\ell}(z)=\left(a_{i j}^{(\ell)} z^{\left(s_{i}-s_{j}+m+\ell\right) / J}\right)_{i, j=1, \ldots, N} \in M_{N}(\mathscr{R}[z]) .
$$

Definition 3.7 (T-expansion, flat-expansion). The expansion (3.9) is called the expansion by the $\mathrm{V}$-numbers $T$ or the $T$-expansion for short. The term $A_{m+\ell}(z)$ is a $T$-homogeneous part of degree $m+\ell$, actually $V\left(A_{m+\ell}\right)=$ $(m+\ell) / J$. The matrix function $A_{m}(z)\left(=A_{T}(z)\right)$ is the leading term in the $T$-expansion. The usual Taylor or Laurent expansion $A(z)=\sum_{n=O(A)}^{\infty} A_{n} z^{n}$ may be called the flat-expansion which means that we take the V-numbers $T=\left\{t_{i}=0\right\}_{i=1}^{N}$ associated with $O(A)$.

For a partial sum, we denote

$$
\sum_{\ell=k}^{\infty} A_{m+\ell}(z)=O(m+k), \quad V(O(m+k)) \geq \frac{m+k}{J} .
$$

By this notation we can write $A(z)=\sum_{\ell=0}^{k-1} A_{m+\ell}(z)+O(m+k)$.

The following pole estimates play important role in the proof of Theorem B.

Proposition 3.8. For a system $L=(p, A(z))\left(A(z) \in M_{N}(\mathscr{R}[[z]])\right)$, let assume $V(A)<p$ and $T=\left\{t_{i}=s_{i} / J\right\}_{i=1}^{N}$ be the associate $V$-numbers.

1) Let $P(z) \in G L_{N}(C[[z]])$ and take an equivalent transformation

$$
P(z): L=(p, A(z)) \mapsto L_{P}=(p, B(z)), \quad B(z)=P^{-1} A P-z^{p+1} P^{-1} P^{\prime}(z) .
$$

Then we have $O(B) \geq O(A)$.

2) Let $P(z) \in G L_{N}(\mathscr{R}[[z]])$ satisfy that $V(P)=0$ with $\stackrel{\circ}{P}_{T} \neq 0$ for the associated $V$-numbers $T$ which is common with $A(z)$. We take an equivalent transformation $P: L \mapsto L_{P}=(p, B(z))$ as above. Then we have

$$
\left\{\begin{aligned}
O(P) & \geq-\sigma(T) \geq-(N-1)\{V(A)-O(A)\}, \\
V(B) & \geq V(A), \\
O(B) & \geq-\sigma(T)+V(A) \geq-(N-1)\{V(A)-O(A)\}+V(A) \\
& =-(N-2)\{V(A)-O(A)\}+O(A) .
\end{aligned}\right.
$$


In case $N=2$, the last inequality shows that any defect of order of zeros does not occur even when the transformation matrix $P(z)$ has pole singularity.

Proof. The first statement is obvious.

2) The first inequality follows from the definition of the span $\sigma(T)$, by the assumption that $V(P)=0$. The second inequality follows from the definition of $T$-expansion. The third inequality follows from the following observation. Let $B(z)=\left(b_{i j}(z)\right)$. Then by the assumption that $V(A)<p$ we have

$$
O\left(b_{i j}\right) \geq t_{i}-t_{j}+V(A) \geq-\sigma(T)+V(A)
$$

implies that $O(B) \geq-\sigma(T)+V(A)$.

Remark 3.9. 1) The last inequality in (3.12) is not so trivial. In fact, an easy estimation implies $O\left(P^{-1} A P\right) \geq O\left(P^{-1}\right)+O(P)+O(A) \geq-3 \sigma(T)+V(A)$.

Example 3.10. Let a matrix function be

$$
A(z)=\left[\begin{array}{ccc}
0 & 1 & z \\
z^{2} & z & 0 \\
1 & 0 & z^{2}
\end{array}\right]
$$

Then $V(A)=1 / 2$. We take the V-numbers $T=\left\{t_{1}=1 / 2, t_{2}=3 / 2, t_{3}=0\right\}$. Then each $T$-homogeneous part in the $T$-expansion of $A(z)$ is given by

$$
\stackrel{\circ}{A}_{T}(z)=A_{1}(z)=\left[\begin{array}{ccc}
0 & 0 & z \\
0 & 0 & 0 \\
1 & 0 & 0
\end{array}\right], \quad A_{2}(z)=\left[\begin{array}{ccc}
0 & 1 & 0 \\
z^{2} & z & 0 \\
0 & 0 & 0
\end{array}\right], \quad A_{4}(z)=\left[\begin{array}{ccc}
0 & 0 & 0 \\
0 & 0 & 0 \\
0 & 0 & z^{2}
\end{array}\right] .
$$

\section{Proof of Theorems $\mathbf{A}_{\tau}, \mathbf{A}_{\delta}$ and $\mathbf{B}$}

\subsection{Preliminaries from $[\mathrm{M}-\mathrm{I}]$}

We, first, recall the reduction method into a non degenerate system given in $[\mathrm{M}-\mathrm{I}]$, which becomes the first step of the proof of Theorems $\mathrm{A}_{\tau}$ and $\mathrm{A}_{\delta}$.

Definition 4.1 (Irregularity for a system). For a system $L=(p, A(z))$ with $A(z) \in M_{N}(\mathscr{R}\{z\})$, we define a real number $\rho(L) \geq 0$, which we call the irregularity of $L$ by

$$
\rho(L):=\inf _{u, S}\left\{\rho>0 ;|u(z)| \leq C \exp \left[\delta|z|^{-\rho}\right], \forall z \in S, \exists C, \exists \delta>0\right\}
$$

where $\inf _{u, S}$ take over every solution $u(z)$ of $L u=0(z \neq 0)$, and every sector $S$ of finite opening angle with a vertex at $z=0$. The regular singular point is characterized by $\rho(L)=0$. 
In the previous paper $[\mathrm{M}-\mathrm{I}]$, we characterized the irregularity $\rho(L)$ in various different forms (cf. [M-I, Th.A]). The most fundamental characterization is stated as follows; "A system $L=(p, A(z))$ is irregular singular with $\rho(L)=\rho_{0}>0$ if and only if there exists a matrix $P(z) \in G L_{N}(\boldsymbol{C}[z])$ which reduces $L$ into

$$
P(z): L=(p, A(z)) \mapsto L_{P}=P^{-1} L P=(p, B(z))
$$

such that $L_{P}$ is a non degenerate system with $V(B)=p-\rho_{0}$."

We remark that the assumption that $A(z) \in M_{N}(\mathscr{R}\{z\})$ is meaningful in the growth estimate (4.1), but if we adopt the reducibility condition (4.2) for the definition of $\rho(L)$, we may assume $A(z) \in M_{N}(\mathscr{R}[[z]])$ without any difficulty. By this reason, in what follows, we use the notation $\rho(L)$ in the case of $A(z) \in M_{N}(\mathscr{R}[[z]])$, too.

For the proof of the above reducibility statement, the following proposition plays a crucial role which is the first step of the reduction into an irreducible decomposition.

Proposition 4.2 (cf. [M-I, Proposition 4.1]). For a singular system $L=$ $(p, A(z))$ with $A(z) \in M_{N}(\mathscr{R}[[z]])$, there exists a matrix $Q(z) \in G L_{N}(C[z])$ such that the reduced system

$$
Q(z): L \mapsto L_{Q}=Q^{-1} L Q=(p, B(z)),
$$

satisfies one of the following properties;

1) (regular singular case, $\rho(L)=0) L_{Q}$ is a first kind system in $V$-sense, i.e. $V(B) \geq p$.

2) (irregular singular case, $\rho(L)>0) L_{Q}$ is a non degenerate system of irregular singular type of $V(B)(<p)$ which satisfies the following properties; There are a V-numbers $S=\left\{s_{i}\right\}$ associated with $V(B)$ and a decomposition $N=N_{1}+M_{2}\left(1 \leq N_{1} \leq N\right)$ such that the principal matrix $\stackrel{\circ}{B}_{S}$ is written in an upper blocked triangular matrix associated with the decomposition of $N$,

$$
\stackrel{\circ}{B}_{S}=\operatorname{Triang}\left[\stackrel{\circ}{B}_{11}, \stackrel{\circ}{B}_{22}\right], \quad \stackrel{\circ}{B}_{i j} \text { are the blocked matrices, }
$$

with the following properties.

a) $\operatorname{det} \stackrel{\circ}{B}_{11} \neq 0$. This means that $\tilde{L}_{1}=\left(p, B^{11}(z)\right)$ is a full rank system of the irregularity $\rho\left(\tilde{L}_{1}\right)=p-V(B)(=\rho(L))$.

b) $\stackrel{\circ}{B}_{21}=O$ and $\stackrel{\circ}{B}_{22}=\operatorname{Triang}[0,0, \ldots, 0]$ is a strictly upper triangular matrix.

The above statements a) and b) indicate that $\stackrel{\circ}{B}_{11}$ becomes the principal matrix for the irreducible factor $L_{1}=\left(p, A_{1}(z)\right)$ in Theorem $\mathrm{A}_{\delta}$, which will be shown from the proof below. 
The detailed proof is given in $[\mathrm{M}-\mathrm{I}]$, but we show a key point of the proof since in the actual numerical examination in Section 7 we need it.

Let assume $V(A)<p$ and that $\operatorname{det} \stackrel{\circ}{A}_{T}=0$ for the principal matrix. In fact, if otherwise, $L=(p, A(z))$ is already an irreducible system. We rearrange the rows and columns of $A(z)$ so that $t_{1} \leq t_{2} \leq \cdots \leq t_{N}$ for the V-numbers $T=$ $\left\{t_{i}\right\}$. We choose a left null vector $\vec{\ell}(z)$ of $\stackrel{\circ}{A}_{T}(z)$, i.e. $\vec{\ell}(z) \stackrel{\circ}{A}(z)=0$, in the form $\vec{\ell}(z)=\left(\ell_{1}(z), \ldots, \ell_{k-1}(z), 1,0, \ldots, 0\right), \quad \ell_{i}(z)=\ell_{i} z^{t_{k}-t_{i}}, \quad \ell_{i}=0$ if $t_{k}-t_{i} \notin N$.

We define a matrix $Q(z) \in G L_{N}(C[z])$ so that

$$
Q^{-1}(z)=\left[\begin{array}{ccccccc}
1 & 0 & \ldots & 0 & 0 & \cdots & 0 \\
0 & 1 & \ldots & 0 & 0 & \cdots & 0 \\
\vdots & \vdots & \ddots & \ddots & \vdots & \vdots & \\
\ell_{1}(z) & \ldots & \ell_{k-1}(z) & 1 & 0 & \ldots & 0 \\
0 & 0 & \ldots & 0 & 1 & \ldots & 0 \\
\vdots & \vdots & \ldots & \vdots & \ddots & \ddots & \vdots \\
0 & 0 & \ldots & 0 & 0 & \cdots & 1
\end{array}\right],
$$

Let $L_{Q}=(p, C(z))$ be the reduced system by the matrix $Q(z)$, that is, $C(z)=Q^{-1} A Q-z^{p+1} Q^{-1} Q^{\prime}$. Then the following two cases occur.

(i) The case $V(C)>V(A)$. In this case, if $V(C)<p$ we repeat the above reduction for $L_{Q}$. Otherwise, we stop the reduction.

(ii) The case $V(C)=V(A)$. In this case, between the principal matrices we have $\stackrel{\circ}{C}_{T}=Q^{-1}(1) \AA_{T} Q(1)$. This shows that $k$-th row vector of $\stackrel{\circ}{C}_{T}$ vanishes. We send the $k$-th row and column to the $N$-th row and column, and we consider the subsystem $\left(p, C_{N-1}(z)\right)$ of size $N-1$ which is obtained by removing the $N$-th row and column from $L_{Q}$. For which we have $V\left(C_{N-1}\right)=V(A)$. Let the principal matrix be $\left(C_{N-1}^{\circ}\right)_{T^{\prime}}$ for $T^{\prime}=\left\{t_{i}\right\}_{i=1}^{N-1}$ to this subsystem.

If this principal matrix have no zero eigenvalues, then we stop the procedure since the system $L_{Q}$ is in the statement 2).

Otherwise, we continue the above operation to $\left(p, C_{N-1}(z)\right)$ by a transformation matrix $R_{N-1}(z)$ which is similarly defined with $Q(z)$. Then we take a transformation matrix $R(z)=R_{N-1}(z) \oplus 1$ for $L_{Q}$.

Following the situation (i) or (ii) for the reduced system $L_{Q R}$, we continue the operations, and we finally we meet a non degenerate system or a first kind system in V-sense.

\subsection{Blocked decomposability and proof of Theorems $\mathbf{A}_{\tau}$ and $\mathbf{A}_{\delta}$}

We start from the non degenerate system of irregular singular type $L_{Q}=(p, B(z))$ in Proposition 4.2-2). Let $T=\left\{t_{i}\right\}$ be the V-numbers of 
$B(z)$ associated with $V(B)=q(=p-\rho(L))$. By a technical reason, we rewrite the V-numbers $S$ by $T$ in Proposition 4.2-2).

We note that by the form of $B(z)$ we know that $V(B) \in Z / N_{1}$ (cf. Lemma 3.2-2)), and we put $q=m / N_{1}$ and $t_{i}=s_{i} / N_{1}$. We express $B(z)$ by $T$-expansion. This means that by the expression

$$
b_{i j}(z)=\sum_{\ell=0}^{\infty} b_{i j}^{(\ell)} z^{\left(s_{i}-s_{j}+m+\ell\right) / N_{1}}, \quad b_{i j}^{(\ell)}=0 \text { if } \frac{s_{i}-s_{j}+m+\ell}{N_{1}} \notin \boldsymbol{Z} .
$$

the $T$-expansion of $B(z)$ is given by

$$
\begin{aligned}
& B(z)=\sum_{\ell=0}^{\infty} B_{m+\ell}(z), \quad B_{m+\ell}(z)=\left(b_{i j}^{(\ell)} z^{\left(s_{i}-s_{j}+m+\ell\right) / N_{1}}\right) \in M_{N}(\mathscr{R}[z]), \\
& V\left(B_{m+\ell}\right)=\frac{m+\ell}{N_{1}} .
\end{aligned}
$$

By the assumption for the system $L_{Q}=(p, B(z))$, the principal matrix function $B_{m}(z)=\stackrel{\circ}{B}_{T}(z)$ has the following decomposition which comes from $N=N_{1}+M_{2}$,

$$
B_{m}(z)=\stackrel{\circ}{B}_{T}(z)=\left[\begin{array}{cc}
B_{m}^{11}(z) & B_{m}^{12}(z) \\
O & B_{m}^{22}(z)
\end{array}\right]
$$

where

$$
\operatorname{det} B_{m}^{11}(z)=c z^{q N_{1}} \neq 0, \quad B_{m}^{22}(z)=\left[\begin{array}{cccc}
0 & * & \cdots & * \\
0 & 0 & \cdots & * \\
\vdots & \ddots & \ddots & \vdots \\
0 & \cdots & 0 & 0
\end{array}\right] \in M_{M_{2}}(\mathscr{R}[z]) .
$$

Hereafter, matrix functions are written in the blocked form $X(z)=$ $\left(X^{i j}(z)\right)_{i, j=1}^{2} \in M_{N}(\mathscr{R}[[z]])$ corresponding to the above decomposition of $N$. Moreover, we assume that every matrix $X(z)$ has the common V-numbers $T$ with $B(z)=\left(B^{i j}(z)\right)$.

In order to reduce the system $L_{Q}=(p, B(z))$ into a blocked triangular form, we have to kill $(2,1)$ block $B^{21}(z)$ of $B(z)=\left(B^{i j}(z)\right)$.

Proposition 4.3 (blocked triangularization). Let $L_{Q}=(p, B(z))$ be as above. Then there is a unique matrix function $R(z) \in G L_{N}(\mathscr{R}[[z]])$ with $V$-numbers $T$ of $V(R)=0$ of the form

$$
R(z)=\left[\begin{array}{cc}
I_{N_{1}} & O \\
R^{21}(z) & I_{M_{2}}
\end{array}\right], \quad R_{0}(z)=I_{N} \text { (principal matrix function), }
$$


which brings

$$
R(z): L_{Q}=(p, B(z)) \mapsto L_{Q R}=(p, C(z)), \quad C^{21}(z)=O, V(C)=V(B),
$$

with $C(z)=\left(C^{i j}(z)\right)_{i, j=1}^{2}$ which satisfies

$$
C^{21}(z)=O, \quad V(C)=V\left(C^{11}\right)=V(B), \quad \stackrel{\circ}{C}_{T}(z)=\stackrel{\circ}{B}_{T}(z) .
$$

This means that $\left(p, C^{11}(z)\right)$ is a full rank system of irregular singular type which is nothing but the irreducible subsystem $L_{1}=\left(p, A_{1}(z)\right)$ in Theorems $A_{\tau}$ and $A_{\delta}$.

The orders of zeros of $R(z)$ and $C(z)$ are estimated by

$$
\left\{\begin{aligned}
O(R) & =O\left(R^{-1}\right) \geq-(N-1)(V(B)-O(B))>-(N-1)(p-O(A)) \\
O(C) & \geq-(N-1)(V(B)-O(B))+V(B) \\
& =-(N-2)(V(B)-O(B))+O(B)
\end{aligned}\right.
$$

where we used that $O(B) \geq O(A)$ since $Q(z) \in G L_{N}(C[z])$, and that $p>V(B) \geq$ $V(A)$ since $L$ is assumed to be of irregular singular type.

For the last estimates (4.8), see (3.12) in Proposition 3.8, which comes from the span estimate $\sigma(T) \leq(N-1)(V(B)-O(B))$.

A blocked decomposition is obtained immediately from the above proposition as follows.

Proposition 4.4 (blocked decomposition). Let $L_{Q R}=(p, C(z))$ be as in the above proposition. Then there is a unique matrix function $S(z) \in G L_{N}(\mathscr{R}[[z]])$ of $V(S)=0$ of the form

$$
\begin{aligned}
& S(z)=\left[\begin{array}{cc}
I_{N_{1}} & S^{12}(z) \\
O & I_{M_{2}}
\end{array}\right], \\
& S_{0}(z)=\left[\begin{array}{cc}
I_{N_{1}} & S_{0}^{12}(z) \\
O & I_{M_{2}}
\end{array}\right] \text { (principal matrix function), }
\end{aligned}
$$

which brings

$$
S(z): L_{Q R}=(p, C(z)) \mapsto L_{Q R S}=(p, D(z)), \quad D(z)=C^{11}(z) \oplus C^{22}(z) .
$$

The order of zeros $O(R S)$ is estimated from below by the same estimate for $O(R)$ in (4.8). Indeed, $R(z)$ and $S(z)$ we have $V(R)=V(R S)=0$ and they have a common $V$-numbers $T$.

Theorems $\mathrm{A}_{\tau}$ and $\mathrm{A}_{\delta}$ are immediate consequences from these propositions as follows.

Proof of Theorems $A_{\tau}$ and $A_{\delta}$. If $L=(p, A(z))$ is of regular singular type, the theorems are already proved in Proposition 4.2-1), i.e., the case $k=0$ in the theorems. If $L$ is of irregular singular type, by putting $A_{1}(z)=C^{11}(z)$ in 
the above propositions, $L_{1}=\left(p, A_{1}(z)\right)$ is the desired full rank subsystem of irregular singular type. By repeated use of the above operations for the subsystem $\left(p, C^{22}(z)\right)$ and so on, we finally obtain the desired blocked triangularization and the blocked decomposition by the irreducible subsystems in the theorems.

Precise structure of the transformation matrices $P_{\tau}(z)$ and $P_{\delta}(z)$ are given by (4.24) and (4.25) in the next subsection.

Now let us prove Propositions 4.3 and 4.4 which are obtained by the same argument given in the book [Was, Sec. 12], but the notion of $T$-expansion is essential.

Proof of Proposition 4.3. Suppose that the system $L_{Q}=(p, B(z))$ is reduced into a blocked triangular system $L_{Q R}=(p, C(z))\left(C^{21}(z)=O\right)$ by a matrix $R(z) \in G L_{N}(\mathscr{R}[[z]])$ given by (4.5). This means that $R^{-1}(z) B(z) R(z)-$ ${ }_{z^{p+1}} R^{-1}(z) d R(z) / d z=\left(C^{i j}(z)\right)\left(C^{21}(z)=O\right)$, that is,

$$
\begin{gathered}
{\left[\begin{array}{ll}
B^{11}(z) & B^{12}(z) \\
B^{21}(z) & B^{22}(z)
\end{array}\right]\left[\begin{array}{cc}
I_{N_{1}} & O \\
R^{21}(z) & I_{M_{2}}
\end{array}\right]-z^{p+1}\left[\begin{array}{cc}
O & O \\
R^{21}(z)^{\prime} & O
\end{array}\right]} \\
\quad=\left[\begin{array}{cc}
I_{N_{1}} & O \\
R^{21}(z) & I_{M_{2}}
\end{array}\right]\left[\begin{array}{cc}
C^{11}(z) & C^{12}(z) \\
O & C^{22}(z)
\end{array}\right] .
\end{gathered}
$$

This implies

$$
\begin{aligned}
& C^{11}(z)=B^{11}(z)+B^{12}(z) R^{21}(z), \quad C^{12}(z)=B^{12}(z), \\
& R^{21}(z) C^{11}(z)=B^{21}(z)+B^{22}(z) R^{21}(z)-z^{p+1} \frac{d}{d z} R^{21}(z), \\
& C^{22}(z)+R^{21}(z) C^{12}(z)=B^{22}(z) .
\end{aligned}
$$

By substituting the expression for $C^{11}(z)$ which is obtained from the first relation into the third relation, we get an equation for $R^{21}(z)$ as follows.

$$
\begin{aligned}
& B^{22}(z) R^{21}(z)-R^{21}(z) B^{11}(z) \\
& \quad=-B^{21}(z)+R^{21}(z) B^{12}(z) R^{21}(z)+z^{p+1} \frac{d}{d z} R^{21}(z) .
\end{aligned}
$$

Here we recall the $T$-expansion for the matrix functions,

$$
\left\{\begin{array}{l}
B(z)=\sum_{\ell=0}^{\infty} B_{m+\ell}(z), \quad B_{m+\ell}(z)=\left(B_{m+\ell}^{i j}(z)\right), \quad B_{m}^{21}(z)=O, \\
R(z)=\sum_{\ell=0}^{\infty} R_{\ell}(z), \quad R_{0}(z)=I_{N}, \quad R_{\ell}(z)=\left[\begin{array}{cc}
O & O \\
R_{\ell}^{21}(z) & O
\end{array}\right](\ell \geq 1) .
\end{array}\right.
$$


We note that an inequality $p N_{1}>m$ holds since $V(B)=m / N_{1}<p$. Hence by operating $z^{p+1} d / d z$ to $R_{k}(z)$ of $V\left(R_{k}\right)=k / N_{1}$, we have $V\left(z^{p+1}(d / d z) R_{k}\right)=$ $\left(k+p N_{1}\right) / N_{1}>(k+m) / N_{1}$.

Now we express the equation (4.11) for each homogeneous part in the $T$-expansion. The homogeneous part of order $m+1$ or the term of the Volevic weight $(m+1) / N_{1}$ is given by

$$
B_{m}^{22}(z) R_{1}^{21}(z)-R_{1}^{21}(z) B_{m}^{11}(z)=-B_{m+1}^{21}(z) .
$$

This is a linear equation for $R_{1}^{21}(z)$. The homogeneous part of order $m+\ell$ or the term of the Volevič weight $(m+\ell) / N_{1}$ is given by

$$
\begin{aligned}
B_{m}^{22}(z) & R_{\ell}^{21}(z)-R_{\ell}^{21}(z) B_{m}^{11}(z) \\
= & -B_{m+\ell}^{21}(z)+\sum_{a+b+c=\ell ; a, c \geq 1 ; b \geq 0} R_{a}^{21}(z) B_{m+b}^{12}(z) R_{c}^{21}(z) \\
& +z^{p+1} \frac{d}{d z} R_{\ell+m-p N_{1}}^{21}(z),
\end{aligned}
$$

where $m-p N_{1}<0$. This is a linear equation for $R_{\ell}^{21}(z)$, since on the right hand side the terms $R_{k}^{21}(z)$ with $k<\ell$ appear which are regarded as known.

Since $B_{m}^{22}(z)$ is a nilpotent matrix and $\operatorname{det}\left(B_{m}^{11}(z)\right) \neq 0$, we see that these linear equations are uniquely solvable in $M_{M_{2} \times N_{1}}(\mathscr{R}[z])$ from the following lemma.

Lemma 4.5 ([Was, Theorem 4.1]). Let $A \in M_{m}(\boldsymbol{C})$ and $B \in M_{n}(\boldsymbol{C})$ be given matrices. For a given matrix $Y \in M_{m \times n}(\boldsymbol{C})$ let us consider the following equation for $X \in M_{m \times n}(\boldsymbol{C})$,

$$
A X-X B=Y .
$$

Then if $A$ and $B$ have disjoint eigenvalues, the above equation is uniquely solvable.

Proof of Proposition 4.4. Let $L_{Q R}=(p, C(z))$ be the system obtained in Proposition 4.3. By a matrix function

$$
S(z)=\left[\begin{array}{cc}
I_{N_{1}} & S^{12}(z) \\
O & I_{M_{2}}
\end{array}\right]
$$

which satisfies the condition (4.9), we suppose that $L_{Q R}$ is decomposed into $L_{Q R S}=\left(p, C^{11}(z) \oplus C^{22}(z)\right)$. Then, as in the previous proof, it is examined that $S^{12}(z)$ should satisfy the following equation which is linear but not nonlinear.

$$
C^{11}(z) S^{12}(z)-S^{12}(z) C^{22}(z)=-C^{12}(z)+z^{p+1} \frac{d}{d z} S^{12}(z)
$$


The difference between this equation and (4.11) is that $C_{m}^{12}(z)=B_{m}^{12}(z) \neq O$ in this case. Therefore, in this case, we have to add $S_{0}^{12}(z) \neq 0$ which is uniquely solved in view of Lemma 4.5. The others are the same with the previous argument.

Remark 4.6. 1) Each of the matrix equations (4.11) and (4.15) is rewritten in the form of full rank system of the irregularity $p-q>0$ as we shall see below. By which we know that the classical asymptotic theory of divergent power series solution (cf. [Was]) and the theory of the $k$-summability or the multi-summability (cf. [Bra]) are applicable for the transformation matrices $R(z)$ and $S(z)$ (see other related papers [Bal1,2], [Lod1,2], [R-S], etc).

We consider the matrix equation $(4.11)$ for $R^{21}(z)$. The treatment for the equation of $S^{12}(z)$ is the same.

Let $T=\left\{t_{i}=s_{i} / N_{1}\right\} \subset \boldsymbol{Z} / N_{1}$ be the V-numbers associated with $V(C)=$ $q=m / N_{1} \in Z / N_{1}$. By putting $T^{(1)}=\left(t_{1}, \ldots, t_{N_{1}}\right)$ and $T^{(2)}=\left(t_{N_{1}+1}, \ldots, t_{N}\right)$, we define

$$
z^{T^{(1)}}=\operatorname{Diag}\left[z^{t_{1}}, \ldots, z^{t_{N_{1}}}\right], \quad z^{T^{(2)}}=\operatorname{Diag}\left[z^{t_{N_{1}+1}}, \ldots, z^{t_{N}}\right] .
$$

We transform the matrix functions $B^{i j}(z)$ and $R^{i j}(z)$ by

$$
z^{-T^{(j)}} B^{i j}(z) z^{T^{(j)}}=z^{m / N_{1}} \tilde{B}^{i j}\left(z^{1 / N_{1}}\right), \quad z^{-T^{(j)}} R_{\ell}^{i j}(z) z^{T^{(j)}}=\tilde{R}^{i j}\left(z^{1 / N_{1}}\right),
$$

with $\tilde{B}^{i j}(x), \tilde{R}^{i j}(x) \in M_{N}(\boldsymbol{C}[[x]])$, where

$$
\operatorname{det}\left(\tilde{B}^{11}(0)\right) \neq 0, \quad \tilde{B}^{21}(0)=O, \quad \tilde{B}^{22}(0)=\operatorname{Triang}[0, \ldots, 0] .
$$

Then a change of variable by $z^{1 / N_{1}}=x$ reduces the equation (4.11) into the form

$$
\begin{aligned}
\frac{1}{N_{1}} x^{\left(p N_{1}-m\right)+1} \frac{d}{d x} \tilde{R}^{21}(x)= & \left\{\tilde{\boldsymbol{B}}^{22}(x)+T^{(2)} x^{p N_{1}-m}\right\} \tilde{R}^{21}(x) \\
& -\tilde{R}^{21}(x)\left\{\tilde{\boldsymbol{B}}^{11}(x)+x^{p N_{1}-m} T^{(1)}\right\} \\
& +\tilde{B}^{21}(x)-\tilde{R}^{21}(x) \tilde{B}^{12}(x) \tilde{R}^{21}(x) .
\end{aligned}
$$

We introduce a vector function $\vec{X}(x)={ }^{\mathrm{t}}\left(X_{1}(x), \ldots, X_{M_{2} \times N_{1}}(x)\right) \in$ $\boldsymbol{C}[[x]]^{M_{2} \times N_{1}}$ which is defined by arranging the column vectors of $\tilde{\boldsymbol{R}}^{21}(x)$. Then we get the following system for $X(x)$.

$$
\begin{aligned}
\frac{1}{N_{1}} x^{\left(p N_{1}-m\right)+1} \frac{d}{d x} \vec{X}(x)= & \left\{\mathscr{A}_{0}+\mathscr{A}_{1}(x)\right\} \vec{X}(x)+\mathscr{A}_{2}(x) \\
& +\vec{F}\left(x, X_{i}, X_{j} ; 1 \leq i, j \leq M_{2} \times N_{1}\right),
\end{aligned}
$$


where $\mathscr{A}_{0}$ is a constant matrix with $M_{2}$-multiple eigenvalues each of which is a nonzero eigenvalues of $\tilde{B}^{11}(0), \vec{F}\left(x, X_{i}, X_{j} ; \ldots\right)$ is a vector function of quadratic nonlinearity and

$$
\operatorname{det}\left(\mathscr{A}_{0}\right) \neq 0, \quad \mathscr{A}_{1}(0)=O, \quad \mathscr{A}_{2}(0)=O .
$$

This shows that (4.17) is a standard system of full rank of the irregularity $p N_{1}-m>0$ in $x$ variable for which the classical theory of the asymptotic expansion in [Was] and also the results of the $k$-summability or the multisummability in $[\mathrm{Bra}]$ is applicable.

2) The transformation matrix

$$
R(z)=\left[\begin{array}{cc}
I_{N_{1}} & O \\
R^{21}(z) & I_{M_{2}}
\end{array}\right]
$$

is obtained by solving the equations (4.11) for $R^{21}(z)$, which is actually solved by (4.13) based on Lemma 4.5 .

We give another way to get $R(z)$, which is more primitive but direct than before.

First we define a matrix $R^{(1)}(z) \in G L_{N}(\mathscr{R}[z])$ with properties that $V\left(R^{(1)}\right)=0$ and $R_{0}^{(1)}(z)=I_{N}$ by

$$
R^{(1)}(z)=\left[\begin{array}{cc}
I_{N_{1}} & O \\
B_{m+1}^{21}(z)\left(B_{m}^{11}\right)^{-1}(z) & I_{M_{2}}
\end{array}\right] .
$$

Then for the reduced system by $R^{(1)}(z)$,

$$
R^{(1)}(z):(p, B(z)) \mapsto\left(p, B^{(2)}(z)\right), \quad B^{(2)}(z)=\left[\begin{array}{ll}
B^{(2)^{11}}(z) & B^{(2)^{12}}(z) \\
B^{(2)^{21}}(z) & B^{(2)^{22}}(z)
\end{array}\right],
$$

we see that $V\left(B^{(2)}\right)=V(B)$ and $B^{(2)}(z)$ has the same principal matrix $B_{m}(z)$. The difference is that the last row vector of $B_{m+1}^{(2)}{ }_{m+1}^{21}(z)$ vanishes. This is assured from the assumption that $B_{m}^{22}(z)=\operatorname{Triang}[0, \ldots, 0]$ is a strictly upper triangular matrix. Next we define a transformation matrix for $\left(p, B^{(2)}(z)\right)$ by

$$
R^{(2)}(z)=\left[\begin{array}{cc}
I_{N_{1}} & O \\
B_{m+1}^{(2)}(z)\left(B_{m}^{11}\right)^{-1}(z) & I_{M_{2}}
\end{array}\right],
$$

and continue the reduction procedure.

By this manner, we define a series of matrices $\left\{R^{(j)}\right\}_{j=1}^{\infty}$. Then we get the desired transformation matrix $R(z)$ by

$$
R(z)=R^{(1)}(z) R^{(2)}(z) R^{(3)}(z) \ldots
$$

Such a construction seems to be non-efficient than (4.13), but in actual numerical experiments this is more easy to calculate. (cf. Section 7 for some numerical experiments.) 


\subsection{Proof of Theorem $B$}

We prove Theorem $\mathrm{B}$ for the transformation matrix $P_{\delta}(z)$ in Theorem $\mathrm{A}_{\delta}$. The proof for $P_{\tau}(z)$ is obtained from the expression (4.25).

Before we proceed to the proof, we trace the reduction procedure to get $P_{\delta}(z)$ (cf. (4.23)).

We start from a singular system $L=(p, A(z))$. By applying Propositions 4.2, 4.3 and 4.4, we find matrices $Q_{1}(z) \in G L_{N}(\boldsymbol{C}[z])$,

$$
R_{1}(z)=\left[\begin{array}{cc}
I_{N_{1}} & O \\
R^{21}(z) & I_{M_{2}}
\end{array}\right] \in G L_{N}(\mathscr{R}[[z]]), \quad S_{1}(z)=\left[\begin{array}{cc}
I_{N_{1}} & S^{12}(z) \\
O & I_{M_{2}}
\end{array}\right] \in G L_{N}(\mathscr{R}[[z]])
$$

such that

$$
\begin{aligned}
P_{1}(z) & :=Q_{1}(z) R_{1}(z) S_{1}(z): L=(p, A(z)) \mapsto \\
L_{Q_{1} R_{1} S_{1}} & =\left(p, A_{1}(z)\right) \oplus\left(p, C^{22}(z)\right),
\end{aligned}
$$

with $V\left(A_{1}\right)=p-\rho(L)<V\left(C^{22}\right)$. Therefore, $L_{1}=\left(p, A_{1}(z)\right)$ is a full rank system of irregular singular type in Theorem $\mathrm{A}_{\delta}$.

Next we apply the same operations for the subsystem $\left(p, C^{22}(z)\right)$, and we get matrices $\tilde{Q}_{2}(z) \in G L_{M_{2}}(\boldsymbol{C}[z])$,

$$
\tilde{R}_{2}(z)=\left[\begin{array}{cc}
I_{N_{2}} & O \\
\tilde{R}^{21}(z) & I_{M_{3}}
\end{array}\right] \in G L_{M_{2}}(\mathscr{R}[[z]]), \quad \tilde{S}_{2}(z)=\left[\begin{array}{cc}
I_{N_{2}} & \tilde{S}^{12}(z) \\
O & I_{M_{3}}
\end{array}\right] \in G L_{M_{2}}(\mathscr{R}[[z]])
$$

such that

$$
\tilde{P}_{2}(z):=\tilde{Q}_{2}(z) \tilde{R}_{2}(z) \tilde{S}_{2}(z):\left(p, C^{22}(z)\right) \mapsto\left(p, A_{2}(z)\right) \oplus\left(p, C^{33}(z)\right),
$$

where $L_{2}=\left(p, A_{2}(x)\right)$ is a full rank system of irregular singular type in Theorem $\mathrm{A}_{\delta}$ and

$$
V\left(A_{1}\right)<V\left(C^{22}\right) \leq V\left(A_{2}\right)<V\left(C^{33}\right) .
$$

This means that by putting $Q_{2}(z)=I_{N_{1}} \oplus \tilde{Q}_{2}(z), R_{2}(z)=I_{N_{1}} \oplus \tilde{R}_{2}(z)$ and $S_{2}(z)=$ $I_{N_{1}} \oplus \tilde{S}_{2}(z)$ we have

$$
Q_{2} R_{2} S_{2}: L_{Q_{1} R_{1} S_{1}} \mapsto L_{\left(Q_{1} R_{1} S_{2}\right)\left(Q_{2} R_{2} S_{2}\right)}=L_{1} \oplus L_{2} \oplus\left(p, C^{33}(z)\right)
$$

By continuing these operations for the remainder subsystem $\left(p, C^{j j}(z)\right)(j \geq 3)$ and so on, we finally obtain the desired blocked diagonalization by irreducible subsystems, which is given by the following flow chart.

$$
(p, A(z)) \stackrel{P_{1}}{\rightarrow}\left(p, E_{1}(z)\right) \stackrel{P_{2}}{\rightarrow}\left(p, E_{2}(z)\right) \stackrel{P_{3}}{\rightarrow} \cdots \stackrel{P_{k}}{\rightarrow}\left(p, E_{k}(z)\right) \stackrel{Q_{k+1}}{\longrightarrow} \bigoplus_{i=1}^{k+1}\left(p, A_{i}(z)\right),
$$


where $P_{i}(z)=Q_{i}(z) R_{i}(z) S_{i}(z)$ and

$$
E_{i}(z)=\bigoplus_{j=1}^{i} A_{j}(z) \oplus C^{i+1, i+1}(z) .
$$

Now, the transformation matrix $P_{\delta}(z)$ in Theorem $\mathrm{A}_{\delta}$ is obtained by

$$
P_{\delta}(z)=P_{1}(z) P_{2}(z) \ldots P_{k}(z) Q_{k+1}(z) .
$$

Note that this is the case when $N_{k+1}>0$. If $N_{k+1}=0, P_{k}$ is replaced by $Q_{k}(z)$.

From the above observation we know that the transformation matrix $P_{\tau}(z) \in G L_{N}(\mathscr{R}[[z]])$ in Theorem $\mathrm{A}_{\tau}$ is obtained by

$$
P_{\tau}(z)=\left\{Q_{1}(z) R_{1}(z)\right\} \ldots\left\{Q_{k}(z) R_{k}(z)\right\} Q_{k+1}(z) .
$$

Proof of Theorem B. Theorem B for $P_{\delta}(z)$ is proved by estimating $O\left(P_{i}\right)$, $O\left(A_{i}\right)$ and $O\left(C^{i+1, i+1}\right)$ based upon (3.12) in Proposition 3.8 which relies on the span condition (3.6) in Lemma 3.4 (cf. (4.30)). Note that we get the same estimate with $O\left(P_{\delta}\right)$ for $O\left(P_{\tau}\right)$, since $R_{i}(z)$ and $R_{i}(z) S_{i}(z)$ have a common $\mathrm{V}$-numbers.

Recall that $M_{j}=N-\left(N_{1}+\cdots+N_{j-1}\right)(2 \leq j \leq k)$ and $M_{1}=N$. From the above observation we know that $C^{i i}(z) \in M_{M_{i}}(\mathscr{R}[[z]]) \quad\left(C^{11}(z)=A(z)\right)$.

Remember that the matrix $\tilde{Q}_{i}(z) \in M_{M_{i}}(C\{z\})$ sends the system $\left(p, C^{i i}(z)\right)$ into a non degenerate system, say, $\tilde{Q}_{i}(z):\left(p, C^{i i}(z)\right) \mapsto\left(p, B^{i i}(z)\right)$. Note that it holds that $O\left(C^{i i}\right) \leq O\left(B^{i i}\right)$ and $V\left(B^{i i}\right) \geq V\left(C^{i i}\right)$. By Lemma 3.4 (span condition) for the $\mathrm{V}$-numbers $T^{(i)}$ of $B^{i i}(z)$ we have

$$
\sigma\left(T^{(i)}\right) \leq\left(M_{i}-1\right)\left\{V\left(B^{i i}\right)-O\left(B^{i i}\right)\right\}<\left(M_{i}-1\right)\left\{p-O\left(C^{i i}\right)\right\},
$$

since $V\left(B^{i i}\right)<p(1 \leq i \leq k)$ and $O\left(C^{i i}\right) \leq O\left(B^{i i}\right)$. Note that $O\left(C^{11}\right)=O(A)$.

By applying Proposition 3.8 we get the following estimates for the order of zeros of $\tilde{R}_{i}(z)$ and $\tilde{R}_{i}(z) \tilde{S}_{i}(z)$,

$$
\min \left\{O\left(\tilde{R}_{i}\right), O\left(\tilde{R}_{i} \tilde{S}_{i}\right)\right\} \geq-\sigma\left(T^{(i)}\right) \geq-\left(M_{i}-1\right)\left\{p-O\left(C^{i i}\right)\right\} .
$$

Since $\tilde{Q}_{i} \tilde{R}_{i} \tilde{S}_{i}:\left(p, C^{i, i}(z)\right) \mapsto\left(p, A_{i}(z) \oplus C^{i+1, i+1}(z)\right)$ and that $\tilde{Q}_{i} \in G L_{M_{i}}(\boldsymbol{C}[z])$, we get the following order estimate by (3.12).

$$
\begin{aligned}
O\left(A_{i}(z) \oplus C^{i+1, i+1}(z)\right) & \geq-\sigma\left(T^{(i)}\right)+V\left(B^{i i}\right) \\
& \geq-\left(M_{i}-1\right)\left\{V\left(B^{i i}\right)-O\left(B^{i i}\right)\right\}+V\left(B^{i i}\right) \\
& >-\left(M_{i}-1\right)\left\{p-O\left(C^{i i}\right)\right\}+p,
\end{aligned}
$$

which shows that $\min \left\{O\left(A_{i}\right), O\left(C^{i+1, i+1}\right)\right\}>-\left(M_{i}-1\right)\left\{p-O\left(C^{i i}\right)\right\}+p$. 
We are now in the position to prove Theorem B inductively. First, we have

$$
\left\{\begin{array}{c}
\min \left\{O\left(P_{1}\right), O\left(R_{1} S_{1}\right)\right\}>-(N-1)\left\{p-O\left(C^{11}\right)\right\} \\
=-\left(M_{1}-1\right)\{p-O(A)\} \\
O\left(A_{1}\right) \geq-\left(M_{1}-1\right)\left\{V\left(C^{11}-O\left(C^{11}\right)\right\}+V\left(C^{11}\right)\right. \\
>-\left(M_{1}-1\right)\{p-O(A)\}+p \\
p-O\left(C^{22}\right)<\left(M_{1}-1\right)\{p-O(A)\}
\end{array}\right.
$$

Next, we examine $O\left(P_{2}\right), O\left(A_{2}\right)$ and $O\left(C^{33}\right)$; First,

$$
\begin{aligned}
\min \left\{O\left(P_{2}\right), O\left(R_{2} S_{2}\right)\right\} & >-\left(M_{2}-1\right)\left\{p-O\left(C^{22}\right)\right\} \\
& >-\left(M_{2}-1\right)\left(M_{1}-1\right)\{p-O(A)\} .
\end{aligned}
$$

Second,

$$
\begin{aligned}
O\left(A_{2}\right) & >-\left(M_{2}-1\right)\left\{p-O\left(C^{22}\right)\right\}+p \\
& >-\left(M_{2}-1\right)\left(M_{1}-1\right)\{p-O(A)\}+p .
\end{aligned}
$$

Third,

$$
p-O\left(C^{33}\right)<\left(M_{2}-1\right)\left\{p-O\left(C^{22}\right)\right\}<\left(M_{2}-1\right)\left(M_{1}-1\right)\{p-O(A)\} .
$$

Now by the induction principle, we get the desired estimates for $1 \leq i \leq$ $k-1$;

$$
\left\{\begin{array}{l}
O\left(P_{i}\right)>-\left(M_{i}-1\right) \ldots\left(M_{1}-1\right)\{p-O(A)\} \\
O\left(A_{i}\right)>-\left(M_{i}-1\right) \ldots\left(M_{1}-1\right)\{p-O(A)\}+p \\
p-O\left(C^{i+1, i+1}\right)<\left(M_{i}-1\right) \ldots\left(M_{1}-1\right)\{p-O(A)\} .
\end{array}\right.
$$

When $N_{k+1}=0$, the matrix function $A_{k}(z)$ is obtained by

$$
\tilde{Q}_{k}(z):\left(p, C^{k k}(z)\right) \mapsto\left(p, A_{k}(z)\right),
$$

which shows that $O\left(A_{k}\right) \geq O\left(A_{k-1}\right)$ since $O\left(A_{k-1}\right)$ and $O\left(C^{k k}\right)$ have the same estimate and $\tilde{Q}_{k}(z) \in M_{N_{k}}(\boldsymbol{C}[z])$.

When $N_{k+1}>0, A_{k}(z)$ and $A_{k+1}(z)$ are obtained by

$$
\begin{aligned}
& \tilde{P}_{k}(z):\left(p, C^{k k}(z)\right) \mapsto\left(p, A_{k}(z) \oplus C^{k+1, k+1}(z)\right), \\
& \tilde{Q}_{k+1}(z):\left(p, C^{k+1, k+1}(z)\right) \mapsto\left(p, A_{k+1}(z)\right),
\end{aligned}
$$

which prove the desired estimates for matrix functions. Finally $O\left(P_{\delta}\right)$ is estimated by

$$
O\left(P_{\delta}\right) \geq \sum_{i=1}^{k} O\left(P_{i}\right)>-\sum_{i=1}^{k}\left(M_{1}-1\right) \ldots\left(M_{i}-1\right)\{p-O(A)\} .
$$


Example 4.7 (basic idea of the reduction). For an easy understanding of the above idea, let us consider the following system,

$$
L=(3, A(z))=z^{4} D-A(z), \quad A(z)=\left[\begin{array}{ccc}
z & z^{3} & 1 \\
1 & 0 & 0 \\
z^{4} & 0 & 0
\end{array}\right] .
$$

It is easily examined that $V(A)=1$ and the associated V-numbers $T=\left\{t_{i}\right\}_{i=1}^{3}$ is determined from the inequalities (cf. (3.3), (3.4)),

$$
-2 \leq t_{2}-t_{1} \leq-1, \quad 1 \leq t_{3}-t_{1} \leq 3 .
$$

We take $T=\left\{t_{1}=2, t_{2}=0, t_{3}=4\right\}$, since $V(A)=1 \in Z$. Then $T$-expansion $A(z)=\sum_{\ell=0}^{1} A_{1+\ell}(z)$ is given by

$$
A_{1}(z)=\stackrel{\circ}{A}_{T}(z)=\left[\begin{array}{ccc}
z & z^{3} & 0 \\
0 & 0 & 0 \\
0 & 0 & 0
\end{array}\right], \quad A_{2}(z)=\left[\begin{array}{ccc}
0 & 0 & 1 \\
1 & 0 & 0 \\
z^{4} & 0 & 0
\end{array}\right] .
$$

Therefore, $L$ is of non degenerate of irregular singular type, but is not of full rank. Note that the $(1,2)$-entry $z^{3}$ in $A_{1}(z)$ does not play any role as a principal part. One can remove this term from the principal matrix function by taking the $\mathrm{V}$-numbers by $T^{\prime}=\left\{t_{1}^{\prime}=2, t_{2}^{\prime}=1 / 2, t_{3}^{\prime}=4\right\} \not \subset \boldsymbol{Z}$. But we take $T$ as before since $V(A)=1 \in Z$. Anyway we know that $N_{1}=1$ and $M_{2}=2$ in our case. To eliminate the $(2,1)$-block matrix ${ }^{\mathrm{t}}\left(1, z^{4}\right)$ from $A(z)$ or $A_{2}(z)$, we take a transformation matrix $R^{(1)}(z)$ defined by (4.18) in Remark 4.6-2),

$$
R^{(1)}(z)=\left[\begin{array}{cc}
I_{1} & O \\
A_{2}^{21}(z)\left(A_{1}^{11}\right)^{-1}(z) & I_{2}
\end{array}\right]=\left[\begin{array}{ccc}
1 & 0 & 0 \\
z^{-1} & 1 & 0 \\
z^{3} & 0 & 1
\end{array}\right] .
$$

Then the reduced system by this matrix becomes

$$
L_{R^{(1)}}=z^{4} D-\left[\begin{array}{ccc}
z+z^{2}+z^{3} & z^{3} & 1 \\
-z & -z^{2} & -z^{-1} \\
-z^{5}-4 z^{6} & -z^{6} & -z^{3}
\end{array}\right] .
$$

Next, in order to kill the term ${ }^{t}\left(-z,-z^{5}\right)$ on the $(2,1)$-block we take a transformation matrix by

$$
R^{(2)}(z)=\left[\begin{array}{ccc}
1 & 0 & 0 \\
-1 & 1 & 0 \\
-z^{4} & 0 & 1
\end{array}\right]
$$


By this matrix we get

$$
L_{R^{(1)} R^{(2)}}=z^{4} D-\left[\begin{array}{ccc}
z+z^{2}-z^{4} & z^{3} & 1 \\
2 z^{2}+z^{3}-z^{4} & -z^{2}+z^{3} & -z^{-1}+1 \\
-2 z^{6}+5 z^{7}-z^{8} & -z^{6}+z^{7} & -z^{3}+z^{4}
\end{array}\right]
$$

By continuing these operations, we finally kill $(2,1)$-block which is given by

$$
z^{4} D-\left[\begin{array}{ccc}
z+z^{2}+O\left(z^{4}\right) & z^{3} & 1 \\
0 & -z^{2}+z^{3}+O\left(z^{4}\right) & -z^{-1}+1+O(z) \\
0 & -z^{6}+O\left(z^{7}\right) & -z^{3}+z^{4}+O\left(z^{5}\right)
\end{array}\right] .
$$

Next we look at the subsystem of $2 \times 2$ which is obtained by removing the first row and column,

$$
\tilde{L}=z^{4} D-\left[\begin{array}{cc}
-z^{2}+z^{3}+O\left(z^{4}\right) & -z^{-1}+1+O(z) \\
-z^{6}+O\left(z^{7}\right) & -z^{3}+z^{4}+O\left(z^{5}\right)
\end{array}\right]=\left(3, C^{22}(z)\right) .
$$

This is a non degenerate system of irregular singular type of $V\left(C^{22}\right)=2$ or $\rho(\tilde{L})=1$. In order to kill $-z^{6}$ in the $(2,1)$ entry, we take a transformation matrix by

$$
\tilde{\boldsymbol{R}}^{(1)}(z)=\left[\begin{array}{cc}
1 & 0 \\
z^{4} & 1
\end{array}\right]
$$

which reduces the system $\tilde{L}$ into

$$
\tilde{L}_{\tilde{R}^{(1)}}=z^{4} D-\left[\begin{array}{cc}
-z^{2}+O\left(z^{4}\right) & -z^{-1}+1+O(z) \\
O\left(z^{7}\right) & O\left(z^{5}\right)
\end{array}\right] .
$$

By continuing the similar operations we can kill the $(2,1)$-entry and obtain a system of the form,

$$
z^{4} D-\left[\begin{array}{cc}
-z^{2}+O\left(z^{4}\right) & -z^{-1}+1+O(z) \\
0 & O\left(z^{4}\right)
\end{array}\right] .
$$

By this manner, we get an irreducible decomposition of the system $L$ by

$$
L \sim\left\{z^{4} D-z-z^{2}+O\left(z^{4}\right)\right\} \oplus\left\{z^{4} D+z^{2}+O\left(z^{4}\right)\right\} \oplus\left\{z^{4} D-O\left(z^{4}\right)\right\} .
$$

This decomposition shows that the fundamental matrix solution $W(z)$ of $L u=0$ is obtained in the form,

$$
W(z)=P(z) \exp \left[\begin{array}{ccc}
-z^{-2} / 2-z^{-1} & 0 & 0 \\
0 & z^{-1} & 0 \\
0 & 0 & 0
\end{array}\right], \quad P(z) \in G L_{3}(C[[z]]) .
$$


For the Newton polygon $\mathrm{N}(L)$ we have a decomposition that $\mathrm{N}(L)=$ $\mathrm{N}\left(z^{4} D-z\right)+\mathrm{N}\left(z^{4} D+z^{2}\right)+\mathrm{N}\left(z^{4} D\right)$, which shows that $\mathrm{N}(L)$ have three vertexes $(3,9),(2,7)$ and $(1,6)$. By Theorem $\mathrm{C}^{(\infty)}$ we know that the index $\chi\left(L, \mathscr{G}^{s}\right)$ have two discontinuous points $s=3 / 2$ and $s=2$ as a function on $s \geq 1$.

\subsection{Hukuhara-Turrittin's canonical form}

In this subsection we make clear the structure of transformation matrix $P(z) \in G L_{N}\left(\mathscr{R}\left[\left[z^{1 / r}\right]\right] \quad(\exists r \in N)\right.$ which reduces a system $L=(p, A(z))$ into the Hukuhara-Turrittin canonical form, or equivalently we shall see an exact structure of the formal fundamental matrix solution $W(z)$ of $L u(z)=0$ as we have seen in the above example. The transformation matrix $P(z)$ is obtained by continuing reduction procedures after getting the matrix $P_{\delta}(z) \in$ $G L_{N}(\mathscr{R}[[z]])$ in Theorem $\mathrm{A}_{\delta}$. The task is like peeling the peel of onion. We shall show a flow chart of the procedure of peeling one piece of peel, which is done to each irreducible subsystem $L_{i}=\left(p, A_{i}(z)\right)(1 \leq i \leq k)$ in the irreducible decomposition in Theorem $\mathrm{A}_{\delta}$. The procedure will be continued until we meet the core of onion which is consisted of first kind systems in $\mathrm{V}$-sense like $L_{k+1}=\left(p, A_{k+1}(z)\right)$.

[1st step] Blocked decomposition by irreducible subsystems (Theorem $\mathrm{A}_{\delta}$ ):

Let a system $L=(p, A(z)) \quad\left(A(z) \in M_{N}(\mathscr{R}[[z]])\right.$ be given. Find $P_{\delta}(z) \in$ $G L_{N}(\mathscr{R}[[z]])$ such that

$$
P_{\delta}: L=(p, A(z)) \mapsto \bigoplus_{i=1}^{k+1} L_{i}, \quad L_{i}=\left(p, A_{i}(z)\right),
$$

becomes an irreducible decomposition with $V\left(A_{1}\right)<V\left(A_{2}\right)<\cdots<V\left(A_{k}\right)<$ $p \leq V\left(A_{k+1}\right)$.

[2nd step] For each irreducible factor:

(1) For the subsystem of first kind in V-sense

We take the V-numbers $T_{k+1}=\left\{t_{\ell}^{(k+1)}\right\}_{\ell=1}^{N_{k+1}} \subset \boldsymbol{Z}$ of $A_{k+1}(z)$ associated with $p\left(\leq V\left(A_{k+1}\right)\right)$. Then

$$
z^{T_{k+1}}: L_{k+1} \mapsto \tilde{L}_{k+1}=z^{p+1} D-z^{p} B_{k+1}(z), \quad B_{k+1}(z) \in M_{N_{k+1}}(C[[z]]),
$$

which is a singular system of first kind in usual sense. Recall that $z^{T}:=$ $\operatorname{Diag}\left[z^{t_{1}}, \ldots, z^{t_{N}}\right]$.

The reduction ends here for this factor.

(2) For the subsystem of full rank of irregular singular type

For $L_{i}=\left(p, A_{i}(z)\right)(1 \leq i \leq k)$, since we can take $V\left(A_{i}\right)=m_{i} / N_{i} \in \boldsymbol{Z} / N_{i}$, we take the associated V-numbers so that $T_{i}=\left\{t_{j}^{(i)}=s_{j}^{(i)} / N_{i}\right\}_{j=1}^{N_{i}} \subset \boldsymbol{Z} / N_{i}$. Then

$$
z^{T_{i}}: L_{i} \mapsto \tilde{L}_{i}=z^{p+1} D-z^{V\left(A_{i}\right)} C_{i}\left(z^{1 / N_{i}}\right), \quad C_{i}(t) \in M_{N_{i}}(\boldsymbol{C}[[t]]),
$$


with $C_{i}(0)=\left(\stackrel{\circ}{A}_{i}\right)_{T_{i}}$ for the principal matrix and $\operatorname{det} C_{i}(0) \neq 0$. The Poincaré rank is reduced to $p-V\left(A_{i}\right)$.

The reduction will be continued below for this factor.

[3rd step] Change of variable by fractional power:

A change of variable $t=z^{a}(a \in C)$ is denoted by $\left.\right|_{t=z^{a}}$. Then we have

$$
\left.\right|_{t=z^{1 / N_{i}}}: \tilde{L}_{i}\left(z, D_{z}\right) \mapsto \tilde{L}_{i}\left(t, D_{t}\right)=\frac{1}{N_{i}} t^{p N_{i}+1} D_{t}-t^{m_{i}} C_{i}(t), \quad p N_{i}>m_{i} .
$$

Here, it should be mentioned that the number $N_{i}$ may not be best possible. Exactly saying, $V\left(A_{i}\right)$ is taken in $\boldsymbol{Z} / r_{i}$ by some $r_{i}$ a divisor of $N_{i}$. If one wants to take a common integer $r$ for every $L_{i}(1 \leq i \leq k)$, we take $r$ the least common multiple of the denominators of $V\left(A_{i}\right)(1 \leq i \leq k)$.

[4th step] Constant transformation matrix and sub-sub-blocked diagonalization:

Let $\left\{\alpha_{\ell}^{(i)}\right\}_{\ell=1}^{n_{i}}$ be the distinct eigenvalues of $C_{i}(0)$, and $N_{\ell}^{(i)}$ be the multiplicity of $\alpha_{\ell}^{(i)}\left(N_{i}=\sum_{\ell=1}^{n_{i}} N_{\ell}^{(i)}\right)$. Suppose that $C_{i}(0)$ is transformed into a Jordan canonical form by a constant matrix $D_{i} \in G L_{N_{i}}(\boldsymbol{C})$ which we denote by $J_{i}=\operatorname{Diag}\left[J\left(\alpha_{1}^{(i)}\right), \ldots, J\left(\alpha_{n_{i}}^{(i)}\right)\right]$. Here $J\left(\alpha_{\ell}^{(i)}\right) \in M_{(i)} N_{\ell}^{(i)}(\boldsymbol{C})$ denotes the totality of Jordan blocks associated with the eigenvalue $\alpha_{\ell}^{(i)}$.

By the well known reduction procedure ([Was, Th. 11.1]), $D_{i}^{-1} \tilde{L}_{i}\left(t, D_{t}\right) D_{i}$ is reduced into a blocked diagonalized form corresponding to the Jordan decomposition $J_{i}$, that is, we can find $Q_{i}(t) \in G L_{N_{i}}(\boldsymbol{C}[[t]])$ such that

$$
D_{i} Q_{i}(t): \tilde{L}_{i}\left(t, D_{t}\right) \mapsto \mathscr{L}_{i}=\bigoplus_{\ell=1}^{n_{i}} \mathscr{L}_{\ell}^{(i)}\left(t, D_{t}\right),
$$

with

$$
\begin{aligned}
& \mathscr{L}_{\ell}^{(i)}\left(t, D_{t}\right)=\frac{1}{N_{i}} t^{p N_{i}+1} D_{t}-t^{m_{i}} B_{\ell}^{(i)}(t), \\
& B_{\ell}^{(i)}(t) \in M_{N_{\ell}^{(i)}}(C[[t]]), \quad B_{\ell}^{(i)}(0)=J\left(\alpha_{\ell}^{(i)}\right) .
\end{aligned}
$$

[5th step] Exponential transformation:

We define

$$
\begin{aligned}
& \exp \left[\Lambda_{i}(t)\right]=\exp \left[\operatorname{Diag}\left[\Lambda_{1}^{(i)}(t), \ldots, \Lambda_{n_{i}}^{(i)}(t)\right]\right] \\
& \Lambda_{\ell}^{(i)}(t)=\frac{N_{i} \alpha_{\ell}^{(i)}}{m_{i}-p N_{i}} t^{-\left(p N_{i}-m_{i}\right)} \cdot I_{N_{\ell}^{(i)}} .
\end{aligned}
$$

Then

$$
\exp \left[\Lambda_{i}(t)\right]: \bigoplus_{\ell=1}^{n_{i}} \mathscr{L}_{\ell}^{(i)}\left(t, D_{t}\right) \mapsto \bigoplus_{\ell=1}^{n_{i}} \tilde{\mathscr{L}}_{\ell}^{(i)}\left(t, D_{t}\right)
$$


where

$$
\tilde{\mathscr{L}}_{\ell}^{(i)}\left(t, D_{t}\right)=\frac{1}{N_{i}} t^{p N_{i}+1} D_{t}-t^{m_{i}} \mathscr{A}_{i}(t), \quad \mathscr{A}_{\ell}^{(i)}(t)=B_{\ell}^{(i)}(t)-\alpha_{\ell}^{(i)} I_{N_{\ell}^{(i)}}
$$

The leading term $\mathscr{A}_{\ell}^{(i)}(0)=J\left(\alpha_{\ell}^{(i)}\right)-\alpha_{\ell}^{(i)} I_{N_{\ell}^{(i)}}$ is a Jordan canonical form of a nilpotent matrix, that is, a triangular matrix in strict sense. This shows that

$$
O\left(t^{m_{i}} B_{\ell}^{(i)}(t)\right)=V\left(t^{m_{i}} B_{\ell}^{(i)}\right)=t^{m_{i}}<V\left(t^{m_{i}} \mathscr{A}_{\ell}^{(i)}\right),
$$

which turns back to the situation of the 1-st step.

Summary 4.8. For a singular system $L=(p, A(z))$, at each stair in the reduction procedures into Hukuhara-Turrittin's canonical form, the transformation matrix is taken in the following form.

$$
\left.P_{\delta}(z) z^{\left\{T_{i}\right\}}\right|_{t=z^{\left\{1 / N_{i}\right\}}} D Q(t) \exp [\Lambda(t)],
$$

with



We finally meet a core of the system which is consisted of the first kind systems in $V$-sense.

\section{Index formula of system on formal Gevrey space}

We shall prove an index formula of the mapping (2.8), $L=(p, A(z))$ : $\left(\mathscr{G}^{s}\right)^{N} \rightarrow\left(\mathscr{G}^{S}\right)^{N}(s \geq 1)$ in case $A(z) \in M_{N}(\boldsymbol{C}\{z\})$. Precisely, the index is obtained from the vertical coordinate of the associated vertex of the Newton polygon $\mathrm{N}(L)$ as $(1.9)$ for a single operator $P(z, D)$.

First, we note that the index formula in case $s=1$ that $\chi\left(L ; \mathscr{G}^{1}\right)=-N p$ $\left(\mathscr{G}^{1} \equiv \boldsymbol{C}\{z\}\right)$ was already proved by H. Komatsu [Kom] (cf. [Miy2] for more general system). Note that $(N, N p)$ is the highest vertex of $\mathrm{N}(L)$ in $y$-axis. In case $s=\infty$, the formula that $\chi\left(L ; \mathscr{G}^{\infty}\right)=-\sum_{j=1}^{k+1} N_{j} V\left(A_{j}\right)$ with $V\left(A_{k+1}\right)=p$ (cf. Theorem $\mathrm{A}_{\delta}$ ) is easily obtained from [Miy2] combined with the argument below in case $1<s<\infty$. By these reasons our proof will be restricted in case $1<s<\infty$. 
We have to mention that the proof will be done by applying the index formula proved in a joint paper with M. Yoshino [M-Y2] where the determinant theory over the ring of differential operators was employed. The idea of applying the theory in [M-Y2] to our system was given in a paper [M-I, Th. B and Sec. 7], in which it was shown that the first discontinuous point of $\chi\left(L: \mathscr{G}^{S}\right)$ in $s \geq 1$ is given by $s=1+1 / \rho(L)$. This result is nothing but a characterization of the steepest slope $\rho(L)$ of $\mathrm{N}(L)$ in the word in this paper.

\subsection{Short review of results in [M-Y2]}

We define a function space $\mathscr{G}^{s}(w)$ by

$$
\mathscr{G}^{s}(w) \ni u(z)=\sum_{n=0}^{\infty} u_{n} \frac{z^{n}}{n !} \quad \stackrel{\text { def. }}{\Longleftrightarrow}\left(\hat{\mathscr{B}}_{s}\right)(\zeta):=\sum_{n=0}^{\infty} u_{n} \frac{\zeta^{n}}{(n !)^{s}} \in \mathcal{O}(w),
$$

where $\mathcal{O}(w)$ denotes the set of holomorphic functions on an open disk $B_{w}=$ $\{\zeta \in C ;|\zeta|<w\}$ equipped with a Fréchet space topology of uniform convergence on every compact set in $B_{w}$. Therefore $\mathscr{G}^{s}(w)$ has a Fréchet space topology induced from $\mathcal{O}(w)$. It is obvious that $\mathscr{G}^{s}=\bigcup_{w>0} \mathscr{G}^{s}(w)$. In order to investigate properties of differential operator $z^{j} D^{k}$ on $G^{s}(w)$, we introduce a Banach space $G_{\mu}^{s}(w)$ by

$$
G_{\mu}^{s}(w) \ni u(z)=\sum_{n=0}^{\infty} u_{n} \frac{z^{n}}{n !} \quad \stackrel{\text { def. }}{\Longleftrightarrow}\|u\|_{w ; \mu}^{(s)}:=\sum_{n=0}^{\infty}\left|u_{n}\right| \frac{\left(s^{s} w\right)^{n}}{(s n+\mu) !}<\infty .
$$

It is obvious that $G^{s}(w)$ is equipped with the projective limit of $G_{\mu}^{s}(r)$ as $r \nearrow w$ for any fixed $\mu \geq 0, \mathscr{G}^{s}(w)=\operatorname{proj} \lim _{r / w} G_{\mu}^{(s)}(r)$.

In [M-Y2], we investigated an index formula for the following mapping which is more precise than (2.8),

$$
L:\left(\mathscr{G}^{s}(w)\right)^{N} \rightarrow\left(\mathscr{G}^{s}(w)\right)^{N}, \quad w>0 .
$$

But the study was restricted in the case of polynomial coefficients $A(z) \in$ $M_{N}(\boldsymbol{C}[z])$. Actually, in [M-Y2], general square matrix of differential operators with polynomial coefficients was studied, and the index formula was obtained by the winding number of an associated symbol around the origin, which is formulated in the below.

Definition 5.1 (Gevrey order, Gevrey principal symbol). Let $1<s<\infty$ be fixed.

1) For an operator $z^{j} D^{k}$, we define the $s$-Gevrey order by $\mu_{s}\left(z^{j} D^{k}\right):=$ $(1-s) j+s k$. For a zero operator, we define $\mu_{s}(0):=-\infty$. 
2) For an operator $p=p(z, D) \in W(\boldsymbol{C})$, the differential operators of polynomial coefficients, which we denote by

$$
p=\sum_{j, k \geq 0}^{\text {finite }} a_{j k} z^{j} D^{k} \quad\left(a_{j k} \in \boldsymbol{C}\right),
$$

the $s$-Gevrey order $\mu_{s}(p) \in \boldsymbol{R}$ is defined by

$$
\mu_{s}(p)=\max \left\{(1-s) j+s k ; a_{j k} \neq 0\right\},
$$

and the $s$-Gevrey principal symbol $p_{s}(z, \zeta)$ is defined by

$$
p_{s}(z, \zeta)=\sum_{\mu_{s}\left(x^{j} D^{k}\right)=\mu_{s}(p)} a_{j k} z^{j} \zeta^{k} .
$$

3) For a matrix of operators $P=\left(p_{i j}(z, D)\right) \in M_{N}(W(C))$, the total $s$-Gevrey order $\mu_{s}(P) \in \boldsymbol{R} \cup\{-\infty\}$ is defined by

$$
\mu_{s}(P)=\max _{\sigma \in S_{N}} \sum_{i=1}^{N} \mu_{s}\left(p_{i, \sigma(i)}\right) .
$$

Lemma 5.2 (Volevič). For $P=\left(p_{i j}(z, D)\right) \in M_{N}(W(\boldsymbol{C}))$, let assume $\mu_{s}(P) \in \boldsymbol{R}$ (resp. Q). Then there are systems of numbers $S=\left\{s_{i}\right\}_{i=1}^{N}, \quad T=$ $\left\{t_{j}\right\}_{j=1}^{N} \subset \boldsymbol{R}$ (resp. Q) such that

$$
\mu_{s}(P)=\sum_{i=1}^{N} s_{i}-\sum_{j=1}^{N} t_{j}, \quad \mu_{s}\left(p_{i j}\right) \leq s_{i}-t_{j}(\forall i, j=1,2, \ldots, N) .
$$

If $\sum_{i=1}^{N} \mu_{s}\left(p_{i i}\right)=\mu_{s}(P)$, then there is a system of numbers $T=\left\{t_{i}\right\}$ such that

$$
\mu_{s}\left(p_{i j}\right) \leq t_{i}-t_{j}+\mu_{s}\left(p_{i i}\right), \quad \forall i, j=1,2, \ldots, N .
$$

In this case $S=\left\{s_{i}=t_{i}+\mu_{s}\left(p_{i i}\right)\right\}$.

The proof of (5.8) is done by reducing it to Lemma 3.1, although the inequality is opposite direction. (cf. [M-I, Proposition 7.1]).

For a matrix $P=\left(p_{i j}(z, D)\right) \in M_{N}(W(\boldsymbol{C}))$, let $p_{i j}^{(s)}(z, \zeta)$ denote the $s$-Gevrey principal symbol of $p_{i j}(z, D)$, and put

$$
\stackrel{\circ}{p}_{i j}(z, \zeta)= \begin{cases}p_{i j}^{(s)}(z, \zeta), & \text { if } \mu_{s}\left(p_{i j}\right)=s_{i}-t_{j}, \\ 0, & \text { if } \mu_{s}\left(p_{i j}\right)<s_{i}-t_{j} .\end{cases}
$$

Definition 5.3 ( $s$-Gevrey principal matrix, $s$-Gevrey symbol, non degeneracy).

1) A matrix $\stackrel{\circ}{P}_{s}(z, \zeta)=\left(\stackrel{\circ}{p}_{i j}(z, \zeta)\right)$ is called the $s$-Gevrey principal matrix, and the $s$-Gevrey principal symbol $\stackrel{\circ}{p}_{s}(z, \zeta)$ is defined

$$
\stackrel{\circ}{p}_{s}(z, \zeta)=\operatorname{det} \stackrel{\circ}{P}_{s}(z, \zeta) \quad\left(=\sum_{(1-s) j+s k=\mu_{s}(P)} c_{j k} z^{j} \zeta^{k}\right) .
$$

2) When $\stackrel{\circ}{p}_{s}(z, \zeta) \not \equiv 0, P(z, D)$ is called of non degenerate type in $s$-Gevrey order. 
The definition of non degeneracy is redefined as follows:

For a polynomial $p(z, \zeta)=\sum_{j, k}^{\text {finite }} a_{j k} z^{j} \zeta^{k}$, we define the $s$-Gevrey order as in case of differential operator $p(z, D)$, which is denoted by $\mu_{s}(p)$ again.

For a matrix of operators $P=\left(p_{i j}(z, D)\right) \in M_{N}(W(\boldsymbol{C}))$, let $\mu_{s}(P) \in \boldsymbol{R}$. Then $P$ is of non degenerate type in $s$-Gevrey order if and only if

$$
\mu_{s}(\operatorname{det} P(z, \zeta))=\mu_{s}(P)
$$

Moreover, the $s$-Gevrey principal symbol is given by

$$
\begin{aligned}
\stackrel{\circ}{p}_{s}(z, \zeta)= & \text { collection of monomials in } \\
& \operatorname{det} P(z, \zeta) \text { of } s \text {-Gevrey order } \mu_{s}(P) .
\end{aligned}
$$

Now the following index formula was proved in [M-Y2] in more general situation.

Theorem 5.4 ([M-Y2, Th. I] (cf. [M-Y1]). Let $P \in M_{N}(W(C))$ be of non degenerate type in s-Gevrey order. Then the mapping (5.3) has an index

$$
-\chi\left(P ; \mathscr{G}^{s}(w)\right)=\lim _{r \nearrow w} \frac{1}{2 \pi i} \oint_{|z|=r} d \log \stackrel{\circ}{p}_{s}\left(z, z^{-1}\right) .
$$

Here the right hand side denotes the winding number of an oriented curve $\stackrel{\circ}{p}_{s}\left(z, z^{-1}\right)(|z|=r)$ around the origin for $r$ such that $\stackrel{\circ}{p}_{s}\left(z, z^{-1}\right) \neq 0$ on $\{|z|=r\}$.

The theorem shows that for a fixed $s>1$, the discontinuity of $\chi\left(L, \mathscr{G}^{s}(w)\right)$ on $w>0$ occurs at $w$ when $\stackrel{\circ}{p}_{s}\left(z, z^{-1}\right)$, which was called the Toeplitz symbol, have zeros on $|z|=w$.

\subsection{Proof of Theorems $\mathbf{C}^{(\infty)}$ and $\mathbf{C}^{(m)}$}

We assume that $A(z) \in M_{N}(\boldsymbol{C}[z])$ until 3rd step, which will be removed at 4 th step.

1st step (irreducible system):

For $A(z)=\left(a_{i j}(z)\right) \in M_{N}(\boldsymbol{C}[z])$, let $T=\left\{t_{i}\right\}$ be the V-numbers associated with $V(A)(\leq p)$, that is, $O\left(a_{i j}\right) \geq t_{i}-t_{j}+V(A)$. (In case $V(A) \geq p$, we understand that $V(A)=p$.) Hence for a fixed $s>1$,

$$
\mu_{s}\left(z^{p+1} D\right)=(1-s) p+1
$$

and

$$
\mu_{s}\left(a_{i j}\right) \begin{cases}=(1-s)\left(t_{i}-t_{j}+V(A)\right), & \text { if } \stackrel{\circ}{a}_{i j} \neq 0 \\ <(1-s)\left(t_{i}-t_{j}+V(A)\right), & \text { otherwise. }\end{cases}
$$


Imagine that $\mu_{s}\left(a_{i i}\right)=(1-s) V(A)$. Then

$$
(1-s) p+1=(1-s) V(A) \quad \Longleftrightarrow \quad(s-1)(p-V(A))=1 .
$$

1) (first kind system in V-sense):

The equality (5.13) is impossible when $V(A)=p$, that is, the case of first kind system in V-sense. In this case we always have $\mu_{s}\left(z^{p+1} D\right)>\mu_{s}\left(a_{i i}\right)$ for all $s>1$ and $i=1, \ldots, N$. This shows that

$$
\mu_{s}(L)=N(1-s) p+N, \quad \stackrel{\circ}{L}_{s}(z, \zeta)=z^{p+1} \zeta I_{N} .
$$

Therefore $L$ is a non degenerate system in $s$-Gevrey order with an $s$-Gevrey principal symbol,

$$
\stackrel{\circ}{\ell}_{s}(z, \zeta)=\operatorname{det} \stackrel{\circ}{L}_{s}(z, \zeta)=z^{N p}(z \zeta)^{N} .
$$

Then, by applying Theorem 5.4, we have

$$
\chi\left(L ; \mathscr{G}^{s}(w)\right)=-\frac{1}{2 \pi i} \oint_{|z|=w} d \log \stackrel{\circ}{\ell}_{s}\left(z, z^{-1}\right)=-p N \quad(\forall s>1, \forall w>0) .
$$

2) (full rank system of irregular singular type):

Let a system $L=(p, A(z))$ be a full rank system of irregular singular type, that is,

$$
0 \leq V(A)<p \quad \text { and } \quad \operatorname{det} \stackrel{\circ}{A}_{T}(z)=z^{N V(A)} \operatorname{det} \stackrel{\circ}{A}_{T} \neq 0 .
$$

In this case, (5.13) holds when

$$
s=1+\frac{1}{p-V(A)},
$$

where $p-V(A)=\rho(L)>0$ is the slope of only one non trivial side of $\mathrm{N}(L)$, and $s$ is the number given in (1.7). From the above argument, we see that $L$ is non degenerate in $s$-Gevrey order with the following $s$-Gevrey principal symbol $\ell_{s}(z, \zeta)$ according to the value $s>1$,

$$
\left\{\begin{array}{l}
\operatorname{det}\left(z^{p+1} \zeta I_{N}\right)=z^{N p}(z \zeta)^{N}, \quad 1<s<1+1 / \rho(L), \\
\operatorname{det}\left(z^{p+1} \zeta I_{N}-\stackrel{\circ}{T}_{T}(z)\right)=z^{p N}(z \zeta)^{N}+\cdots+(-1)^{N} \operatorname{det} \stackrel{\circ}{A}_{T}(z), \\
\quad s=1+1 / \rho(L), \\
\operatorname{det}\left(-\stackrel{\circ}{A}_{T}(z)\right)=(-1)^{N} z^{N V(A)} \operatorname{det} \stackrel{\circ}{A}_{T}, \quad s>1+1 / \rho(L),
\end{array}\right.
$$

where $\cdots$ on the middle line denotes a linear sum of the terms,

$$
z^{r_{j}} z^{p(N-j)}(z \zeta)^{N-j}, \quad(1-s)\left\{r_{j}+p(N-j)\right\}+N-j=(1-s) N V(A)
$$


for all $1 \leq j \leq N-1$ which imply $r_{j}=j V(A) \in N$. This shows, by Theorem 5.4 , that

$$
\chi\left(L, \mathscr{G}^{s}(w)\right)=\left\{\begin{array}{l}
-N p, \quad 1<s<1+1 /(p-V(A)), \forall w>0, \\
-\lim _{r / w} \frac{1}{2 \pi i} \oint_{|z|=r} d \log \stackrel{\circ}{\ell}_{s}\left(z, z^{-1}\right), \quad s=1+1 / \rho(L), \\
-N V(A), \quad 1+1 /(p-V(A)) \leq s<\infty, \forall w>0 .
\end{array}\right.
$$

Hence, when $s=1+1 / \rho(L)$ we have

$$
\left\{\begin{array}{l}
\lim _{w \searrow 0} \chi\left(L ; \mathscr{G}^{s}(w)\right)=-p N=\chi\left(L ; \mathscr{G}^{s}\right), \\
\lim _{w>\infty} \chi\left(L ; \mathscr{G}^{s}(w)\right)=-V(A) N .
\end{array}\right.
$$

2nd step (in case $P_{\tau}(z) \in G L_{N}(\boldsymbol{C}[[z]])$ ):

Let the transformation matrix $P_{\tau}(z)$ in Theorem $\mathrm{A}_{\tau}$ have no pole singularity,

$$
P_{\tau}(z): L \mapsto L_{P_{\tau}}=\left(p, \operatorname{Triang}\left[A_{1}(z), \ldots, A_{k}(z), A_{k+1}(z)\right]\right),
$$

where the subsystems $L_{i}=\left(p, A_{i}(z)\right) \quad(1 \leq i \leq k+1)$ are irreducible with a condition

$$
0 \leq V\left(A_{1}\right)<\cdots<V\left(A_{k}\right)<p \leq V\left(A_{k+1}\right) .
$$

We truncate the reduction procedure to get $P_{\tau}(z)$ in finite time, which becomes a polynomially invertible matrix, so that the reduced system satisfy the following conditions. For any fixed $m \geq N p$, the reduced system $L^{(m)}(z, D)$ has the following form,

$$
L^{(m)}=\left(p,\left(A_{i j}^{(m)}(z)\right)_{i, j=1, \ldots, k+1}\right), \quad O\left(A_{i j}^{(m)}\right) \geq m \quad \text { for } i>j,
$$

where the blocked matrix $A_{i j}^{(m)}(z) \in M_{N_{i} \times N_{j}}(\boldsymbol{C}[z])$ satisfies the following conditions;

1) $V\left(A_{k+1, k+1}^{(m)}\right) \geq p$

2) For each $A_{i i}^{(m)}(z)$ and $A_{i}(z)$ in $(5.19)(1 \leq i \leq k), V\left(A_{i i}^{(m)}\right)=V\left(A_{i}\right)$ and they have a common $\mathrm{V}$-numbers $T^{(i)}$ with the same principal matrix $\left(\AA_{i}\right)_{T^{(i)}}$.

The condition that $m \geq p N$ implies

$$
\begin{aligned}
\mu_{s}\left(z^{p N}\right)=(1-s) p N & <\max \{(1-s) p N+N,(1-s) N V(A)\} \\
& \leq \max \left\{\mu_{s}\left(z^{p+1} D I_{N}\right), \sum_{i=1}^{k+1} \mu_{s}\left(A_{i}\right)\right\} .
\end{aligned}
$$


This shows that the entries from $A_{i j}^{(m)}(z)(i>j)$ do not contribute to the $s$-Gevrey principal matrix or the symbol, since each blocked matrix $A_{i j}^{(m)}(z)$ $(i \leq j)$ has no pole singularity (cf. (5.11)). This implies that the reduced system $L^{(m)}(z, D)$ is non degenerate in $s$-Gevrey order and its $s$-Gevrey principal symbol $\hat{\ell}_{s}(z, \zeta)$ is given by that for $\bigoplus_{i=1}^{k+1} L_{i}(z, D)$,

$$
\stackrel{\circ}{\ell}_{s}(z, \zeta)=\prod_{i=1}^{k+1} \operatorname{det}\left(\stackrel{\circ}{L}_{i}\right)_{s}(z, \zeta) .
$$

This gives an index formula, in view of Theorem 5.4, that

$$
\chi\left(L ; \mathscr{G}^{s}(w)\right)=\chi\left(L^{(m)} ; \mathscr{G}^{s}(w)\right)=\sum_{i=1}^{k+1} \chi\left(L_{i} ; \mathscr{G}^{s}(w)\right), \quad(1<s<\infty, w>0) .
$$

which we want to prove.

3rd step (in case $P_{\tau}(z) \in G L_{N}(\mathscr{R}[[z]])$ :

We consider the case of $O\left(P_{\tau}\right)=-r<0$. Note that $r$ is estimated from above in Theorem B and Remark 2.1-2).

Now we recall the expression (4.25) which gives the exact structure of $P_{\tau}(z)$. By this expression we see that the pole singularity come from the triangular matrices $R_{j}(z)$. Note that we have $O\left(R_{j}\right)=O\left(R_{j}^{-1}\right)$ for the order of zeros or the pole order. By putting $-r_{j}=O\left(R_{j}\right) \leq 0$, we have $z^{r_{j}} R_{j}^{ \pm 1}(z) \in$ $M_{N}(\boldsymbol{C}[[z]])$. Therefore for $r=\sum_{j=1}^{k} r_{j}$, we have $z^{r} P_{\tau}^{ \pm 1}(z) \in M_{N}(\boldsymbol{C}[[z]])$ and

$$
\left(z^{r} P_{\tau}^{-1}(z)\right) L(z, D)\left(P_{\tau}(z) z^{r}\right)=z^{r} L_{P_{\tau}} z^{r}=(p+2 r, B(z)), \quad B(z) \in M_{N}(C[[z]]) .
$$

This is only a formal observation, and let us return to the actual proof.

We take and fix a number $m \in N$ such that $m+2 r \geq(p+2 r) N$, that is, $m \geq p N+(N-1) 2 r$. We take $P_{m}(z) \in G L_{N}(\mathscr{R}[z])$ like (5.20), which is obtained by truncating the procedure to get $P_{\tau}(z)$ in finite time, which means that

$$
\begin{aligned}
& P_{m}(z): L \mapsto L^{(m)}=\left(p,\left(A_{i j}^{(m)}(z)\right)\right), \\
& A_{i j}^{(m)}(z) \in M_{N_{i} \times N_{j}}(\mathscr{R}[z]), \quad O\left(A_{i j}^{(m)}\right) \geq m(i>j) .
\end{aligned}
$$

Then, from the above observation, we get that

$$
\begin{aligned}
\left(z^{r} P_{m}^{-1}(z)\right) L(z, D)\left(P_{m}(z) z^{r}\right) & =z^{r} L^{(m)} z^{r}=z^{p+2 r+1} D-\left(z^{2 r} A_{i j}^{(m)}(z)\right)+r z^{2 r+p} I_{N} \\
& =:\left(p+2 r,\left(B_{i j}^{(m)}(z)\right)\right), \quad\left(B_{i j}^{(m)}(z)\right) \in M_{N}(\boldsymbol{C}[z]),
\end{aligned}
$$

where $O\left(B_{i j}^{(m)}\right) \geq 2 r+m$ for $i>j$. From the condition for the number $m$, we know that the argument in the 2nd step does hold. Note that $z^{r_{j}} R_{j}(z)$ is a triangular matrix with diagonal entry $z^{r_{j}}$ which shows that this is of non 
degenerate in $s$-Gevrey order and its $s$-Gevrey principal symbol is $z^{N r_{j}}$. This shows that $\chi\left(z^{r_{j}} R_{j}(z) ; \mathscr{G}^{s}(w)\right)=-N r_{j}$. (Precisely speaking, we truncate the higher order part of the Taylor expansion from $R_{j}(z)$.) Keeping this in mind, by using the additivity of indexes, we have

$$
\begin{aligned}
\chi\left(\left(z^{r} P_{m}^{-1}\right) L\left(P_{m} z^{r}\right) ; \mathscr{G}^{s}(w)\right) & =\chi\left(z^{r} P_{m}^{-1} ; \mathscr{G}^{s}(w)\right)+\chi\left(L ; \mathscr{G}^{s}(w)\right)+\chi\left(P_{m} z^{r} ; \mathscr{G}^{s}(w)\right) \\
& =-2 r N+\chi\left(L ; \mathscr{G}^{s}(w)\right) .
\end{aligned}
$$

Here it should be remarked that $\mathrm{N}\left(z^{m} L\right)=\mathrm{N}\left(L z^{m}\right)=\mathrm{N}(L)+(0, m N)$, because the multiplication by a scalar function $z^{m}$ causes a vertical shift of $\mathrm{N}(L)$ by $m N$ which follows from the definition of the Newton polygon. Hence we have

$$
\mathrm{N}\left(\left(z^{r} P_{m}^{-1}\right) L\left(z^{r} P_{m}\right)\right)=\mathrm{N}\left(z^{r} I_{N}\right)+\mathrm{N}\left(L_{P_{m}}\right)+\mathrm{N}\left(z^{r} I_{N}\right)=\mathrm{N}(L)+2(0, r N),
$$

which implies the desired index formula.

4th step (removability of the assumption of polynomial coefficients):

We shall prove that Theorem 5.4 does hold even when the assumption $A(z) \in M_{N}(\boldsymbol{C}[z])$ is replaced by $A(z) \in M_{N}(\boldsymbol{C}\{z\})$. The proof is done by showing that a multiplication by matrix functions with higher order zeros becomes a compact operator on a suitable Banach space which is consisted of $G_{\mu}^{s}(w)$ 's (cf. Lemma 5.5 and Proposition 5.6).

The most fundamental facts are the following that for a monomial $z^{j} D^{k}$ we have

- $z^{j} D^{k}: G_{\kappa}^{(s)}(w) \rightarrow G_{\kappa+\mu_{s}\left(z^{j} D^{k}\right)}^{(s)}(w)$ is bounded.

- $z^{j} D^{k}: G_{\kappa}^{(s)}(w) \rightarrow G_{\kappa+\mu_{s}\left(z^{j} D^{k}\right)+\varepsilon}^{(s)}(w)$ is compact for all $\varepsilon>0$, where $\kappa \geq 0$ is taken so that $\kappa+\mu_{s}\left(x^{i} D^{k}\right) \geq 0$. (For the proof, see [M-Y2].)

In order to fix the image, let us consider a single operator with holomorphic coefficients,

$$
p(z, D)=\sum_{j=0}^{m} a_{j}(z) D^{m-j}, \quad a_{j}(z) \in C\{z\}, a_{0}(z) \not \equiv 0 .
$$

The $s$-Gevrey order $\mu_{s}(p)$ and the $s$-Gevrey principal part $p_{s}(z, D) \in W(\boldsymbol{C})$ or the symbol $p_{s}(z, \zeta) \in \boldsymbol{C}[z, \zeta]$ is similarly defined with (5.4) and (5.5) by taking the Taylor expansion of the coefficients.

By Proposition 5.6 below, which shows the boundedness of the multiplication by holomorphic functions on $G_{\mu}^{(s)}(w)$, we have that

- $p_{s}(z ; D): G_{\kappa}^{(s)}(w) \rightarrow G_{\kappa+\mu_{s}(p)}^{(s)}(w)$ is a bounded mapping.

- $p(z, D)-p_{s}(z ; D): G_{\kappa}^{(s)}(w) \rightarrow G_{\kappa+\mu_{s}(p)}^{(s)}(w)$ is a compact mapping.

Therefore, the study Fredholmness for a single operator $p(z, D)$ on formal $s$-Gevrey space $(s>1)$ is reduced to that for $p_{s}(z, D) \in W(\boldsymbol{C})$. (cf. [M-Y2, Prop. 2].) 
Let us return to the system case $L=(p, A(z))$. In what follows, when we apply Theorem $\mathrm{A}_{\delta}$, we assume $P_{\delta}(z) \in G L_{N}(\boldsymbol{C}[[z]])$. In fact, whenever $P_{\delta}(z)$ has pole singularity, we know the method of handling as in the 3rd step.

As was done in the above steps, by applying Theorem $\mathrm{A}_{\delta}$, we may assume that the system $L$ is already reduced into the following form by a polynomially invertible matrix,

$$
L=\left(p,\left(A_{i j}(z)\right)_{i, j=1, \ldots, k+1}\right), \quad O\left(A_{i j}\right) \geq N p(i \neq j),
$$

with each diagonal subsystem $L_{i}=\left(p, A_{i i}(z)\right)$ satisfying the same property with $\left(p, A_{i}(z)\right)$ in the irreducible decomposition in Theorem $\mathrm{A}_{\delta}$.

Let $T^{(i)}=\left\{t_{j}^{(i)}\right\}_{j=1}^{N_{i}}$ be the $\mathrm{V}$-numbers for $A_{i i}(z)$ associated with $V\left(A_{i i}\right)$ $\left(=V\left(A_{i}\right)\right)$. Let $L_{i}(z, D)=\left(p, A_{i i}(z)\right)$, and put

$$
L_{i}(z, D)=\left(\ell_{j k}^{(i)}(z, D)\right)_{j, k=1, \ldots, N_{i}} .
$$

Let us define

$$
\sigma_{s}\left(L_{i}\right)=\max \left\{(1-s) p+1,(1-s) V\left(A_{i}\right)\right\}
$$

Then it holds that $\mu_{s}\left(L_{i}\right)=N \sigma_{s}\left(L_{i}\right)$ and

$$
\mu_{s}\left(\ell_{j k}^{(i)}\right) \leq(1-s) t_{j}^{(i)}-(1-s) t_{k}^{(i)}+\sigma_{s}\left(L_{i}\right)
$$

Let us define a Banach space $\mathbf{G}_{\mu+(1-s) T^{(i)}}^{(s)}(w)$ by

$$
\mathbf{G}_{\mu+(1-s) T^{(i)}}^{(s)}(w):=\prod_{k=1}^{N_{i}} G_{\mu+(1-s) t_{k}^{(i)}}^{(s)}(w)
$$

Then

$$
L_{i}: \mathbf{G}_{\mu+(1-s) T^{(i)}}^{(s)}(w) \rightarrow \mathbf{G}_{\mu+(1-s) T^{(i)}+\sigma_{s}\left(L_{i}\right)}^{(s)}(w)
$$

becomes a bounded mapping. Therefore

$$
\bigoplus_{i=1}^{k+1} L_{i}: \bigoplus_{i=1}^{k+1} \mathbf{G}_{\mu+(1-s) T^{(i)}}^{(s)}(w) \rightarrow \bigoplus_{i=1}^{k+1} \mathbf{G}_{\mu+(1-s) T^{(i)}+\sigma_{s}\left(L_{i}\right)}^{(s)}(w)
$$

becomes a bounded mapping.

Now in order to show the compactness of the multiple operator by a matrix function of higher order, we give the following rule of taking the set of V-numbers $\left\{T^{(i)}\right\}_{i=1}^{k+1}$.

$$
t_{N_{i}}^{(i)}=t_{1}^{(i+1)}, \quad i=1,2, \ldots, k
$$


The possibility of this choice is guaranteed from that the V-numbers are translation free. By this choice we see that for $i \leq j$ we have

$$
\begin{aligned}
\left|t_{k}^{(i)}-t_{\ell}^{(j)}\right| & \leq\left|t_{k}^{(i)}-t_{N_{i}}^{(i)}\right|+\left|t_{1}^{(i+1)}-t_{N_{i+1}}^{(i+1)}\right|+\cdots+\left|t_{1}^{(j)}-t_{\ell}^{(j)}\right| \\
& \leq\left(N_{i}-1\right) V\left(A_{i}\right)+\left(N_{i+1}-1\right) V\left(A_{i+1}\right)+\cdots+\left(N_{j}-1\right) V\left(A_{j}\right),
\end{aligned}
$$

which follows from the span condition Lemma 3.4. Note that the condition $i \leq j$ is only expedient and is removable.

Now the possibility of truncating the higher order part in the Taylor expansion of the coefficient matrix in $M_{N}(C\{z\})$ is guaranteed by the following lemma.

Lemma 5.5 (compactness of multiplication operator). Let $B(z)=\left(b_{i j}(z)\right) \in$ $M_{N}(C\{z\})$ satisfy $O(B) \geq N p$. Then the following mapping is compact.

$$
B(z): \bigoplus_{i=1}^{k+1} \mathbf{G}_{\mu+(1-s) T^{(i)}}^{(s)}(w) \rightarrow \bigoplus_{i=1}^{k+1} \mathbf{G}_{\mu+(1-s) T^{(i)}+\sigma_{s}\left(L_{i}\right)}^{(s)}(w) .
$$

Proof. We write $B(z)=\left(B^{i j}(z)\right)_{i, j=1}^{k+1}$ by $B^{i j}(z) \in M_{N_{i} \times N_{j}}(C\{z\})$, and $B^{i j}(z)$ $=\left(b_{k, \ell}^{i j}(z)\right)$, where $O\left(b_{k, \ell}^{i j}\right) \geq N p$. For the proof, it is enough to prove that the mapping,

$$
b_{k, \ell}^{i j}(z): G_{\mu+(1-s) t_{\ell}^{(j)}}^{(s)} \rightarrow G_{\mu+(1-s) t_{k}^{(i)}+(1-s) V\left(A_{i}\right)}^{(s)}(w)
$$

is compact, since $(1-s) V\left(A_{i}\right) \leq \sigma_{s}\left(L_{i}\right)$. By the assumption that $O\left(b_{k, \ell}^{i j}\right) \geq N p$ we have

$$
b_{k, \ell}^{i j}(z): G_{\mu+(1-s) t_{\ell}^{(j)}}^{(s)} \rightarrow G_{\mu+(1-s) t_{\ell}^{(j)}+(1-s) N p}^{(s)}(w)
$$

is bounded. Hence, the desired property is proved by showing

$$
\begin{aligned}
& (1-s) t_{\ell}^{(j)}+(1-s) N p<(1-s) t_{k}^{(i)}+(1-s) V\left(A_{i}\right) \quad \text { or } \\
& V\left(A_{i}\right)+t_{k}^{(i)}-t_{\ell}^{(j)}<N p .
\end{aligned}
$$

This is obtained as follows. Let assume $i \leq j$ without loss of generality. Then by (5.24) we have

$$
V\left(A_{i}\right)+t_{k}^{(i)}-t_{\ell}^{(j)} \leq V\left(A_{i}\right)+\left(N_{i}-1\right) V\left(A_{i}\right)+\cdots+\left(N_{j}-1\right) V\left(A_{j}\right)<N p,
$$

since $\sum_{j=1}^{k+1} N_{j}=N, V\left(A_{i}\right)<p(1 \leq i \leq k)$ and $V\left(A_{k+1}\right)=p$.

Now our final aim is to prove the following boundedness property. A similar property was proved in [M-I, A.1], but it was only for small $w>0$ which is insufficient for our purpose. 
Proposition 5.6 (boundedness by multiplication operator). Let $a(z)=$ $\sum_{k=0}^{\infty} a_{k} z^{k} / k ! \in C\{z\}\left(\subset G_{\mu}^{(s)}(w), \forall s>1, \forall \mu \geq 0, \forall w>0\right)$. Then the multiplication by a(z) on $G_{\mu}^{(s)}(w)$ defines a bounded operator with an operator norm,

$$
C(s, \mu) \times\|a\|_{w ; 0}^{(s)} \quad\left(\|a\|_{w ; 0}^{(s)}=\sum_{k=0}^{\infty}\left|a_{k}\right| \frac{\left(s^{s} w\right)^{k}}{(s k) !}<\infty\right)
$$

by a positive constant $C(s, \mu)$ depending only on $s$ and $\mu$.

Proof. Let $a(z) u(z)=v(z)=\sum_{n=0}^{\infty} v_{n} z^{n} / n !, \quad$ where $\quad v_{n}=\sum_{k=0}^{n} a_{k} u_{n-k} \times$ $n ! / k !(n-k) ! . \quad$ This implies

$$
\begin{aligned}
\|v\|_{w ; \mu}^{(s)} & =\sum_{n=0}^{\infty}\left|v_{n}\right| \frac{\left(s^{s} w\right)^{n}}{(s n+\mu) !} \\
& \leq \sum_{n=0}^{\infty}\left\{\sum_{k=0}^{n} \frac{\left|a_{k}\right|}{(s k) !} \frac{\left|u_{n-k}\right|}{(s(n-k)+\mu) !}\left(s^{s} w\right)^{n} \times \frac{(s k) !(s(n-k)+\mu) ! n !}{(s n+\mu) ! k !(n-k) !}\right\} .
\end{aligned}
$$

Therefore the norm estimate (5.27) is obtained by showing the following inequality.

$$
\frac{(s k) !(s(n-k)+\mu) ! n !}{(s n+\mu) ! k !(n-k) !} \leq C(s, \mu),
$$

which is proved in the lemma below.

Lemma 5.7. Let $s>1$ and $\mu \geq 0$. Then, for all $0 \leq k \leq n$, we have

$$
\frac{n !}{k ! \times(n-k) !} \times \frac{(s k) ! \times\{s(n-k)+\mu\} !}{(s n+\mu) !} \leq C(s, \mu) .
$$

by a positive constant $C(s, \mu)$ depending only on $s$ and $\mu$.

Proof. By the Stirling formula, $x !=\Gamma(x+1) \sim c x^{x+1 / 2} / e^{x}(\exists c>0)$, we see that it is sufficient to prove, by a positive constant $C$, that

$$
\begin{aligned}
\frac{n^{n}}{k^{k}(n-k)^{n-k}} & \leq C \times \frac{(s n+\mu)^{s n+\mu}}{(s k)^{s k}\{s(n-k)+\mu\}^{s(n-k)+\mu}} \\
& =C \times \frac{(n+\mu / s)^{s n+\mu}}{(k)^{s k}\{(n-k)+\mu / s\}^{s(n-k)+\mu}} .
\end{aligned}
$$

Here we remark that $n^{n} /\left\{k^{k}(n-k)^{n-k}\right\} \geq 1$. Since $s>1$, we have

$$
\frac{n^{n}}{k^{k}(n-k)^{n-k}} \leq \frac{n^{s n}}{k^{s k}(n-k)^{s(n-k)}} \text {. }
$$


This proves the inequality (5.30) in case $\mu=0$. We notice that

$$
\frac{(n+\mu / s)^{\mu}}{\{(n-k)+\mu / s\}^{\mu}} \geq 1, \quad \mu>0 .
$$

Therefore, by (5.31), we know that for the proof of (5.30) it is sufficient to prove the following inequality,

$$
\frac{n^{s n}}{(n-k)^{s(n-k)}} \leq C \frac{(n+\mu / s)^{s n}}{\{(n-k)+\mu / s\}^{s(n-k)}},
$$

which is equivalent with the following inequality,

$$
\left\{1+\frac{\mu}{s(n-k)}\right\}^{s(n-k)} \leq C\left\{1+\frac{\mu}{s n}\right\}^{s n} .
$$

The sequences on the both hand sides converge to a common $e^{\mu}$ when $n-k$ and $n$ tend to $\infty$. This shows the existence of a positive constant $C$ as above.

\section{Equivalence relation with single operators}

\subsection{Equivalence between system and single operator}

Let a singular system $M(z, D)=(0, A(z))$ be given by

$$
M=z D-A(z), \quad A(z) \in M_{N}(\mathscr{R}[[z]]) .
$$

Then we know that $M(z, D)$ is equivalent with a single operator of the form $M_{\mathrm{sgl}}(z, z D)=(z D)^{N}-\sum_{j=1}^{N} b_{j}(z)(z D)^{N-j}$ with $b_{j}(z) \in \mathscr{R}[[z]]$ by using the cyclic vector of P. Deligne [Del] (cf. [Hs-Si, XIII-7]). Exactly saying, there exists a transformation matrix $F(z) \in G L_{N}(\mathscr{R}[z])$ such that

$$
F: M \mapsto M_{F}=z D-\left[\begin{array}{ccccc}
0 & 1 & 0 & \cdots & 0 \\
0 & 0 & 1 & \cdots & 0 \\
\vdots & \ddots & \ddots & \ddots & \vdots \\
0 & \cdots & 0 & 0 & 1 \\
b_{N}(z) & b_{N-1}(z) & \cdots & b_{2}(z) & b_{1}(z)
\end{array}\right],
$$

The right hand side is nothing but the systemization of a single operator

$$
M_{\mathrm{sgl}}(z, \delta)=\delta^{N}-\sum_{j=1}^{N} b_{j}(z) \delta^{N-j}, \quad \delta=z D .
$$


We shall show such an equivalent relation between a singular system $L=(p, A(z))$ and a single operator which is corresponding to the irreducible decomposition in Theorem $\mathrm{A}_{\delta}$.

For this purpose we need to prepare some notations. We set

$$
\delta_{p}=z^{p+1} D, \quad \delta_{p}(0):=1, \quad \delta_{p}(j):=\delta_{p}-(j-1) p z^{p}(j \geq 1) .
$$

Therefore, $\delta_{0}(j)=\delta$ for $\forall j \geq 1$.

Definition 6.1 (equivalence relation). An equivalence relation " $\sim$ " between a system $M\left(z, \delta_{p}\right)$ and a single operator $M_{\mathrm{sgl}}\left(z, \delta_{p}\right)$ is defined by

$$
\begin{aligned}
M\left(z, \delta_{p}\right) & =\left[\begin{array}{ccccc}
\delta_{p}(1) & -1 & 0 & \ldots & 0 \\
0 & \delta_{p}(2) & -1 & \ldots & 0 \\
\vdots & \ddots & \ddots & \ddots & \vdots \\
0 & \ldots & 0 & \delta_{p}(N-1) & -1 \\
-a_{N}(z) & -a_{N-1}(z) & \ldots & -a_{N-1}(z) & \delta_{p}(N)-a_{1}(z)
\end{array}\right] \\
& \sim \delta_{p}(N) \ldots \delta_{p}(1)-\sum_{j=1}^{N} a_{j}(z) \delta_{p}(N-j) \ldots \delta_{p}(1)=: M_{\mathrm{sgl}}\left(z, \delta_{p}\right) .
\end{aligned}
$$

If a system $L=(p, A(z))$ is reduced into $M\left(z, \delta_{p}\right)$ by a transformation matrix $P(z) \in G L_{N}(\mathscr{R}[[z]])$, that is, $L_{P}=M\left(z, \delta_{p}\right)$, then we also say that $L$ is equivalent with $M_{\mathrm{sgl}}\left(z, \delta_{p}\right)$ which is denoted by $L(z, D) \sim M_{\mathrm{sgl}}\left(z, \delta_{p}\right)$ again.

Note that by an easy calculation we have

$$
\delta_{p}(j) \ldots \delta_{p}(1)=\left(\delta_{p}\right)^{j}+\sum_{k=1}^{j-1} c_{j} p^{k} z^{k p}\left(\delta_{p}\right)^{j-k}, \quad\left\{c_{j}\right\} \subset \boldsymbol{Z} .
$$

Let

$$
\min _{1 \leq j \leq N}\left\{O\left(a_{j}\right) / j\right\}=q \in \boldsymbol{Z} / r \quad(\exists r \in \boldsymbol{N}) .
$$

Then $a_{j}(z)=z^{j q} \tilde{a}_{j}\left(z^{1 / r}\right)$ with $\tilde{a}_{j}(x) \in \boldsymbol{C}[[x]]$ and $\tilde{a}_{j}(0) \neq 0 \quad(\exists j)$. The operator $M_{\mathrm{sgl}}\left(z, \delta_{p}\right)$ is written in the form

$$
M_{\mathrm{sgl}}\left(z, \delta_{p}\right)=\delta_{p}(N) \ldots \delta_{p}(1)-\sum_{j=1}^{N} \tilde{a}_{j}\left(z^{1 / r}\right) z^{j q} \delta_{p}(N-j) \ldots \delta_{p}(1) .
$$

This shows that $M_{\mathrm{sgl}}\left(z, \delta_{p}\right)$ is regular singular at $z=0$ if and only if $q \geq p$. When $q<p$, we have $\rho\left(M_{\mathrm{sgl}}\right)=p-q$ or the steepest slope of the Newton polygon $\mathrm{N}\left(M_{\mathrm{sgl}}\right)$ is $p-q$. 
First, we show the equivalence relation of an irreducible system with a single operator.

Lemma 6.2 (equivalent relation for irreducible system). Let $L=(p, A(z))$ $\left(A(z) \in M_{N}(\mathscr{R}[[z]])\right.$ be an irreducible system. Then we have;

(i) If $L$ is a first kind system in $V$-sense, that is $V(A) \geq p$, then we have

$$
L \sim \delta_{p}(N) \ldots \delta_{p}(1)-\sum_{j=1}^{N} \tilde{a}_{j}(z) z^{j p} \delta_{p}(N-j) \ldots \delta_{p}(1), \quad \tilde{a}_{j}(z) \in \boldsymbol{C}[[z]] .
$$

(ii) If $L$ is a full rank system of irregular singular type with $V(A)=$ $q<p$. Then we have

$$
\begin{aligned}
L & \sim \delta_{p}(N) \ldots \delta_{p}(1)-\sum_{j=1}^{N} z^{j q} \tilde{a}_{j}\left(z^{1 / N}\right) \delta_{p}(N-j) \ldots \delta_{p}(1) \\
& =\delta_{p}^{N}-\sum_{j=1}^{N}\left\{z^{j q} \tilde{a}_{j}\left(z^{1 / N}\right)+o\left(z^{j q}\right)\right\} \delta_{p}^{N-j}, \quad \tilde{a}_{j}(x) \in C[[x]],
\end{aligned}
$$

and for the characteristic polynomial of the principal matrix $\stackrel{\circ}{A}_{T}$ we have

$$
\operatorname{det}\left(\zeta I_{N}-\stackrel{\circ}{A}_{T}\right)=\zeta^{N}-\sum_{j=1}^{N} \tilde{a}_{j}(0) \zeta^{N-j}, \quad \tilde{a}_{N}(0) \neq 0 .
$$

For the convenient sake, the single operator on the right hand sides of (6.6) or (6.7) is denoted by $L_{\mathrm{sgl}}\left(z, \delta_{p}\right)$.

Once we establish this lemma, we immediately obtain the following equivalence relation between a singular system and a single operator.

Theorem 6.3 (equivalence relation for systems). For a given system $L=$ $(p, A(z))\left(A(z) \in M_{N}(\mathscr{R}[[z]])\right)$, let a transformation matrix into the irreducible decomposition be $P_{\delta}(z) \in G L_{N}(\mathscr{R}[[z]])$ in Theorem $A_{\delta}$,

$$
\begin{aligned}
& L_{P_{\delta}}=\bigoplus_{j=1}^{k+1} L_{j}(z, D), \quad L_{j}=\left(p, A_{j}(z)\right), \\
& V\left(A_{1}\right)<\cdots V\left(A_{k}\right)<p \leq V\left(A_{k+1}\right) .
\end{aligned}
$$

Then we have the following equivalent relation

$$
L=(p, A(z)) \sim \bigoplus_{j=1}^{k+1}\left(L_{j}\right)_{\mathrm{sgl}}\left(z, \delta_{p}\right)
$$


Proof of Lemma 6.2. We shall prove the lemma in case (ii), since the case (i) is proved similarly but there is one difference which will be pointed out after the proof of (ii).

From the condition for $L=(p, A(z))$ we can take $V(A)=q \in \boldsymbol{Z} / N(q<p)$ and the associated V-numbers are taken so that $T=\left\{t_{i}=m_{i} / N\right\}_{i=1}^{N} \subset N / N$. Then

$$
z^{T}: L \mapsto L_{z^{T}}=z^{p+1} D-B\left(z^{1 / N}\right), \quad B\left(z^{1 / N}\right) \in M_{N}\left(\mathscr{R}\left[\left[z^{1 / N}\right]\right],\right.
$$

where $\boldsymbol{B}\left(z^{1 / N}\right)=z^{q} \tilde{\boldsymbol{B}}\left(z^{1 / N}\right) \quad\left(\tilde{\boldsymbol{B}}\left(z^{1 / N}\right) \in M_{N}\left(\boldsymbol{C}\left[\left[z^{1 / N}\right]\right]\right)\right.$ with $\operatorname{det} \tilde{\boldsymbol{B}}(0) \neq 0$. For a system $z^{-p} L_{z^{T}}=z D-z^{-p+q} \tilde{B}\left(z^{1 / N}\right)$, by applying the theory of cyclic vector of P. Deligne (cf. [Hs-Si, XIII-7]), we find a transformation matrix $P\left(z^{1 / N}\right) \in$ $G L_{N}\left(\mathscr{R}\left[z^{1 / N}\right]\right)$ such that

$$
\begin{aligned}
& P\left(z^{1 / N}\right): z^{-p} L_{z^{T}} \\
& \mapsto z D-\left[\begin{array}{ccccc}
0 & 1 & 0 & \cdots & 0 \\
0 & 0 & 1 & \cdots & 0 \\
\vdots & \ddots & \ddots & \ddots & \vdots \\
0 & \cdots & \cdots & 0 & 1 \\
a_{N}\left(z^{1 / N}\right) & a_{N-1}\left(z^{1 / N}\right) & \cdots & a_{2}\left(z^{1 / N}\right) & a_{1}\left(z^{1 / N}\right)
\end{array}\right],
\end{aligned}
$$

where $a_{j}\left(z^{1 / N}\right) \in \mathscr{R}\left[\left[z^{1 / N}\right]\right]$. The system on the right hand side is equivalent with a single operator $\delta^{N}-\sum_{j=1}^{N} a_{j}\left(z^{1 / N}\right) \delta^{N-j}(\delta=z D)$.

Here we have to mention that all the systems above and the associated single operator have the common irregularity $p-q$. This implies that $O\left(a_{j}\left(z^{1 / N}\right)\right) \geq(q-p) j \quad(1 \leq j \leq N)$, that is, $a_{j}\left(z^{1 / N}\right)=z^{(q-p) j} \tilde{a}_{j}\left(z^{1 / N}\right)$ with $\tilde{a}_{j}\left(z^{1 / N}\right) \in \boldsymbol{C}\left[\left[z^{1 / N}\right]\right]$.

Moreover, we have the following relation between characteristic polynomials,

$$
\zeta^{N}-\sum_{j=1}^{N} \tilde{a}_{j}(0) \zeta^{N-j}=\operatorname{det}\left(\zeta I_{N}-\stackrel{\circ}{A}_{T}\right), \quad \operatorname{det} \stackrel{\circ}{A}_{T} \neq 0 .
$$

This follows by looking at the fundamental matrix solutions. For the fundamental matrix solutions of these systems, they have a common determining factor $\Lambda(z)$ of diagonal matrix with entries of polynomials of a fractional power $z^{-1 / r}(\exists r \in N)$. From the results in Subsection 4.4, we know that the leading term of $\Lambda(z)$ is given by

$$
\operatorname{Diag}\left[\frac{\alpha_{1}}{q-p} z^{q-p}, \ldots, \frac{\alpha_{N}}{q-p} z^{q-p}\right] .
$$


by using the eigenvalues $\left\{\alpha_{j}\right\}_{j=1}^{N} \in \boldsymbol{C} \backslash\{0\}$ of these characteristic polynomials which proves (6.13) (cf. (4.42)).

From (6.11) and (6.12), we get the following equivalence relation.

$$
\begin{aligned}
z^{T} P\left(z^{1 / N}\right): L \mapsto z^{p}\left\{z^{-p} L_{z^{T}}\right\}_{P\left(z^{1 / N}\right)} \\
=z^{p+1} D-\left[\begin{array}{ccccc}
0 & z^{p} & 0 & \cdots & 0 \\
0 & 0 & z^{p} & \cdots & 0 \\
\vdots & \ddots & \ddots & \ddots & \vdots \\
0 & \cdots & \cdots & 0 & z^{p} \\
a_{N}\left(z^{1 / N}\right) z^{p} & \cdots & \cdots & a_{2}\left(z^{1 / N}\right) z^{p} & a_{1}\left(z^{1 / N}\right) z^{p}
\end{array}\right] .
\end{aligned}
$$

We define

$$
D_{p}(z)=\operatorname{Diag}\left[1, z^{-p}, \ldots, z^{-(N-1) p}\right] .
$$

Then by the transformation matrix $z^{T} P\left(z^{1 / N}\right) D_{p}(z)$ the system $L=(p, A(z))$ is reduced into

$$
L_{z^{T} P\left(z^{1 / N}\right) D_{p}(z)}=\left[\begin{array}{ccccc}
\delta_{p}(1) & -1 & 0 & \cdots & 0 \\
0 & \delta_{p}(2) & -1 & \cdots & 0 \\
\vdots & \ddots & \ddots & \ddots & \vdots \\
0 & \cdots & \cdots & \delta_{p}(N-1) & -1 \\
-a_{N}\left(z^{1 / N}\right) z^{N p} & \cdots & \cdots & -a_{2}\left(z^{1 / N}\right) z^{2 p} & \delta_{p}(N)-a_{1}\left(z^{1 / N}\right) z^{p}
\end{array}\right]
$$

where $a_{j}\left(z^{1 / N}\right) z^{j p}=z^{j q} \tilde{a}_{j}\left(z^{1 / N}\right)$. Indeed, it is sufficient to notice that

$$
z^{(j-1) p}\left(z^{p+1} \frac{d}{d z}\right)\left\{z^{-(j-1) p}\right\}=z^{p+1} \frac{d}{d z}-(j-1) p z^{p}=\delta_{p}(j) .
$$

This completes the proof of (ii).

For the proof of (i), it is enough to notice that we take the $\mathrm{V}$-numbers $T=\left\{t_{i}\right\} \subset N$ which is corresponding to $p$ instead of $V(A)(\geq p)$.

\subsection{Systemization of single operator}

In this subsection, we shall prove that a systemization $P_{\text {sys }}(z, D)$ of a single operator $P(z, D)$ (cf. (6.20)) is equivalent with a decomposition by single operators which correspond to the sides of the Newton polygon $\mathrm{N}\left(P_{\text {sys }}\right)$, i.e, the irreducible subsystems of the decomposition of $P_{\text {sys }}$ in Theorem $\mathrm{A}_{\delta}$. The result says that for each slope of the Newton polygon $\mathrm{N}(P)$ there exists a corresponding single operator. 
Let a single operator $P=P(z, D)$ be given by

$$
P=z^{p}(z D)^{N}+\sum_{i=0}^{N-1} z^{r_{i}} a_{i}(z)(z D)^{i}, \quad a_{i}(z) \in C[[z]], r_{i} \in N
$$

where it is assumed that $a_{i}(0) \neq 0$ whenever $a_{i}(z) \not \equiv 0$. Recall that the Newton polygon $\mathrm{N}(P)$ is a figure defined by

$$
\mathrm{N}(P)=\mathrm{Ch}\left[Q(N, p) \cup \bigcup_{0 \leq i \leq N-1} Q\left(i, r_{i}\right)\right],
$$

where $Q(i, j)=\left\{(x, y) \in \boldsymbol{R}^{2} ; x \leq i, y \geq j\right\}$. Our interest is in the irregular singular case, and we assume that $p>\min _{0 \leq i \leq N-1} r_{i}$. Let the vertexes of $\mathrm{N}(P)$ be given by $\left\{\left(m_{i}, p_{i}\right)\right\}_{i=0}^{k}(k \geq 1)$ so that

$$
N=m_{0}>m_{1}>\cdots>m_{k} \geq 0, \quad p=p_{0}>p_{1}>\cdots>p_{k} \geq 0 .
$$

We put $N_{i}=m_{i-1}-m_{i}$ for $1 \leq i \leq k+1 \quad\left(m_{k+1}:=0\right)$. Then we have a decomposition $N=\sum_{i=1}^{k+1} N_{i}$. Let $\left\{\rho_{i}\right\}_{i=0}^{k+1}$ be the slopes of sides of $\mathrm{N}(P)$,

$$
\begin{aligned}
& \infty=\rho_{0}>\rho_{1}>\cdots>\rho_{k}>\rho_{k+1}=0, \\
& \rho_{i}=\frac{p_{i-1}-p_{i}}{m_{i-1}-m_{i}}=\frac{p_{i-1}-p_{i}}{N_{i}} \quad(1 \leq i \leq k) .
\end{aligned}
$$

Then we have $m_{i}=N-\sum_{j=1}^{i} N_{j}\left(m_{0}=N\right), p_{i}=p-\sum_{j=1}^{i} \rho_{j} N_{j}\left(p_{0}=p\right)$ and

$$
\left\{\begin{array}{l}
r_{m_{i}}=p_{i}, \quad a_{m_{i}}(0) \neq 0 \quad(0 \leq i \leq k) \\
m_{i} \leq j \leq m_{i-1} \Rightarrow r_{j} \geq p-\sum_{\ell=1}^{i-1} \rho_{\ell} N_{\ell}-\left(m_{i-1}-j\right) \rho_{i} \\
\quad=p_{i-1}-\left(m_{i-1}-j\right) \rho_{i}
\end{array}\right.
$$

For the convenience sake we rewrite the coefficients $\left\{z^{r_{i}} a_{i}(z)\right\}$ as follows.

$$
\left\{\begin{array}{l}
m_{i} \leq j \leq m_{i-1} \Rightarrow r_{i}=p-\sum_{j=1}^{i-1} \rho_{j} N_{j}-\left(m_{i-1}-j\right) \rho_{i} \\
a_{i}(0)=0 \text { if } r_{i}>p_{i-1}-\left(m_{i-1}-j\right) \rho_{i} \text { in }(6.18) .
\end{array}\right.
$$

Now we introduce a systemization $P_{\text {sys }}(z, D)$ of the single operator $P(z, D)$ as follows.

Let $P u=0$ be the associated homogeneous equation. We introduce an $N$-vector function $U(z)$ by

$$
U(z)={ }^{\mathrm{t}}\left(u_{1}(z), \ldots, u_{N}(z)\right), \quad u_{j}(z):=\left(z D_{z}\right)^{N-j} u(z) .
$$


Then the corresponding system for $U(z), P_{\text {sys }} U(z)=0$, is given by

$$
P_{\mathrm{sys}}(z, D):=z^{p+1} D-\left[\begin{array}{ccccc}
-z^{r_{N-1}} a_{N-1}(z) & \cdots & \cdots & -z^{r_{1}} a_{1}(z) & -z^{r_{0}} a_{0}(z) \\
z^{p} & 0 & \cdots & 0 & 0 \\
\vdots & \ddots & \ddots & \vdots & \vdots \\
0 & \cdots & z^{p} & 0 & 0 \\
0 & \cdots & 0 & z^{p} & 0
\end{array}\right]
$$

which we call the systemization of the single operator $P$.

Theorem 6.4 (decomposition of single operator). Let a single operator $P(z, D)=z^{p}(z D)^{N}+\sum_{i=0}^{N-1} z^{r_{i}} a_{i}(z)(z D)^{i}$ be as above. Then for the systemization $P_{\text {sys }}(z, D)$ we have the following equivalence relation.

$$
P_{\text {sys }}(z, D) \sim \bigoplus_{j=1}^{k+1} P_{j}\left(z, \delta_{p}\right)
$$

which satisfy the following conditions; For $1 \leq j \leq k, P_{j}$ is an operator of irregularity $\rho\left(P_{j}\right)=\rho_{j}$ written in the form,

$$
P_{j}\left(z, \delta_{p}\right)=\delta_{p}^{N_{j}}+\sum_{\ell=0}^{N_{j}-1}\left\{b_{\ell}^{(j)} z^{\left(N_{j}-\ell\right)\left(p-\rho_{j}\right)}+o\left(z^{\left(N_{j}-\ell\right)\left(p-\rho_{j}\right)}\right)\right\} \delta_{p}^{\ell},
$$

with

$$
b_{\ell}^{(j)}=\frac{a_{m_{j}+\ell}(0)}{a_{m_{j-1}}(0)}, \quad b_{0}^{(j)}=\frac{a_{m_{j}}(0)}{a_{m_{j-1}}(0)} \neq 0, \quad a_{m_{0}}(0):=a_{N}(0)=1,
$$

and $P_{k+1}$ is a first kind singular operator,

$$
P_{k+1}\left(z, \delta_{p}\right)=\delta_{p}^{N_{k+1}}+\sum_{\ell=0}^{N_{k+1}-1} O\left(z^{\left(N_{k+1}-\ell\right) p}\right) \delta_{p}^{\ell}
$$

These show that each $N\left(P_{j}\right)$ has only one nontrivial side of slope $\rho_{j}$ which corresponds to the associated side of $\mathrm{N}\left(P_{\text {sys }}\right)=\mathrm{N}(P)+(0, p(N-1))$.

Proof. The theorem is proved by applying Theorem $\mathrm{A}_{\tau}$ and then Theorem 6.3. This means that the theorem is proved by constructing a transformation matrix $P_{\tau}(z) \in G L_{N}(\boldsymbol{C}[[z]])$ of a lower triangular matrix which reduces the system $P_{\text {sys }}(z, D)$ into a blocked triangular form,

$$
\left(p, \operatorname{Triang}\left[A_{1}(z), \ldots, A_{k}(z), A_{k+1}(z)\right]\right), \quad A_{j}(z) \in M_{N_{j}}(\mathscr{R}[[z]])
$$


with conditions that $V\left(A_{k+1}\right) \geq p$ and for $1 \leq j \leq k$

$$
A_{j}(z)=\left[\begin{array}{cccc}
-b_{N_{j}-1}^{(j)}(z) & -b_{N_{j}-2}^{(j)}(z) & \cdots & -b_{0}^{(j)}(z) \\
z^{p} & 0 & \cdots & 0 \\
& \ddots & \ddots & \vdots \\
0 & \cdots & z^{p} & 0
\end{array}\right], \quad V\left(A_{j}\right)=p-\rho_{j}
$$

which has the principal matrix function (associated with a V-numbers $T^{(j)}$ ),

$$
\left(\AA_{j}\right)_{T^{(j)}}(z)=\left[\begin{array}{cccc}
-b_{N_{j}-1}^{(j)} z^{p-\rho_{j}} & -b_{N_{j}-2^{(j)}} z^{p-2 \rho_{j}} & \cdots & -b_{0}^{(j)} z^{p-N_{j} \rho_{j}} \\
z^{p} & 0 & \cdots & 0 \\
& \ddots & \ddots & \vdots \\
0 & \cdots & z^{p} & 0
\end{array}\right], \quad(\mathrm{cf} .(6.22)) .
$$

In what follows we shall show an outline of proof in case $k=2$ and $N_{3}=0$, since the exact and complete proof is messy and tedious.

For the convenience, we put $N_{1}=m, N_{2}=n(N=m+n), \rho_{1}=\rho$ and $\rho_{2}=\eta(p>\rho>\eta>0)$. We write the matrix $A(z)$ in a blocked form associated with the decomposition $N=m+n$,

$A(z)=\left[\begin{array}{ll}A_{11}(z) & A_{12}(z) \\ A_{21}(z) & A_{22}(z)\end{array}\right]$

$=z^{p} \times\left[\begin{array}{cccccccc}a_{m-1} z^{-\rho} & a_{m-2} z^{-2 \rho} & \cdots & a_{0} z^{-m \rho} & b_{n-1} z^{-m \rho-\eta} & b_{n-2} z^{-m \rho-2 \eta} & \cdots & b_{0} z^{-m \rho-n \eta} \\ 1 & 0 & \cdots & 0 & & & & \\ \vdots & \ddots & \ddots & \vdots & & & O & \\ 0 & \ldots & 1 & 0 & & & & \\ -- & -- & -- & -- & -- & -- & -- & -- \\ 0 & \cdots & 0 & 1 & 0 & 0 & \cdots & 0 \\ & & & & 1 & 0 & \cdots & 0 \\ & & & & \vdots & \ddots & \ddots & \vdots \\ & & & & 0 & \cdots & 1 & 0\end{array}\right]$

where $a_{j}=a_{j}(z), \quad b_{j}=b_{j}(z) \in \boldsymbol{C}[[z]], a_{0}(0) \neq 0$ and $b_{0}(0) \neq 0$. Moreover we have $m \rho, n \eta \in \boldsymbol{N}$.

Noticing that $V(A)=p-\rho$ and $\rho>\eta$ we can take the $\mathrm{V}$-numbers $T=$ $\left\{t_{i}\right\}_{i=1}^{m+n}$ by

$$
t_{i}=i \rho \quad(1 \leq i \leq m), \quad t_{m+j}=m \rho+j \eta \quad(1 \leq j \leq n) .
$$

In fact, 


$$
\begin{aligned}
& t_{1}-t_{j}+V(A)=\rho-j \rho+(p-\rho)=p-j \rho \quad(1 \leq j \leq m), \\
& t_{1}-t_{m+j}+V(A)=\rho-(m \rho+j \eta)+(p-\rho)=p-m \rho-j \eta \quad(1 \leq j \leq n), \\
& t_{i+1}-t_{i}+V(A)=p \quad(1 \leq i \leq m-1), \\
& t_{m+j}-t_{m+j-1}+V(A)=\eta+(p-\rho)=p-(\rho-\eta)<p \quad(1 \leq j \leq n) .
\end{aligned}
$$

This shows that the principal matrix function $\AA_{T}(z)$ is given by

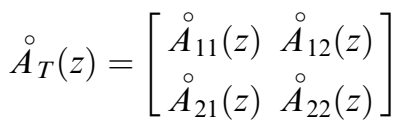

$$
\begin{aligned}
& =z^{p} \times\left[\begin{array}{cccccccc}
\alpha_{m-1} z^{-\rho} & \alpha_{m-2} z^{-2 \rho} & \ldots & \alpha_{0} z^{-m \rho} & \beta_{n-1} z^{-m \rho-\eta} & \beta_{n-2} z^{-m \rho-2 \eta} & \cdots & \beta_{0} z^{-m \rho-n \eta} \\
1 & 0 & \ldots & 0 & & & & \\
\vdots & \ddots & \ddots & \vdots & & & & \\
0 & \cdots & 1 & 0 & & & & \\
-- & -- & -- & -- & -- & -- & -- & -- \\
0 & \cdots & 0 & 0 & 0 & 0 & \cdots & 0 \\
& & & & 0 & 0 & \cdots & 0 \\
& & & & \vdots & \ddots & \ddots & \vdots \\
& & & & 0 & \cdots & 0 & 0
\end{array}\right] \text {, }
\end{aligned}
$$

where $\alpha_{j}=a_{j}(0)\left(\alpha_{0} \neq 0\right)$ and $\beta_{j}=b_{j}(0)\left(\beta_{0} \neq 0\right)$.

Since $\AA_{21}(z)=O$ and $\AA_{22}(z)=O$, we are in the position to start the reduction procedure in Proposition 4.3 to kill (2,1)-block (cf. Remark 4.6-2)).

The first approximation of the transformation matrix is given by

$$
R_{1}(z)=\left[\begin{array}{cc}
I_{m} & O \\
A_{21}(z) \stackrel{\circ}{A_{11}^{-1}(z)} & I_{n}
\end{array}\right] \in G L_{N}(\boldsymbol{C}[z]),
$$

where

$$
\stackrel{\circ}{11}_{11}^{-1}(z)=z^{-p}\left[\begin{array}{ccccc}
0 & 1 & 0 & \cdots & 0 \\
0 & 0 & 1 & \cdots & 0 \\
\vdots & \ddots & \ddots & \ddots & \vdots \\
0 & 0 & \cdots & 0 & 1 \\
\frac{1}{\alpha_{0}} z^{m \rho} & -\frac{\alpha_{m-1}}{\alpha_{0}} z^{(m-1) \rho} & \cdots & -\frac{\alpha_{2}}{\alpha_{0}} z^{2 \rho} & -\frac{\alpha_{1}}{\alpha_{0}} z^{\rho}
\end{array}\right] .
$$

Then by an easy calculation we see that

$$
R_{1}:(p, A(z)) \mapsto(p, B(z)), \quad B(z)=\left[\begin{array}{ll}
B_{11}(z) & B_{12}(z) \\
B_{21}(z) & B_{22}(z)
\end{array}\right]
$$


with $B_{11}(z)=A_{11}(z)+$ negligible matrix, $B_{12}(z)=A_{12}(z)$ and

$$
B_{22}(z)=\left[\begin{array}{cccc}
-\frac{b_{n-1}(z)}{\alpha_{0}} z^{p-\eta} & -\frac{b_{n-2}(z)}{\alpha_{0}} z^{p-2 \eta} & \cdots & -\frac{b_{0}(z)}{\alpha_{0}} z^{p-n \eta} \\
z^{p} & 0 & \cdots & 0 \\
& \ddots & \ddots & \vdots \\
0 & \cdots & z^{p} & 0
\end{array}\right] .
$$

By this expression we know that $\left(p, B_{22}(z)\right)$ is a full rank system of $V\left(B_{22}\right)=p-\eta$, since $b_{0}(0) \neq 0$ by the assumption.

By continuing the reduction in Proposition 4.3, we get the desired blocked triangular system.

Remark 6.5. As we stated at the beginning of the proof, the transformation matrix $P_{\tau}(z)$ is taken in $G L_{N}(C[[z]])$. Indeed, this is understood from the choice of the V-numbers $T=\left\{t_{i}\right\}_{i=1}^{m+n}$ by (6.25) which is increasing as $i$. This shows that the transformation matrix $P_{\delta}(z) \in G L_{N}(\mathscr{R}[[z]])$ in Theorem $\mathrm{A}_{\delta}$ may have pole singularity. This is understood from Proposition 4.4 as follows. In order to kill $(1,2)$-block from the coefficient matrix, we use the upper triangular matrices of the form,

$$
S(z)=\left(\begin{array}{cc}
I_{m} & S_{12}(z) \\
O & I_{n}
\end{array}\right) \in M_{N}^{(T)}(\mathscr{R}[[z]]), \quad V(S)=0,
$$

with $S_{12}(z)=\left(s_{i, m+j}(z)\right) \in M_{m \times n}(\mathscr{R}[[z]])$. The order of zeros of $s_{i, m+j}(z)$ is estimated by

$$
O\left(s_{i, m+j}\right) \geq t_{i}-t_{m+j}=i \rho-(m \rho-j \eta)=-(m-i) \rho-j \eta,
$$

where the right hand side is negative.

Although the transformation matrix $P_{\delta}(z)$ may have pole singularity, but we see that the coefficient matrices of systems which appear in the reduction procedure have no pole singularity. In fact, throughout the procedure for the coefficient matrices of systems the Volevič weight and the V-numbers are commonly taken by $p-\rho$ and $T=\left\{t_{i}\right\}_{i=1}^{m+n}$. Therefore the order of zeros of the $(i, j)$-entry of the coefficient matrix is estimated from below by

$$
t_{i}-t_{j}+(p-\rho) \geq-\{(m-1) \rho+n \eta\}+(p-\rho)=p-(m \rho+n \eta) \geq 0,
$$

which shows the pole singularity never happen. The last inequality follows from the determination of the Newton polygon and the slopes. This proves the assertion.

In case of general $k \geq 2$, we only notice that the $\mathrm{V}$-numbers $T$ of the coefficient matrix is taken similarly with (6.25) and the inequality $p \geq \rho_{1} N_{1}$ $+\cdots+\rho_{k} N_{k}$ holds. 
Example 6.6 (Single operator). We consider

$$
P=z^{3}(z D)^{2}-z(z D)-1 .
$$

The systemization of this operator is given by

$$
P_{\text {sys }}=z^{4} D-A(z), \quad A(z)=\left(\begin{array}{cc}
z & 1 \\
z^{3} & 0
\end{array}\right) .
$$

We kill $(1,2)$-entry $a_{12}(z)=1$ by a matrix

$$
S(z)=\left(\begin{array}{cc}
1 & -z^{-1} \\
0 & 1
\end{array}\right) \notin G L_{2}(C[z])
$$

which has a pole singularity, but

$$
S(z): P_{\text {sys }} \mapsto \tilde{L}=z^{4} D-\tilde{A}(z), \quad \tilde{A}(z)=\left(\begin{array}{cc}
z+z^{2} & -z-z^{2} \\
z^{3} & -z^{2}
\end{array}\right) \in M_{2}(\boldsymbol{C}[z]) .
$$

We kill $-z$ on the $(1,2)$-place by

$$
\left(\begin{array}{ll}
1 & 1 \\
0 & 1
\end{array}\right): \tilde{L} \mapsto z^{4} D-\left(\begin{array}{cc}
z+z^{2}-z^{3} & z^{2}-z^{3} \\
z^{3} & -z^{2}+z^{3}
\end{array}\right) .
$$

This shows that

$$
\mathrm{N}\left(L_{P}\right)=\mathrm{N}\left(z^{4} D-z\right)+\mathrm{N}\left(z^{4} D+z^{2}\right)=\mathrm{N}(P)+(0,3) .
$$

\section{Some numerical experiments}

In this section we give some numerical experiments according to the type of systems in Definition 3.6 and Subsection 4.4. We note that the transformation matrix $R(z)$ in Proposition 4.3, which reduces the system into a blocked triangular form, is not constructed by solving (4.11), but it will be constructed by the method of (4.18), (4.19) in Remark 4.6-2), since it is easier to calculate. The following results are experimented by Wolfram Mathematica 7.

\subsection{Degenerate system $=$ Nilpotent principal matrix}

Let us consider the following system,

$$
L=(3, A(z)), \quad A(z)=\left[\begin{array}{ccc}
z^{2} & 3 & z \\
-z^{4} & -2 z^{2} & z^{4} \\
z^{4} & z & z^{2}
\end{array}\right] .
$$


The Volevič weight $V(A)$ and the associated V-numbers $T=\left\{t_{i}\right\}$ are;

$$
V(A)=2, \quad T=\left\{t_{1}=0, t_{2}=2, t_{3}=1\right\} .
$$

The associated principal matrix $\AA_{T}$ and its eigenvalues $\left\{\alpha_{j}\right\}$ are;

$$
\stackrel{\circ}{A}_{T}=\left[\begin{array}{ccc}
1 & 3 & 1 \\
-1 & -2 & 0 \\
0 & 1 & 1
\end{array}\right], \quad\left\{\alpha_{j}\right\}=\{0,0,0\} .
$$

This shows that the system is of degenerate type, and we have to reduce it into a non degenerate system (cf. Proposition 4.2). We exchange rows and columns in the order of V-numbers, i.e, the second and the third are exchanged. Then the reduced system and its principal matrix functions become;

$$
L \sim\left(3,\left[\begin{array}{ccc}
z^{2} & z & 3 \\
z^{4} & z^{2} & z \\
-z^{4} & z^{4} & -2 z^{2}
\end{array}\right]\right), \quad\left[\begin{array}{ccc}
z^{2} & z & 3 \\
0 & z^{2} & z \\
-z^{4} & 0 & -2 z^{2}
\end{array}\right] .
$$

A left null vector of the principal matrix function is given by $\vec{\ell}(z)=\left(z^{2},-z, 1\right)$. Let a transformation matrix be;

$$
\left[\begin{array}{ccc}
1 & 0 & 0 \\
0 & 1 & 0 \\
-z^{2} & z & 1
\end{array}\right]
$$

Then the system is reduced into the following one with the same Volevič weight and V-numbers,

$$
L \sim\left(3,\left[\begin{array}{ccc}
-2 z^{2} & 4 z & 3 \\
-z^{3}+z^{4} & 2 z^{2} & z \\
z^{5} & 0 & 0
\end{array}\right]\right)
$$

To this system the principal matrix function and its non trivial principal minor are;

$$
\left[\begin{array}{ccc}
-2 z^{2} & 4 z & 3 \\
-z^{3} & 2 z^{2} & z \\
0 & 0 & 0
\end{array}\right], \quad\left[\begin{array}{cc}
-2 z^{2} & 4 z \\
-z^{3} & 2 z^{2}
\end{array}\right]
$$

A left null vector of the principal minor is given by $(-z / 2,1)$. Let a transformation matrix be;

$$
\left[\begin{array}{ccc}
1 & 0 & 0 \\
z / 2 & 1 & 0 \\
0 & 0 & 1
\end{array}\right]
$$


Then the system is reduced into

$$
L \sim\left(3,\left[\begin{array}{ccc}
0 & 4 z & 3 \\
z^{4} / 2 & 0 & -z / 2 \\
z^{5} & 0 & 0
\end{array}\right]\right) \underset{\text { put }}{=}(3, B(z)) .
$$

To this reduced system, the Volevič weight $V(B)$ and the associated V-numbers $T=\left\{t_{i}\right\}$ are;

$$
V(B)=\frac{7}{3}, \quad\left\{t_{1}=0, t_{2}=\frac{4}{3}, t_{3}=\frac{8}{3}\right\}
$$

The principal matrix $\stackrel{\circ}{B}_{T}$ and its eigenvalues are;

$$
\stackrel{\circ}{B}_{T}=\left[\begin{array}{ccc}
0 & 4 & 0 \\
0 & 0 & -1 / 2 \\
1 & 0 & 0
\end{array}\right], \quad\left\{e^{(\pi / 3) i} \sqrt[3]{2},-\sqrt[3]{2}, e^{-(\pi / 3) i} \sqrt[3]{2}\right\}
$$

\section{Conclusion:}

- The reduced system $(3, B(z))$ is a full rank system of $\rho(L)=3-V(B)$ $=2 / 3$ with a principal matrix of distinct eigenvalues. Therefore this is reducible into a diagonal system by a formal transformation matrix of power series of $z^{1 / 3}$. (cf. Theorem $\mathrm{A}_{\delta}$ and Subsection 4.4.)

- The leading term of the determining factor of the fundamental matrix solution is

$$
\left[\begin{array}{ccc}
-\frac{3 \alpha}{2} z^{-2 / 3} & 0 & 0 \\
0 & -\frac{3 \beta}{2} z^{-2 / 3} & 0 \\
0 & 0 & -\frac{3 \gamma}{2} z^{-2 / 3}
\end{array}\right]
$$

where $\{\alpha, \beta, \gamma\}$ are the above eigenvalues.

- The Newton polygon $\mathrm{N}(L)$ is obtained from $\operatorname{det}\left(z^{4} \lambda I_{3}-\stackrel{\circ}{B}_{T}(z)\right)=$ $z^{12} \lambda^{3}+2 z^{7}$. Hence, $\mathrm{N}(L)$ has two vertexes $(0,7)$ and $(3,9)$ with a non trivial side of slope $2 / 3$. The index $\chi\left(L ; \mathscr{G}^{s}\right)$ has only one discontinuous point in $s \geq 1$ at $s=1+3 / 2=5 / 2$.

\subsection{Non degenerate system $=$ Non nilpotent principal matrix}

We consider the following system,

$$
L=(4, A(z)), \quad A(z)=\left[\begin{array}{ccc}
z^{2} & 1 & z^{2} \\
z^{5} & z^{2} & -z^{3} \\
2 z^{3} & z & z^{2}
\end{array}\right]
$$


The V-weight $V(A)$ and the associated V-numbers $T=\left\{t_{i}\right\}$ are;

$$
V(A)=2, \quad T=\left\{t_{1}=0, t_{2}=2, t_{3}=1\right\} .
$$

The associated principal matrix $\AA_{T}$ and its eigenvalues $\left\{\alpha_{j}\right\}$ are;

$$
\stackrel{\circ}{A}_{T}=\left[\begin{array}{ccc}
1 & 1 & 0 \\
0 & 1 & -1 \\
2 & 1 & 1
\end{array}\right], \quad\left\{\alpha_{j}\right\}=\left\{\frac{3 \pm i \sqrt{7}}{2}, 0\right\} .
$$

This shows that the system is of non degenerate type of $\rho(L)=4-V(A)=2$, but it is not of full rank. So we need to reduce the system into a triangular form following Theorem $\mathrm{A}_{\tau}$. We exchange the rows and columns in the order of V-numbers, i.e. the second and the third are exchanged. Then the system and the principal matrix function become;

$$
L \sim\left(4,\left[\begin{array}{ccc}
z^{2} & z^{2} & 1 \\
2 z^{3} & z^{2} & z \\
z^{5} & -z^{3} & z^{2}
\end{array}\right]\right), \quad\left[\begin{array}{ccc}
z^{2} & 0 & 1 \\
2 z^{3} & z^{2} & z \\
0 & -z^{3} & z^{2}
\end{array}\right] .
$$

The left null vector of the principal matrix function is $\left(-2 z^{2}, z, 1\right)$ and let a transformation matrix be;

$$
\left[\begin{array}{ccc}
1 & 0 & 0 \\
0 & 1 & 0 \\
2 z^{2} & -z & 1
\end{array}\right]
$$

Then the reduced system and its principal matrix function are;

$$
L \sim\left(4,\left[\begin{array}{ccc}
3 z^{2} & -z+z^{2} & 1 \\
4 z^{3} & 0 & z \\
z^{5}-4 z^{6} & -2 z^{4}+z^{5} & 0
\end{array}\right]\right), \quad\left[\begin{array}{ccc}
3 z^{2} & -z & 1 \\
4 z^{3} & 0 & z \\
0 & 0 & 0
\end{array}\right] .
$$

In order to kill $\left(z^{5},-2 z^{4}\right)$ on the $\{(3,1),(3,2)\}$ block of the coefficient matrix, we consider the following matrix,

$$
\left[\begin{array}{cc}
3 z^{2} & -z \\
4 z^{3} & 0 \\
z^{5} & -2 z^{4}
\end{array}\right]
$$

The left null vector of this matrix is $\left(-2 z^{3}, 5 z^{2} / 4,1\right)$. Let a transformation matrix be;

$$
\left[\begin{array}{ccc}
1 & 0 & 0 \\
0 & 1 & 0 \\
2 z^{3} & -5 z^{2} / 4 & 1
\end{array}\right]
$$


Then the system is reduced into

$$
\left(4,\left[\begin{array}{ccc}
3 z^{2}+2 z^{3} & -z-z^{2} / 4 & 1 \\
4 z^{3}+2 z^{4} & -5 z^{3} / 4 & z \\
-11 z^{6} / 2-6 z^{7} & -z^{5} / 16+5 z^{6} / 2 & -3 z^{3} / 4
\end{array}\right]\right)
$$

We, further, kill $\left(-(11 / 2) z^{6},-(1 / 16) z^{5}\right)$ on $\{(3,1),(3,2)\}$ block. We consider the following matrix.

$$
\left[\begin{array}{cc}
3 z^{2} & -z \\
4 z^{3} & 0 \\
-11 z^{6} / 2 & -z^{5} / 16
\end{array}\right] .
$$

The left null vector of this matrix is $\left(-(1 / 16) z^{4},(91 / 64) z^{3}, 1\right)$. Let a transformation matrix be;

$$
\left[\begin{array}{ccc}
1 & 0 & 0 \\
0 & 1 & 1 \\
z^{4} / 16 & -91 z^{3} / 64 & 1
\end{array}\right]
$$

Then the system is reduced into

$$
\left(4,\left[\begin{array}{ccc}
3 z^{2}+2 z^{3}+z^{4} / 16 & -z-z^{2} / 4-91 z^{3} / 64 & 1 \\
4 z^{3}+2 z^{4}+z^{5} / 16 & -5 z^{3} / 4-91 z^{4} / 64 & z \\
-213 z^{7} / 64+O\left[z^{8}\right] & 231 z^{6} / 128+O\left[z^{7}\right] & -3 z^{3} / 4+87 z^{4} / 64
\end{array}\right]\right)
$$

By continuing these operations we can kill the $\{(3,1),(3,2)\}$ block from the coefficient matrix, which will be completed Theorem $\mathrm{A}_{\tau}$.

Next, if one wants to get a blocked diagonal form in Theorem $\mathrm{A}_{\delta}$ we have to kill $\{(1,3),(2,3)\}$ block from the coefficient matrix. matrix;

In order to kill ${ }^{\mathrm{t}}(1, z)$ on $\{(1,3),(2,3)\}$ block, we consider the following

$$
\left[\begin{array}{ccc}
3 z^{2} & -z & 1 \\
4 z^{3} & 0 & z
\end{array}\right]
$$

The right null vector of this matrix is given by ${ }^{\mathrm{t}}\left(-1 /\left(4 z^{2}\right), 1 /(4 z), 1\right)$. Let a transformation matrix be;

$$
\left[\begin{array}{ccc}
1 & 0 & -1 /\left(4 z^{2}\right) \\
0 & 1 & 1 /(4 z) \\
0 & 0 & 1
\end{array}\right]
$$


Then the system is reduced into

$$
\left(4,\left[\begin{array}{ccc}
3 z^{2}+2 z^{3}+O\left[z^{4}\right] & -z-z^{2} / 4+O\left[z^{3}\right] & -3 z / 4+O\left[z^{2}\right] \\
4 z^{3}+2 z^{4}+O\left[z^{5}\right] & -5 z^{3} / 4+O\left[z^{4}\right] & -5 z^{2} / 8+O\left[z^{3}\right] \\
O\left[z^{7}\right] & O\left[z^{6}\right] & -3 z^{3} / 4+87 z^{4} / 64+O\left[z^{5}\right]
\end{array}\right]\right)
$$

If one wants to make further reduction, we consider a matrix

$$
\left[\begin{array}{ccc}
3 z^{2} & -z & -3 z / 4 \\
4 z^{3} & 0 & -5 z^{2} / 8
\end{array}\right]
$$

By using the right null vector of this matrix, we can construct a transformation matrix to kill ${ }^{\mathrm{t}}\left(-(3 / 4) z,-(5 / 8) z^{2}\right)$ as above, and finally we obtain the blocked diagonal form.

$$
\left(4,\left[\begin{array}{ccc}
3 z^{2}+O\left[z^{3}\right] & -z+O\left[z^{2}\right] & 0 \\
4 z^{3}+O\left[z^{4}\right] & O\left[z^{3}\right] & 0 \\
0 & 0 & -3 z^{3} / 4+O\left[z^{4}\right]
\end{array}\right]\right)
$$

In order to make the V-numbers flat we we apply the following transformation matrix

$$
\left[\begin{array}{lll}
1 & 0 & 0 \\
0 & z & 0 \\
0 & 0 & 1
\end{array}\right]
$$

and we get the following system

$$
\left(4,\left[\begin{array}{ccc}
3 z^{2}+O\left[z^{3}\right] & -z^{2}+O\left[z^{3}\right] & 0 \\
4 z^{2}+O\left[z^{3}\right] & O\left[z^{3}\right] & 0 \\
0 & 0 & -3 z^{3} / 4+O\left[z^{4}\right]
\end{array}\right]\right)
$$

This shows that the system is reducible into a diagonal system by a transformation of formal power series.

\section{Conclusion:}

- The principal parts in irreducible decomposition in the canonical form are

$$
\left(4,\left[\begin{array}{cc}
3 z^{2} & -z \\
4 z^{3} & 0
\end{array}\right]\right) \quad \text { or } \quad\left(4,\left[\begin{array}{cc}
3 z^{2} & -z^{2} \\
4 z^{2} & 0
\end{array}\right]\right), \quad\left(4,-\frac{3 z^{3}}{4}\right) .
$$

- Leading term of the determining factor of the fundamental matrix solution is; 


$$
\left[\begin{array}{ccc}
-\alpha z^{-2} / 2 & 0 & 0 \\
0 & -\beta z^{-2} / 2 & 0 \\
0 & 0 & 3 z^{-1 / 4}
\end{array}\right], \quad\{\alpha, \beta\}=\left\{\frac{3 \pm i \sqrt{7}}{2}\right\} .
$$

- The Newton polygon $\mathrm{N}(L)$ has three vertexes $\{(0,7),(1,8),(3,12)\}$ and two non trivial sides of slopes 1 and 2 , which is obtained from the characteristic polynomials of the irreducible subsystems in the blocked decomposition. This shows that $\chi\left(L ; \mathscr{G}^{S}\right)$ has two discontinuous points $s=3 / 2$ and 2 .

\subsection{Full rank system when the principal matrix has only one eigenvalue}

The system we consider is

$$
L=(4, A(z)), \quad A(z)=\left[\begin{array}{ccc}
z+z^{2} & z & 1 \\
z^{2} & z-z^{2} & z^{3} \\
z^{4} & z^{3} & z+z^{3}
\end{array}\right] .
$$

The Volevič weight $V(A)$ and the associated V-numbers $T=\left\{t_{i}\right\}$ are;

$$
V(A)=1, \quad T=\left\{t_{1}=0, t_{2}=0, t_{3}=1\right\} .
$$

The principal matrix $\stackrel{\circ}{A}_{T}$ and its eigenvalues $\left\{\alpha_{j}\right\}$ are;

$$
\stackrel{\circ}{A}_{T}=\left[\begin{array}{lll}
1 & 1 & 1 \\
0 & 1 & 0 \\
0 & 0 & 1
\end{array}\right], \quad\left\{\alpha_{j}\right\}=\{1,1,1\} .
$$

We reduced the system by the exponential transformation matrix,

$$
\left[\begin{array}{ccc}
e^{-1 /\left(3 z^{3}\right)} & 0 & 0 \\
0 & e^{-1 /\left(3 z^{3}\right)} & 0 \\
0 & 0 & e^{-1 /\left(3 z^{3}\right)}
\end{array}\right]
$$

Then the reduced system becomes;

$$
\left(4,\left[\begin{array}{ccc}
z^{2} & z & 1 \\
z^{2} & -z^{2} & z^{3} \\
z^{4} & z^{3} & z^{3}
\end{array}\right]\right) \underset{\text { put }}{=}(4, B(z))
$$

The Volevič weight $V(B)$ and the associated V-numbers $T=\left\{t_{i}\right\}$ are;

$$
V(B)=\frac{3}{2}, \quad T=\left\{t_{1}=0, t_{2}=\frac{1}{2}, t_{3}=\frac{3}{2}\right\} .
$$


The principal matrix $\stackrel{\circ}{B}_{T}$ and its eigenvalues $\left\{\alpha_{j}\right\}$ are;

$$
\stackrel{\circ}{B}_{T}=\left[\begin{array}{lll}
0 & 1 & 1 \\
1 & 0 & 0 \\
0 & 0 & 0
\end{array}\right], \quad\left\{\alpha_{j}\right\}=\{-1,1,0\}
$$

The principal matrix function and its principal minor are

$$
\left[\begin{array}{ccc}
0 & z & 1 \\
z^{2} & 0 & 0 \\
0 & 0 & 0
\end{array}\right], \quad\left[\begin{array}{cc}
0 & z \\
z^{2} & 0
\end{array}\right]
$$

In order to reduce the system into a blocked triangular form, we have to kill $\{(3,1),(3,2)\}$ block $\left(z^{4}, z^{3}\right)$.

We have to take care which one we kill firstly $z^{4}$ or $z^{3}$ in the block. In order to examine this choice we consider

$$
\left(O\left(b_{i j}\right)-\left\{t_{i}-t_{j}+V(B)\right\}\right)_{i, j=1,2,3}=\left[\begin{array}{ccc}
1 / 2 & 0 & 0 \\
0 & 1 / 2 & 5 / 2 \\
1 & 1 / 2 & 3 / 2
\end{array}\right] .
$$

This suggests to kill $z^{3}$ on $(3,2)$-place firstly. To do so, let us consider the matrix

$$
\left[\begin{array}{cc}
0 & z \\
z^{2} & 0 \\
0 & z^{3}
\end{array}\right]
$$

A left null vector of this matrix is given by $\vec{\ell}(z)=\left(-z^{2}, 0,1\right)$. Let a transformation matrix be;

$$
\left[\begin{array}{ccc}
1 & 0 & 0 \\
0 & 1 & 0 \\
z^{2} & 0 & 1
\end{array}\right]
$$

Then the system is reduced into

$$
\left(4,\left[\begin{array}{ccc}
2 z^{2} & z & 1 \\
z^{2}+z^{5} & -z^{2} & z^{3} \\
-z^{4}+z^{5}-2 z^{6} & 0 & -z^{2}+z^{3}
\end{array}\right]\right)
$$

Next, we kill $-z^{4}$ on $(3,1)$-place. We consider the matrix

$$
\left[\begin{array}{cc}
0 & z \\
z^{2} & 0 \\
-z^{4} & 0
\end{array}\right]
$$


A left null vector of this matrix is given by $\vec{\ell}(z)=\left(0, z^{2}, 1\right)$. Let a transformation matrix be;

$$
\left[\begin{array}{ccc}
1 & 0 & 0 \\
0 & 1 & 0 \\
0 & -z^{2} & 1
\end{array}\right] .
$$

Then the system is reduced into

$$
\left(4,\left[\begin{array}{ccc}
2 z^{2} & z-z^{2} & 1 \\
z^{2}+z^{5} & -z^{2}-z^{5} & z^{3} \\
z^{5}-2 z^{6}+z^{7} & -z^{5}+2 z^{6}-z^{7} & -z^{2}+z^{3}+z^{5}
\end{array}\right]\right)
$$

By this manner we can kill $\{(3,1),(3,2)\}$ block.

In order to have a blocked diagonal form by irreducible subsystems, we have to kill $\{(1,3),(2,3)\}$ block. We, first, kill 1 on $(1,3)$-place. To do so, we consider the matrix

$$
\left[\begin{array}{ccc}
0 & z & 1 \\
z^{2} & 0 & 0
\end{array}\right] .
$$

Then a right null vector of this matrix is given by ${ }^{\mathrm{t}}(0,-1 / z, 1)$, which has a pole singularity. Let a transformation matrix be;

$$
\left[\begin{array}{ccc}
1 & 0 & 0 \\
0 & 1 & -1 / z \\
0 & 0 & 1
\end{array}\right]
$$

Then the reduced system becomes

$$
\left(4,\left[\begin{array}{ccc}
2 z^{2} & z-z^{2} & z \\
z^{2}+z^{4}+z^{5}+z^{6} & -z^{2}-z^{4}+z^{5}-z^{6} & z^{2}+z^{3}+z^{5} \\
z^{5}-2 z^{6}+z^{7} & -z^{5}+2 z^{6}-z^{7} & -z^{2}+z^{3}+z^{4}-z^{5}+z^{6}
\end{array}\right]\right)
$$

Next, in order to kill $z^{2}$ on $(2,3)$-place, let us consider a right null vector of the following matrix,

$$
\left[\begin{array}{ccc}
0 & z & 0 \\
z^{2} & 0 & z^{2}
\end{array}\right] .
$$

By using a right null vector ${ }^{\mathrm{t}}(-1,0,1)$ of this matrix, we construct a transformation matrix as above and the reduced system becomes;

$$
\left(4,\left[\begin{array}{ccc}
2 z^{2}+z^{5}+O\left[z^{6}\right] & z-z^{2}-O\left[z^{5}\right] & z-3 z^{2}+O\left[z^{3}\right] \\
z^{2}+z^{4}+O\left[z^{5}\right] & -z^{2}+O\left[z^{4}\right] & z^{3}-z^{4}+O\left[z^{5}\right] \\
z^{5}+O\left[z^{6}\right] & -z^{5}+O\left[z^{6}\right] & -z^{2}+z^{3}+O\left[z^{4}\right]
\end{array}\right]\right) .
$$


By continuing these operations we finally obtain a blocked diagonal form by irreducible subsystems,

$$
\left(4,\left[\begin{array}{cc}
O\left[z^{2}\right] & z+O\left[z^{2}\right] \\
z^{2}+O\left[z^{4}\right] & O\left[z^{2}\right]
\end{array}\right]\right) \oplus\left(4,-z^{2}+O\left[z^{4}\right]\right) .
$$

For the first irreducible subsystem, V-numbers are made flatten by a transformation matrix $\operatorname{Diag}\left[1, z^{1 / 2}\right]$, which brings to

$$
\left(4,\left[\begin{array}{cc}
O\left[z^{2}\right] & z^{3 / 2}+O\left[z^{5 / 2}\right] \\
z^{3 / 2}+O\left[z^{7 / 2}\right] & O\left[z^{2}\right]
\end{array}\right]\right) .
$$

This shows that the system is reduced into a diagonal system by a transformation matrix of formal power series of $z^{1 / 2}$.

\section{Conclusion:}

- First two terms of the determining factor of fundamental matrix solution is;

$$
\left[\begin{array}{ccc}
-1 /\left(3 z^{3}\right)+2 /\left(5 z^{5 / 2}\right) & 0 & 0 \\
0 & -1 /\left(3 z^{3}\right)-2 /\left(5 z^{5 / 2}\right) & 0 \\
0 & 0 & -1 /\left(3 z^{3}\right)+1 /\left(2 z^{2}\right)
\end{array}\right]
$$

- Newton polygon $\mathrm{N}(L)$ has two vertexes $\{(0,3),(3,12)\}$ with a side of slope 3 , which is determined from the characteristic polynomial $\operatorname{det}\left(z^{5} \lambda I_{3}-\AA_{T}(z)\right)=\left(z^{5} \lambda-z\right)^{3}$. This shows that $\chi\left(L ; \mathscr{G}^{s}\right)$ has only one discontinuous point $s=4 / 3$.

\subsection{Full rank system when the principal matrix have distinct eigenvalues}

The system we consider is

$$
L=(3, A(z)), \quad A(z)=\left[\begin{array}{cc}
z+4 z^{2} & 1+4 z \\
3 z^{2}-4 z^{3} & -z-3 z^{3}
\end{array}\right] .
$$

The Volevič weight $V(A)$ and the associated V-numbers $T=\left\{t_{i}\right\}$ are;

$$
V(A)=1, \quad T=\left\{t_{1}=0, t_{2}=1\right\} .
$$

The principal matrix $\stackrel{\circ}{A}_{T}$ and its eigenvalues $\left\{\alpha_{j}\right\}$ are;

$$
\stackrel{\circ}{A}_{T}=\left[\begin{array}{cc}
1 & 1 \\
3 & -1
\end{array}\right], \quad\left\{\alpha_{j}\right\}=\{2,-2\}
$$


A transformation matrix to make the V-numbers flat is $\operatorname{Diag}[1, z]$, and the reduced system is

$$
\left(3,\left[\begin{array}{cc}
z+4 z^{2} & z+4 z^{2} \\
3 z-4 z^{2} & -z-4 z^{3}
\end{array}\right]\right) .
$$

To the above obtained system, a transformation matrix, which brings the system into a diagonal form, is already known by [Was, Chap. IV, Sec. 12]. We follow his idea.

We, first, make the linear part of the coefficient matrix to be diagonal by the transformation matrix

$$
\left[\begin{array}{cc}
1 & 1 \\
1 & -3
\end{array}\right]
$$

Then the reduced system becomes;

$$
\left(3,\left[\begin{array}{cc}
2 z+5 z^{2}-z^{3} & -7 z^{2}+3 z^{3} \\
3 z^{2}+z^{3} & -2 z-z^{2}-3 z^{3}
\end{array}\right]\right) \underset{\text { put }}{=}(3, B(z)) .
$$

Then a transformation matrix which reduces the system into a diagonalized form is obtained by a matrix of the form,

$$
\left(\begin{array}{cc}
1 & p^{12}(z) \\
p^{21}(z) & 1
\end{array}\right), \quad p^{i j}(0)=0,
$$

where $p^{i j}(z)$ are uniquely determined from the following equations,

$$
\left\{\begin{array}{l}
b^{11}(z) p^{12}(z)-p^{12}(z) b^{22}(z)=-b^{12}(z)+z^{p+1} \frac{d}{d z} p^{12}(z)+p^{12}(z) b^{21}(z) p^{12}(z) \\
b^{22}(z) p^{21}(z)-p^{21}(z) b^{11}(z)=-b^{21}(z)+z^{p+1} \frac{d}{d z} p^{21}(z)+p^{21}(z) b^{12}(z) p^{21}(z) .
\end{array}\right.
$$

Here $b^{i j}(z)$ denotes the $(i, j)$ entry of the coefficient matrix $B(z)$.

Let $p^{21}(z)=a z+b z^{2}+c z^{3}$ be an approximate solution for the first equation. Then we get the following equations for $\{a, b, c\}$.

$$
3-4 a=0, \quad 1-6 a-4 b=0, \quad-3 a+7 a^{2}-6 b-4 c=0,
$$

which are solved by $a=3 / 4, b=-7 / 8, c=111 / 64$. That is,

$$
p^{21}(z)=\frac{3}{4} z-\frac{7}{8} z^{2}+\frac{111}{64} z^{3}+O\left[z^{4}\right] .
$$

Similarly we get

$$
p^{12}(z)=\frac{7}{4} z-\frac{27}{8} z^{2}+\frac{443}{64} z^{3}+O\left[z^{4}\right]
$$


Now an approximation of the reduced diagonal system is given by

$$
\left(3,\left[\begin{array}{cc}
2 z+5 z^{2}-25 z^{3} / 4+O[z]^{4} & O[z]^{5} \\
O[z]^{5} & -2 z-z^{2}+9 z^{3} / 4+O[z]^{4}
\end{array}\right]\right) .
$$

\section{Conclusion:}

- The determining factor of the fundamental matrix solutions is given by

$$
\operatorname{Diag}\left[-z^{-2}-5 z^{-1}, z^{-2}+z^{-1}\right] .
$$

- Newton polygon $N(L)$ has two vertexes $\{(0,2),(2,6)\}$ with a side of slope 2. This shows that $\chi\left(L ; \mathscr{G}^{s}\right)$ has only one discontinuous point $s=3 / 2$.

\section{References}

[Bal1] Balser, W., From Divergent Power Series to Analytic Functions, Lecture Notes in Math., 1582, Springer-Verlag, Berlin, 1994.

[Bal2] Balser, W., Formal Power Series and Linear Systems of Meromorphic Ordinary Differential Equations, Universitext, Springer-Verlag, New York, 2000.

[Bar] Barkatou, M. A., An algorithm to compute the exponential part of a formal fundamental matrix solution of a linear differential system, Appl. Algebra Engrg. Comm. Comput., 8 (1997), 1-23.

[B-P] Barkatou, M. and Pflügel, E., An algorithm computing the regular formal solutions of a system of linear differential equations, J. Symbolic Comput., 28 (1999), 569-587.

[Bra] Braaksma, B. L. J., Multisummability of formal power series solutions of nonlinear meromorphic differential equations, Ann. Inst. Fourier (Grenoble), 42 (1992), 517-540.

[Del] Deligne, P., Équations Différentielles à Points Singuliers Réguliers, Lecture Notes in Math., 163, Springer-Verlag, Berlin-New York, 1970.

[Hor] Horn, J., Zur Theorie der Systeme linearer Differentialgleichungen mit einer unabhängingen Veränderlichen, II, Math. Ann., 40 (1892), 527-550.

[Hs-Si] Hsieh, Po-Fang and Y. Sibuya, Basic theory of ordinary differential equations, Universitext, Springer-Verlag, New York, 1999.

[Huf] Hufford, G., On the characteristic matrix of a matrix of differential operators, J. Differential Equations, 1 (1965), 27-38.

[J-L] Jurkat, W. B. and Lutz, D. A., On the order of solutions of analytic linear differential equations, Proc. London Math. Soc. (3), 22 (1971), 465-482.

[Kit] Kitagawa, K., L'irrégularité en un point singulier d'un système d'équations différentielles linéaires d'ordre 1, J. Math. Kyoto Univ., 23 (1983), 427-440.

[Kom] Komatsu, H., On the index of ordinary differential operators, J. Fac. Sci. Univ. Tokyo Sect. IA Math., 18 (1971), 379-398.

[Lod1] Loday-Richaud, M., Solutions formelles des systèmes différentiels linéaires méromorphes et sommation, Exposition. Math., 13 (1995), 116-162.

[Lod2] Loday-Richaud, M., Rank reduction, Normal forms and Stokes matrices, Expo. Math., 19 (2001), 229-250.

[Lut] Lutz, D. A., Some characterization of system of linear differential equations having regular singular solutions, Trans. Amer. Math. Soc., 26 (1967), 424-441. 
[Mal] Malgrange, B., Sur les points singuliers des équations différentielles linéaires, Enseignement Math. (2), 20 (1974), 147-176.

[Miy1] Miyake, M., On Cauchy-Kowalevski's theorem for general systems, Publ. Res. Inst. Math. Sci., 15 (1979), 315-337.

[Miy2] Miyake, M., On the determinant of matrices of ordinary differential operators and an index theorem, Funkcial. Ekvac., 26 (1983), 155-171.

[Miy3] Miyake, M., On the irregularity for general system of differential equations in the complex domain, Funkcial. Ekvac., 26 (1983), 211-230.

[M-I] Miyake, M. and Ichinobe, K., Irregularity for singular system of ordinary differential equations in complex domain, Funkcial. Ekvac., 52 (2009), 53-82.

[M-Y1] Miyake, M. and Yoshino, M., Wiener-Hopf equation and Fredholm property of the Goursat problem in Gevrey space, Nagoya Math. J., 135 (1994), 165-196.

[M-Y2] Miyake, M. and Yoshino, M., Toeplitz operators and an index theorem for differential operators on Gevrey spaces, Funkcial. Ekvac., 38 (1995), 329-342.

[Mos] Moser, J., The order of a singularity in Fuchs' theory, Math. Z., 72 (1959/1960), 379398.

[O-K] Okubo, K. and Kohno, M., Asymptotic expansions, Kyoiku Shuppann (1976) (in Japanese).

[Pfl] Pflügel, E., Effective formal reduction of linear differential systems, Appl. Algebra Engrg, Comm. Comput., 10 (2000), 153-187.

[Ram1] Ramis, J.-P., Dévissage Gevrey, Asterisque, 59-60, Soc. Math. France, Paris, 1978, pp. 173-204.

[Ram2] Ramis, J.-P., Théorèmes d'indices Gevrey pour les équations différentielles ordinaires, Mem. Amer. Math. Soc., 48 (1984), no. 296, 95pp.

[Ram3] Ramis, J.-P., Les séries $k$-summable et leurs applications, Lecture Notes in Phys., 126, Springer-Verlag, Berlin-New York, 1980, pp. 178-199.

[R-S] Ramis, J.-P. and Sibuya, Y., A new proof of multisummability of formal solutions of non linear meromorphic differential equations, Ann. Inst. Fourier (Grenoble), 44 (1994), 811-848.

[Sib] Sibuya, Y., Ordinary differential equations in complex domain, Kinokuniya (1976) (in Japanese); Linear Differential Equations in the Complex domain: Problem of Analytic Continuation, Translations of Mathematical Monographs, 82, American Mathematical Society, Providence, RI, 1990.

[Vol] Volevič, L. R., On general system of differential equations, Dokl. Acad. Nauk SSSR, 132 (1960), 20-23; Soviet Math. Dokl., 1 (1960), 458-461.

[Was] Wasow, W., Asymptotic Expansions for Ordinary Differential Equations, Pure and Applied Mathematics, Vol. XIV, Interscience Publishers John Wiley \& Sons, Inc., New York-London-Sydney, 1965 (Also Dover, New York, 1987).

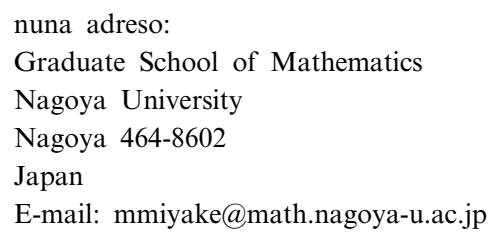

(Ricevita la 15-an de marto, 2011)

(Reviziita la 30-an de julio, 2011) 